\title{
Touching upon regulators of Piezo2 in mouse somatosensation
}

\author{
Dissertation \\ for the award of the degree \\ "Doctor rerum naturalium" (Dr.rer.nat.) \\ of the Georg-August-Universität Göttingen
}

within the International Max Planck Research School (IMPRS), Neuroscience

of the Georg-August University School of Science (GAUSS)

submitted by

\section{Pratibha Narayanan}

from New Delhi

Göttingen, 2017 


\section{Thesis Committee}

Dr. Manuela Schmidt

Prof. Dr. Martin Göpfert

Prof. Dr. Luis A. Pardo

\section{Members of the Examination Board}

Dr. Manuela Schmidt

Prof. Dr. Martin Göpfert
Somatosensory signaling and Systems Biology group, Max Planck Institute for Experimental Medicine

Dept. of Cellular Neurobiology, SchwannSchleiden Research Centre

Dept. of Molecular Biology of Neuronal Signals, Max Planck Institute for Experimental Medicine

\section{Further members of the Examination Board}

Prof. Dr. Luis A. Pardo

Prof. Dr. Tobias Moser

Prof. Dr. Silvio O. Rizzoli

Prof. Dr. Michael Sereda
Somatosensory signaling and Systems Biology group, Max Planck Institute for Experimental Medicine

Dept. of Cellular Neurobiology, SchwannSchleiden Research Centre

Dept. of Molecular Biology of Neuronal Signals, Max Planck Institute of Experimental Medicine

Institute for Auditory Neuroscience \& InnerEarLab

Dept. of Neuro-and Sensory Physiology, University Medical Center

Molecular and Translational Neurology group, Max Planck Institute for Experimental Medicine 


\section{TABLE OF CONTENTS}

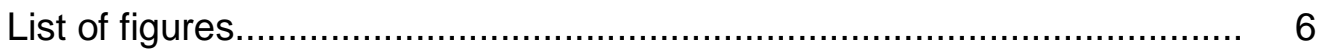

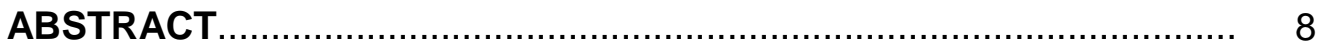

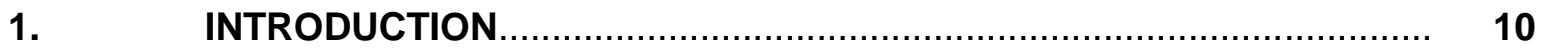

1.1. Somatosensation and mechanotransduction...................................... 10

1.1.1. Somatosensory systems.......................................................... 10

1.1.2. Mechanotransduction................................................................... 11

1.1.3. Ion channels in mechanotransduction................................................ 12

1.1.4. Sensory mechanotransduction........................................................... 13

1.2. Piezo2 protein family................................................................... 14

1.2.1. Structure of Piezo proteins................................................................ 15

1.2.2. Piezo2 in somatosensory mechanotransduction..................................... 16

1.2.3. Pathology associated with Piezo2 ..................................................... 18

1.3. Protein complexes associated with ion channels...................... 19

1.3.1. Ion channel function and protein complexes.......................... 19

1.3.2. Protein complexes in mechanotransduction.......................... 19

1.3.3. Protein complexes in mammalian somatosensation...................... 20

1.3.4. Piezo2 associated proteins (Piezo2 interactors) ........................ 21

1.4. Potential Piezo2 interactors: Pericentrin and Mtmr2 .................... 24

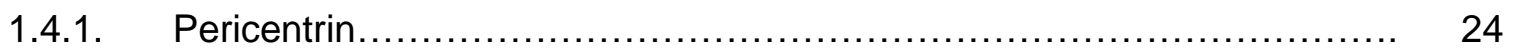

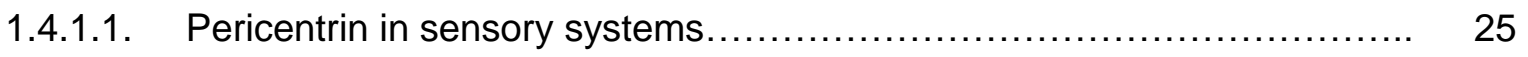

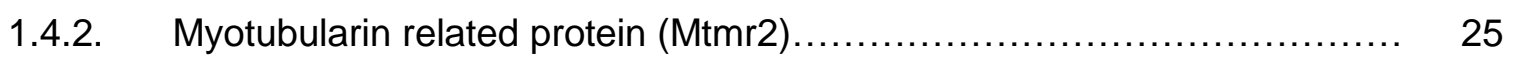

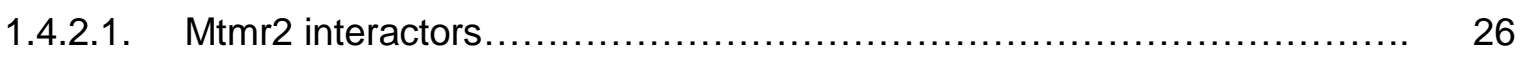

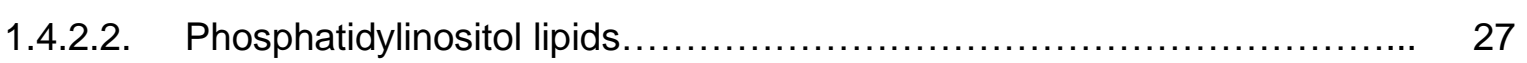

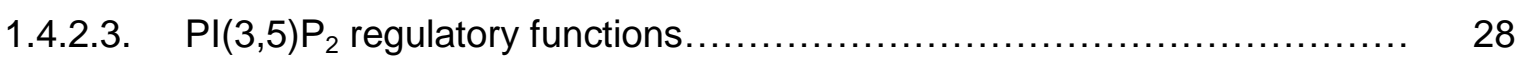

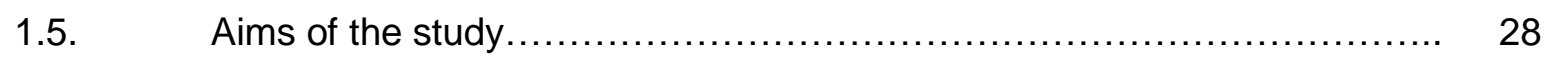

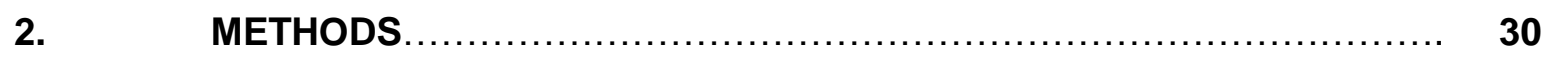

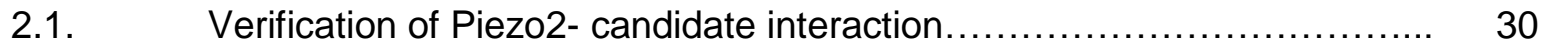

2.1.1. Transient transfection of HEK293 cells............................. 30

2.1.2. Co-immunoprecipitation in HEK293 cells............................... 30

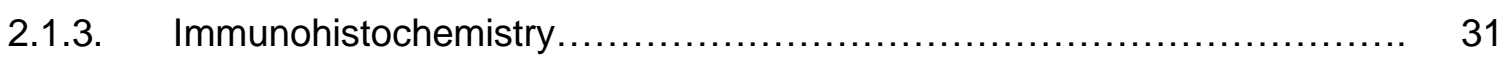

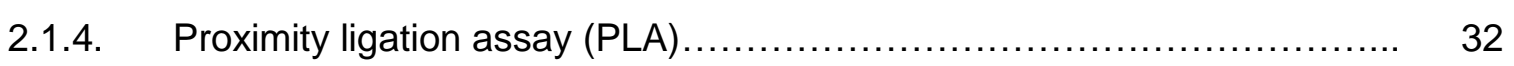

2.2. Functional characterization of interactors............................. 33

2.2.1. DRG cultures and transfection............................................ 33

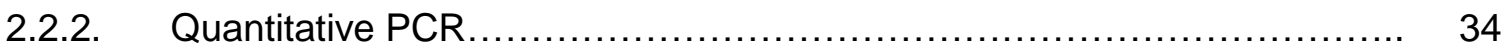

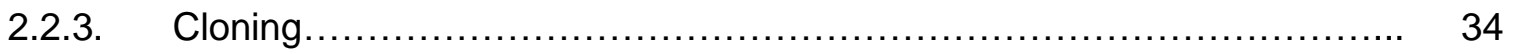

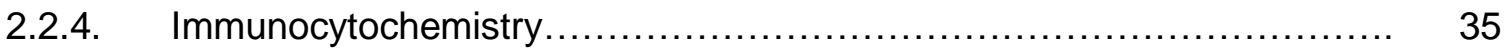




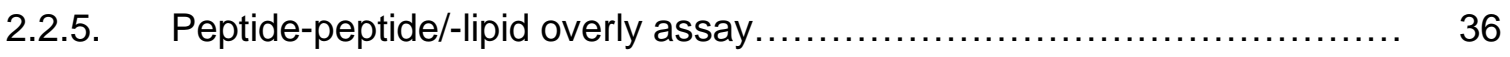

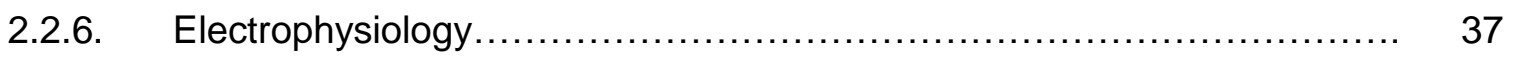

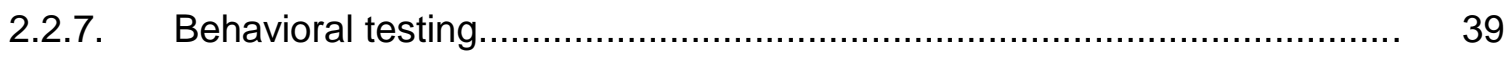

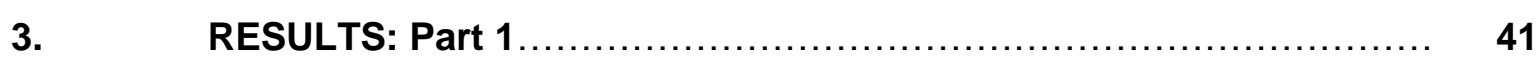

3.1. Verification of Piezo2-Pericentrin interaction.............................. 41

3.1.1. Piezo2-Pcnt co-immunoprecipitate in HEK293 cells....................... 41

3.1.2. Pcnt is expressed in somatosensory system ........................... 43

3.2. Functional characterization of Piezo2-Pcnt interaction..................... 46

3.2.1. Loss of Pcnt potentiates Piezo2 currents................................ 48

3.2.2. Loss of Pcnt does not affect other MA currents....................... 50

3.2.3. Pcnt overexpression does not influence Piezo2 currents................... 52

3.3. Mechanism of Piezo2-Pcnt interaction................................... 53

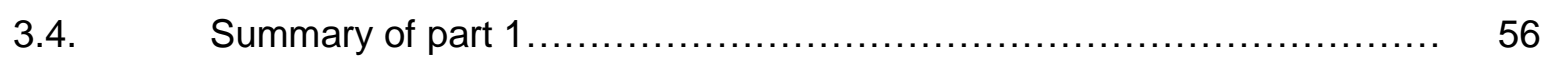

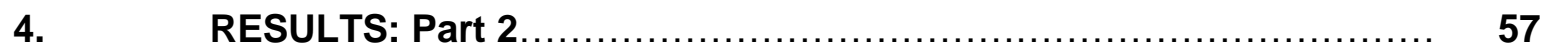

4.1. Verification of Piezo2-Mtmr2 interaction............................... 57

4.2. Characterization of Piezo2-Mtmr2 interaction in HEK293 cells............. 59

4.2.1. Mtmr2 co-expression suppresses Piezo2 currents........................ 59

4.2.2. Piezo1 and Kv1.1 currents are not affected............................ 61

4.3. Characterization of Piezo2-Mtmr2 in DRG neurons ...................... 62

4.3.1. Mtmr2 co-expression suppresses Piezo2 currents..................... 62

4.3.2. Mtmr2 siRNA does not affect Piezo2 expression........................... 64

4.3.3 Mtmr2 knockdown potentiates Piezo2 currents........................... 65

4.3.4. MA populations are re-distributed upon Mtmr2 siRNA ................... 67

4.4. Mechanism of Piezo2-Mtmr2 interaction ............................. 69

4.4.1. Catalytic activity of Mtmr2 is essential to suppress RA-MA currents........ 69

4.4.2. Apilimod application reverses Mtmr2 knockdown phenotype............... 71

4.4.3. Artificial PIPs do not affect RA-MA currents............................. 74

4.4.4. Piezo2 and Mtmr2 contain $\mathrm{PI}(3,5) \mathrm{P}_{2}$ binding motifs..................... 76

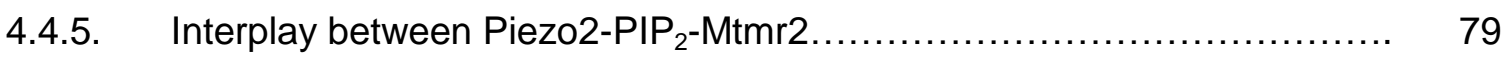

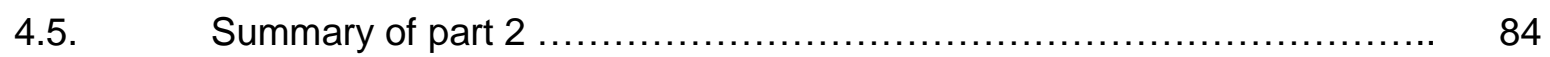

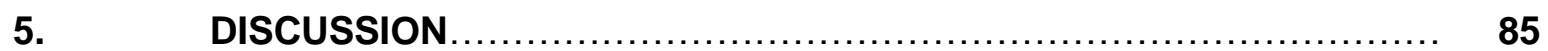

5.1. Relevance of studying Piezo2 protein complexes....................... 85

5.1.1. Biological significance of identified candidates........................... 86

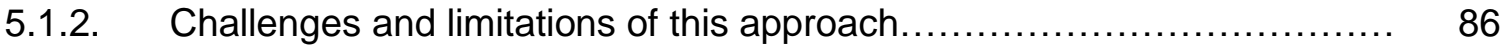

5.2. Identification of Pericentrin as Piezo2 interactor........................ 87

5.3. Identification of Mtmr2 as a Piezo2 interactor......................... 89

5.4. Piezo2 function is controlled by a defined set of interacting proteins....... 94 
6.

REFERENCES.

ACKNOWLEDGEMENTS .......................................................... 107

Supplementary tables............................................. 108

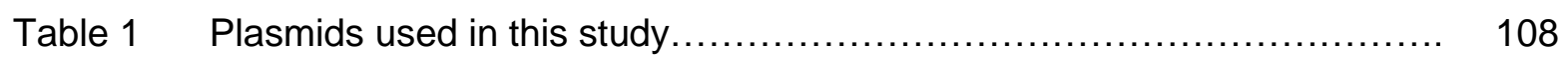

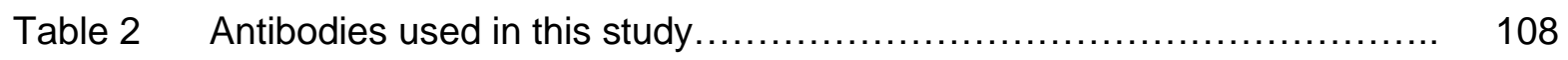

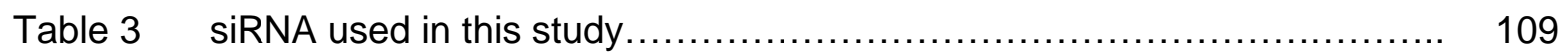

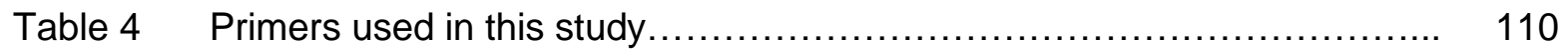

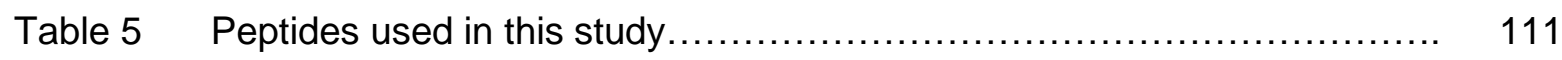

Table $6 \quad$ Reagents used in this study ..................................... 111

Table $7 \quad$ List of abbreviations.................................................................... 114

DECLARATION.................................................... 116

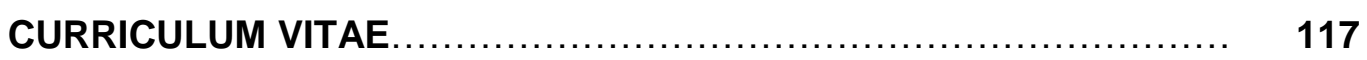




\section{LIST OF FIGURES}

Figure 1 Schematic illustration of mammalian somatosensory pathway........... 11

Figure 2 Structure of Piezo2 proteins.......................................................... 16

Figure $3 \quad$ Illustrations of mechanosensitive channel complexes.................. 20

Figure 4 Identified components of mammalian somatosensory complex........... 22

Figure 5 Scheme of MS based interactomics approach to identify Piezo2

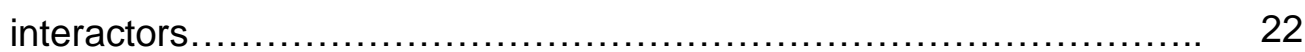

Figure 6 Quantitative MS based interactomics approach to identify Piezo2

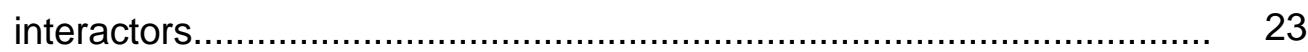

Figure $7 \quad$ Pericentrin structure and isoforms.................................................... 24

Figure $8 \quad$ Mtmr2 structure and phospholipid catalysis pathway..................... 26

Figure $9 \quad$ Illustration depicting recording of MA currents........................... 37

Figure 10 Co-IP of Piezo2 and Pericentrin in HEK293 cells..................... 42

Figure 11 Pericentrin expression in mammalian somatosensory system............ 44

Figure 12 Piezo2 and Pericentrin express in close proximity in DRG neurons...... 46

Figure 13 Knockdown of Pericentrin in DRG neurons.......................... 47

Figure 14 Pericentrin knockdown in DRG neurons potentiates RA-MA currents.... 49

Figure 15 Pericentrin knockdown does not affect IA-MA and SA-MA currents...... 51

Figure 16 Pericentrin overexpression in DRG neurons and HEK293 cells.......... 53

Figure 17 Exploring mechanisms of Piezo2-Pericentrin interaction.................. 54

Figure 18 Mtmr2 expression in mouse DRG neurons............................ 57

Figure 19 Piezo2 and Mtmr2 are expressed in close proximity in HEK293 cells.... 58

Figure 20 Mtmr2 co-expression with Piezo2 in HEK293 cells suppresses RA-MA

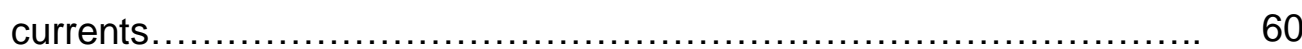

Figure 21 Mtmr2 co-expression does not affect Piezo1 or Kv1.1 currents in HEK293 cells.......................................................... 61

Figure 22 Mtmr2 overexpression in DRG neurons suppresses RA-MA currents.... 63

Figure 23 Knockdown of Mtmr2 in DRG neurons.............................. 64

Figure 24 Mtmr2 knockdown in DRG neurons potentiates RA-MA currents........ 66

Figure 25 Mtmr2 knockdown does not modulate IA-MA and SA-MA currents...... 68

Figure 26 Mtmr2C417S does not affect Piezo2 currents in HEK293 cells........... 70

Figure 27 Mtmr2 knockdown can be rescued by Apilimod application............... 74

Figure 28 Synthetic PIP addition to DRG neurons does not affect RA-MA

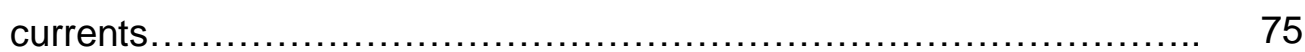

Figure $29 \mathrm{PIP}_{2}$ binding sequences in Piezo2, Mtmr2 and TRPML1 ................ 77

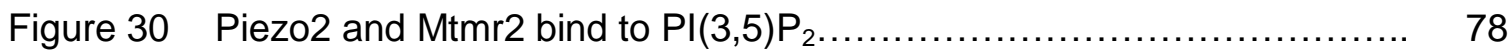


Figure 31 Apilimod rescues potentiation of RA-MA currents and mechanical

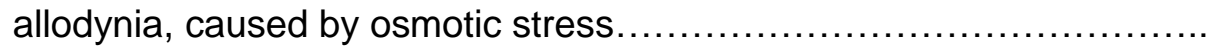

Figure 32 Hypotonic intracellular stress rescues RA-MA potentiation upon loss of Mtmr2 ................................................................. 83

Figure 33 Illustration of Piezo2-PI(3,5) $\mathrm{P}_{2}-\mathrm{Mtmr2}$ interaction...................... 93 


\section{ABSTRACT}

Mechanosensation is an integral property of sensory systems like audition and somatosensation. The molecular determinants of mechanotransduction in mammals are only beginning to be understood. In 2010, Piezo2, a member of the Piezo family, was identified as the bona fide mechanotransducer in the somatosensory system of mice. Since then Piezo2 has been implicated in light touch, proprioception, mechanical allodynia and hyperalgesia. In order to understand more about the function of Piezo channels and their role in mechanosensation, an understanding of its regulators is vital. Protein-protein interactions are the bedrock of ion channel regulation and in the context of Piezo2, few such interactions (Stoml3 and Runx1) have been described. Recently it has also been found that the activity of Piezo2 is linked strongly to the properties of the cellular membrane. Depletion of cholesterol, mediated by Piezo2 interactor Stoml3, was shown to potentiate Piezo2 mediated currents. Furthermore, TRPV1 mediated modulation of Piezo2 was shown to involve membrane phospholipid $\mathrm{PI}(4,5) \mathrm{P}_{2}$.

In 2016, our lab reported the first systematic characterization of the Piezo2 interactors in somatosensory neurons of mice. Through a mass-spectrometry based interactomics approach, 36 proteins were identified as potential Piezo2 interactors. Within these proteins we found some known modulators of somatosensory mechanotransduction including Kv1.1 and CamKII. Besides these, there were some proteins which presented as highly unlikely interactors of Piezo2, such as Pericentrin (Pcnt). Other proteins identified in the screen were involved in cellular processes like protein localization, protein phosphorylation and vesicle mediated transport.

In the current study, I describe the characterization of two of the Piezo2 interactors identified in the mass-spectrometry screen. The first part of the results describes Pericentrin (Pcnt) as a low probability interactor of Piezo2. The results show that Pcnt is expressed in close proximity with Piezo2 in both peripheral endings and cell bodies of DRG neurons. Additionally, Pcnt is shown to functionally modulate Piezo2 in DRG neurons. Loss of Pcnt in these neurons was found to potentiate Piezo2 currents and increase Piezo2 membrane expression. Though the mechanism of Piezo2-Pcnt interaction remains to be explored, the results do provide validation of the interactomics screen. The fact that the screen can successfully identify bona fide Piezo2 interactors makes it a useful resource for understanding Piezo2 function.

The second part of the results aims to characterize the interaction between Piezo2 and Mtmr2. Mtmr2, is a member of the myotubularin phosphatase family which is involved in the phospholipid synthesis pathway. It was identified as a high probability interactor of 
Piezo2, and notably, two other members of the same family, Mtmr1 and Mtmr5 (Sbf2) were also identified in the screen. The results presented here suggest that Mtmr2 is involved in a bi-directional regulation of Piezo2 function in DRG neurons i.e. loss of Mtmr2 potentiates Piezo2 mediated currents and overexpression of Mtmr2 suppresses Piezo2 currents. Furthermore, the phosphatase activity of Mtmr2 was found to be indispensable for its regulation of Piezo2. Pharmacological modulations of the phospholipid pathway show that $\mathrm{PI}(3,5) \mathrm{P}_{2}$ plays a crucial role in the regulation of Piezo2. Further evidence for phospholipid interaction with Piezo2 came with the identification of a peptide segment of Piezo2 that can bind to $\mathrm{PI}(3,5) \mathrm{P}_{2}$. Additionally, modulation of Piezo2 by osmotic stress is shown to rely on $\mathrm{PI}(3,5) \mathrm{P}_{2}$ levels and Mtmr2 activity. The results presented here provide additional evidence for regulation of Piezo2 by membrane lipids and how proteins regulating these lipids are relevant to Piezo2 physiology.

In summary, since the identification of Piezo2, a quest to learn about its function and regulation has been underway. The results of this study combined with the interactomics screen published in 2016 have attempted to answer some fundamental questions about Piezo2 physiology. A characterization of the other proteins identified in our screen will help reveal more facets of Piezo2 regulation. Future studies will also add to structural information on Piezo2 which can further help elucidate its role as the bona fide mechanotransducer of the mammalian somatosensory system. 


\section{INTRODUCTION}

\subsection{Somatosensation and mechanotransduction}

\subsubsection{Somatosensory system}

All organisms are constantly exposed to a wide range of stimuli, the processing of which is crucial to their behavior. Sensory systems have evolved to detect and process these different stimuli thus allowing for a functional representation of the world. The somatosensory system has evolved to detect mechanical, chemical and thermal noxious and innocuous cues from the external environment and from within the body. What is remarkable is the ability of this system to process a wide range of stimuli from the stroking of a mild feather to a strong and unpleasant hit from a hammer. Unlike other sensory systems, somatosensation lacks a defined sensory organ but rather receives its information from skin, viscera and other sensory organs including the tongue, nose etc. In vertebrates, the cell bodies of somatosensory neurons reside in the dorsal root ganglia (DRGs) and the trigeminal ganglia (TGs). These neurons are pseudo unipolar which project one axonal branch to the periphery (for example to the skin) and the other branch to the dorsal horn of the spinal cord where they synapse with second order neurons. Because of the importance of injury and immediate repair to an organism's survival, a large proportion of DRG neurons are tuned to detect noxious stimuli. These constitute the high threshold mechanoreceptors (HTMRs), while innocuous mechanical stimuli are detected by the low threshold mechanoreceptors (LTMRs). The nerve fibers belonging to these mechanoreceptors can be classified as $A \beta, A \delta$ and C-fibers, based on their conduction velocities. At the level of the spinal cord, information travels through two pathways; innocuous stimulus information travels through the dorsal column medial lemniscus pathway up to the medial lemniscus where it decussates to the contralateral side and synapses in the thalamus. The noxious stimulus on the other hand decussates to the contralateral side at the spinal cord level and goes through the spino-thalamic track/anterolateral tract also synapsing in the thalamus. All the information culminates in the primary somatosensory cortex. This information may integrate with signals from the other brain areas to generate the final percept (Figure 1) (Abraira and Ginty, 2013; Chalfie, 2009; Kandel et al., 2000; Zhao et al., 2016). 


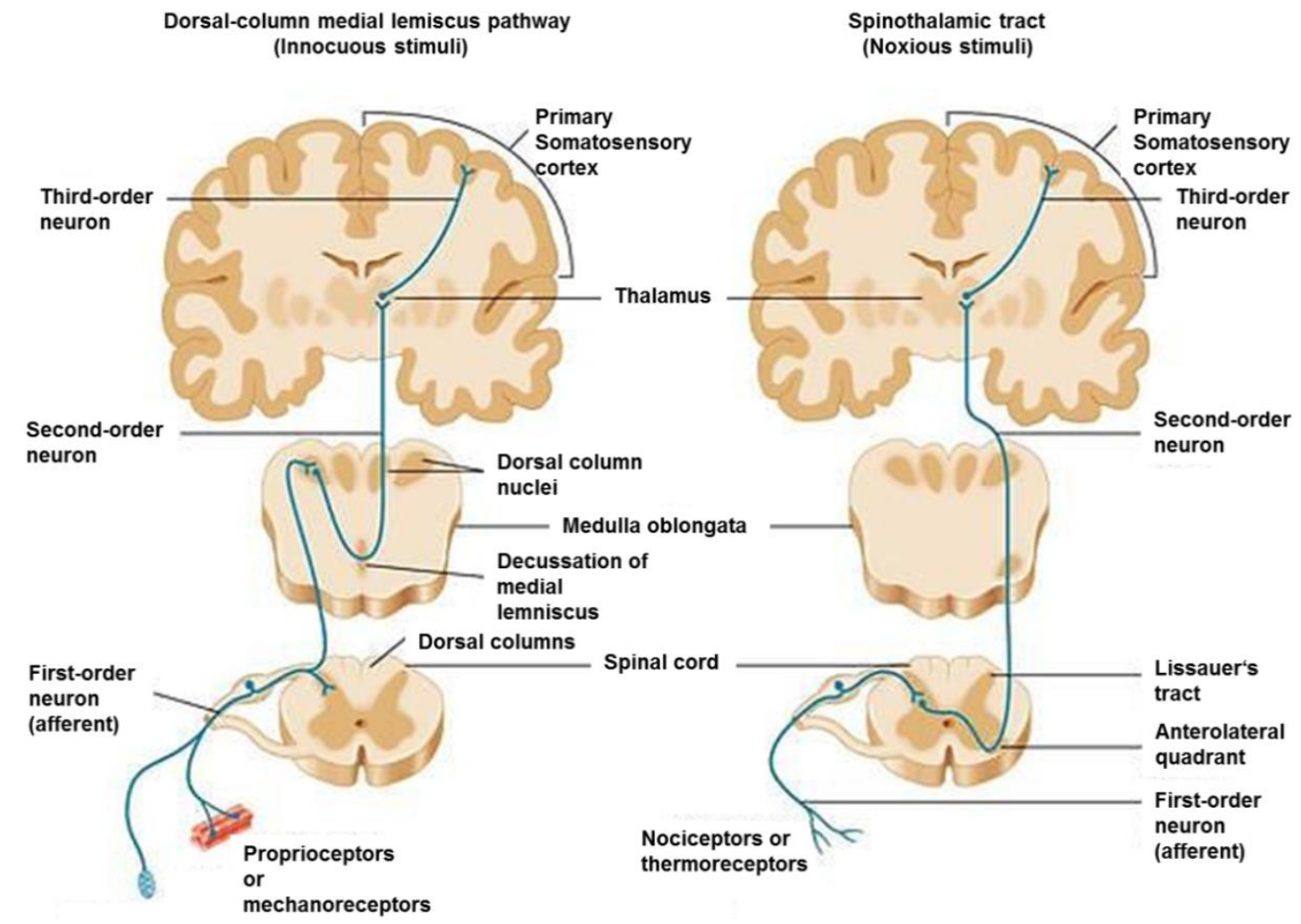

Figure 1: Schematic illustration of mammalian somatosensory pathway

The dorsal-column medial lemniscus pathway (left) carries information of innocuous mechanical stimuli while the spinothalamic tract (right) carries nociceptive or noxious stimulus information. Both tracts synapse in the thalamus and project to the primary somatosensory cortex. Adapted from Kandel et al. (2000).

\subsubsection{Mechanotransduction}

Mechanotransduction is the process of converting mechanical stimuli to biologically relevant signals. It is a fundamental requirement for many biological processes including but not limited to respiration, kidney function, circulation and somatosensation (Delmas et al., 2011). Earliest evidence of mechanosensation in evolution can already be seen in archaea and eukarya, highlighting the importance of this process. In these early organisms, mechanotransduction played a role in cell protection and survival. As we look through evolution, mechanotransduction is a defining property of touch receptors in worms, bristle receptors in flies and auditory and skin mechanoreceptors in vertebrates (Delmas et al., 2011; Gillespie and Walker, 2001). But in addition to these specialized sensory cells, every cell may be capable of sensing mechanical stress through changes at single protein level or conformational changes in protein complexes. These mechanosensitive complexes are essential for the cells to incorporate information about shear stress, tension and compression and respond appropriately to these cues (Delmas and Coste, 2013). In the context of vertebrate sensory mechanotransduction, the mechanoreceptor can respond to touch or the high frequency deflections of hair cells. An 
essential feature of this neurosensory mechanotransduction is speed. This extremely rapid mechanotransduction has brought forth the notion that the mechanosensory transduction in neuronal systems must be mediated by ion channels or ion channel complexes (Chalfie, 2009; Ernstrom and Chalfie, 2002).

\subsubsection{Ion channels in mechanotransduction}

The first discovery of ion channels that can be activated by mechanical forces was made in embryonic chick skeletal muscle and frog muscle (Brehm et al., 1984; Guharay and Sachs, 1984). For an ion channel to be classified as a bona fide mechanically activated (MA) channel it must fulfil certain criteria; firstly, it must be expressed in the mechanosensory organ to facilitate its sensory function. Secondly, the channel must be inherently mechanosensitive and contain the pore-forming subunit within itself. Thirdly, loss of the channel in the sensory cell should abolish the mechanosensitive property of the cell. This condition is necessary (but not sufficient) to prove that an ion channel is the bona fide mechanotransducer. And fourthly, the channel should retain its mechanosensitivity when expressed in heterologous systems or lipid bilayers (Arnadóttir and Chalfie, 2010; Ranade et al., 2015). The bacterial mechanosensitive channels provide a good example of fulfilling these criteria. Mechanically gated channels in bacteria respond to hypo-osmotic shock/stress conditions by allowing the release of solutes. Two of these channels have been shown to be bona fide mechano-gated channels; Mechanosensitive channel of large conductance (MscL) and Mechanosensitive channel of small conductance (MscS). Loss of these channels (only both together) causes loss of response to osmotic shock in bacteria. Point mutations in the genes encoding these channels can alter the osmotic responses of the bacteria, and lastly and most importantly, these channels when reconstituted in liposomes can be activated by pressure without the need for additional components. Taken together, this illustrates that MscL and MscS are the bona fide mechanically gated channels in bacteria (Arnadóttir and Chalfie, 2010). Besides the above mentioned bacterial channels, few candidates have been discovered that meet all the criteria described above. In the next section, I discuss mechanotransduction in the context of sensory systems and more specifically the somatosensory system. 


\subsubsection{Sensory mechanotransduction}

As mentioned earlier, mechanotransduction is a requirement for many physiological functions such as the pulmonary, vascular and cardiovascular systems (Ranade et al., 2015). But in this study, I focus on the role of mechanotransduction in the sensory systems. The study of mechanically gated channels in sensory systems has been confounded with technical difficulties which make progress slow. Firstly, the sparsity of the ion channel receptors in sensory cells or peripheral receptors makes biochemical characterization fairly difficult. Secondly, the absolute numbers of these channels are low, for example only 50-100 copies of the transduction channel are thought to be expressed in each vertebrate hair cell. And lastly, a key criteria for bona fide channels; functional expression in heterologous systems has proved to be a challenge (Chalfie, 2009). Despite these difficulties, to date four classes of ion channels have been identified that satisfy the above mentioned requirements for bona fide mechanosensitive channels in eukaryotes; DEG/ENaC, TRPN, TMCs and Piezos (Delmas and Coste, 2013).

The DEG/ENaC (degenerin/Epithelial Sodium Channel, Ranade et al. (2015)) channels are expressed in the touch receptor neurons of $C$. elegans where they can be mutated to produce touch insensitive mutants (Chalfie, 2009). The mechanosensitivity in these neurons is conferred by a protein complex consisting of MEC-4 and MEC-10 (DEG/ENaC proteins that are the pore forming subunits of the complex) and MEC-2 and MEC-6 which regulate the complex (Arnadóttir and Chalfie, 2010). Among the TRP (transient receptor potential), channels various members have shown potential mechanosensitivity. One particular member NompC (TRPN) was shown to be the mechanotransduction channel for gentle touch in Drosophila (Yan et al., 2013). A loss of NompC (no mechano-receptor potential), as the name suggests, leads to loss of mechanically induced receptor potential in the bristle mechanosensors of the fly (Chalfie, 2009). Additionally, two Drosophila TRPV genes, Nanchung and Inactive, and TRPA1 ortholog, Painless are necessary for touch and hearing in flies, while the TRPA1 ortholog in C.elegans is involved in somatosensation (Ranade et al., 2015). In mammals, TRPA1 was shown to be expressed in small diameter DRG neurons and mediate slowly adapting mechanically activated (SA-MA) currents in C-fiber type (small diameter) sensory neurons (Vilceanu and Stucky, 2010). The next class of bona fide MA channels was described in the context of mammalian hearing. For a long time the auditory mechanotransducing channel remained elusive and various known ion channel families including TRP channels were implicated (Ranade et al., 2015). In 2011, the TMC1 and TMC2 (Transmembrane channel 1 and 2) were shown to be required for hair cell mechanotransduction (Delmas and Coste, 2013). Besides these channels, two more components, TMHS (tetra-span membrane protein of hair cell stereocilia) and TMIE (transmembrane inner ear expressed 
gene) were shown to be necessary components of the transducing apparatus (Ranade et al., 2015).

The breakthrough in mammalian somatosensation happened in 2010. Using an extensive siRNA screening Coste and colleagues, identified Piezo1 and Piezo2 as being bona fide mechanosensitive ion channels (Coste et al., 2010; Coste et al., 2012).

\subsection{Piezo protein family}

Piezo proteins are multi-pass transmembrane proteins with very little similarity to any known protein family. A single Piezo protein has been found in many animal, plant and eukaryotic species. Vertebrates express two members of the Piezo family i.e. Piezo1 (Fam38A) and Piezo2 (Fam38B). Piezo1 is strongly expressed in bladder, colon, kidney, lung, bone, red blood cells and skin (Coste et al., 2010; Wu et al., 2016b). It shows relatively low mRNA expression in DRG (dorsal root ganglia; which contain cell bodies of somatosensory neurons). Piezo2 on the other hand shows strong expression in the DRG sensory neurons, which points to its role in somatosensory mechanotransduction. Besides DRG neurons, Piezo2 is also expressed in Merkel cells in the skin, chondrocytes in the bone, bladder, colon and lung (Coste et al., 2010; Wu et al., 2016b). Piezo protein function assayed in DRG neurons, N2A cells and HEK293 cells showed that both Piezo1 and Piezo2 generate MA currents. These currents are characterized by a rapid activation and inactivation. In a subsequent study, it was shown that Piezos are indeed poreforming ion channels that mediate MA currents. Piezos are non-selective cation channels with a mild $\mathrm{Ca}^{2+}$ preference. In near physiological conditions single-channel conductance of mouse Piezo1 (mPiezo1) is approximately 29pS and approximately 24pS for mouse Piezo2 (mPiezo2) (Coste et al., 2012; Wu et al., 2016b). Several pharmacological agents have been shown to modulate Piezo channels. Ruthenium Red and Gadolinium, known blockers of many cationic MA currents also block Piezo1 and 2 in a reversible manner, in C2C12 cells (Coste et al., 2010). Another molecule, FM1-43, a styryl dye was shown to permeate mechanosensitive ion channels in DRG neurons and block all MA currents from these cells including Piezo2-mediated currents (Drew and Wood, 2007; Eijkelkamp et al., 2013). Additionally, GsMTx4 (peptide toxin from Grammostola spatulata spider venom) non-specifically blocks cation-selective stretch sensitive channels including Piezo1 and Piezo2 (Alcaino et al., 2017; Bagriantsev et al., 2014). Besides these nonspecific inhibitors of Piezo channels, recently, Yoda1 was described as a chemical activator of Piezo1 (Piezo2 is resistant to Yoda1). Yoda1 was capable of sensitizing mPiezo1 activation and slowing the inactivation phase of the MA current. In cell-attached patch configuration of Piezo1 over expressing HEK293 cells, Yoda1 application was also 
capable of generating small currents in the absence of mechanical stimulus (Syeda et al., 2015).

\subsubsection{Structure of Piezo proteins}

A distinct feature of Piezo channels is their large size; mPiezo1 contains 2547 amino acids while mPiezo2 contains 2822 amino acids (Soattin et al., 2016). These proteins are not similar to any known proteins or ion channels. Within the Piezo family, mPiezo1 and mPiezo2 show 39\% sequence similarity (based on NCBI BlastP tool; Soattin et al. (2016). Although the details of Piezo structure remain to be unambiguously characterized, they have been proposed to contain over 18 transmembrane domains making them one of the largest ion channel complexes identified so far (Figure 2A; Ranade et al. (2015)). The first study to identify structural motifs in Piezo channels was conducted by Kamajaya and colleagues in 2014. They found that the C-terminal loop 2 (CTL2) domain forms a previously uncharacterized $\beta$-sandwich fold structure. This domain was identified in the C.elegans Piezo structure, and found to be relatively conserved across homologs. The authors postulated a role for this domain either in trafficking, tethering or ligand binding (Kamajaya et al., 2014). Subsequent studies on Piezo1 structure, found the C-terminal region (residues 1974 to C-term) essential for its ion permeation properties. Further, it was also identified that a conserved amino acid residue (E2133 in mPiezo1) which is in the vicinity of the ion permeation domain can alter the pore-dependent properties of the channel (Coste et al., 2015). In 2016, two groups identified more details of the Piezo1 structure using cryo-electron microscopy (cryo-EM) and structure-guided functional studies (Xu, 2016). These studies revealed that Piezo1 consists of peripheral transmembrane domains which assemble as a propeller with three curved 'blades'. This makes up the mechanosensing domain of the channel. In addition the two transmembrane segments in the C-terminal region encase the channel pore topped by a cap-like C-terminal extracellular domain (CED) (Figure 2B and 2C; Ge et al. (2015), Wu et al. (2016b) and Zhao et al. (2016)). The latest study exploring Piezo1 structure used magnetic nanoparticles to apply localized mechanical forces combined with pressureclamp electrophysiology to identify two mechanosensitive domains in the Piezo1 structure (Wu et al., 2016a). In the case of Piezo2 channels, structural studies have not been conducted so far and it remains to be seen if similar gating mechanisms operate in Piezo2. Though, the similarities in the two proteins in their size and predicted topology as well as the $54 \%$ sequence similarity in the C-terminal pore domain (transmembrane segments and C-terminal extracellular domain) could be indicative of similar architecture (Wu et al., 2016b). 
A

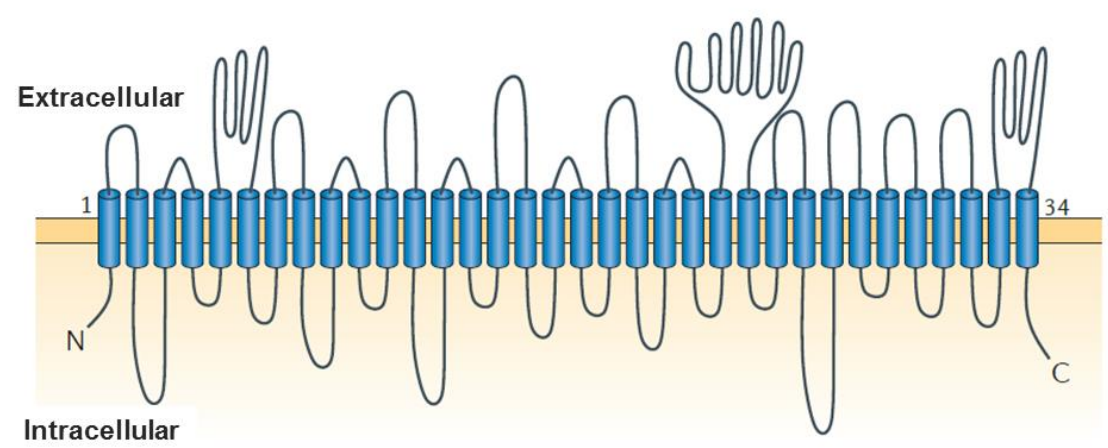

B

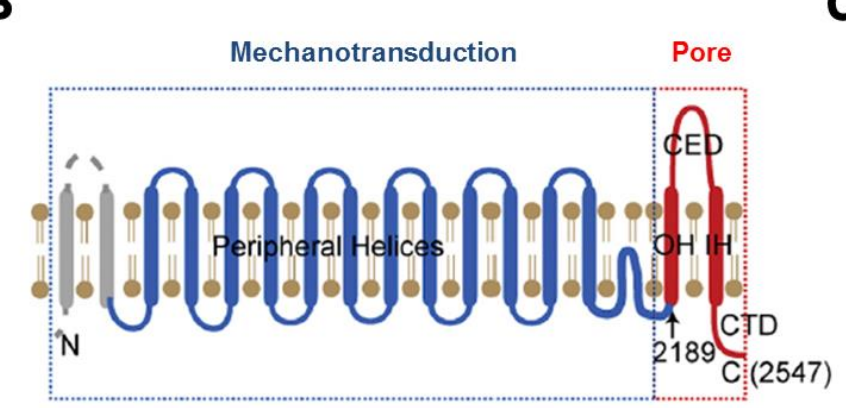

C

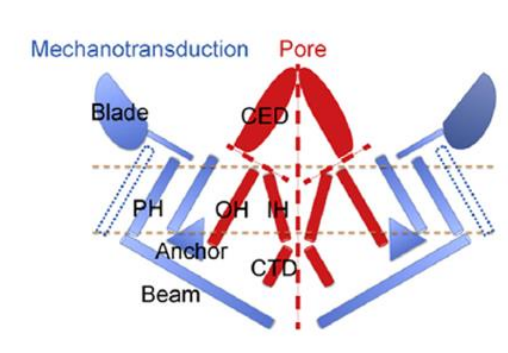

Figure 2: Structure of Piezo proteins

(A) Predicted topological structure of mPiezo2 as obtained using the transmembrane hidden Markov model (TMHMM). The structure of mPiezo2 remains to be resolved and this model only serves as a prediction for the possible structure of the channel. (B) Illustration of the topological model and (C) structural model for mPiezo1 as determined by cryo-electron microscopy shows two distinct domains, namely the peripheral domain (peripheral helices; $\mathrm{PH}$ ) which assembles into the mechanotransducing propeller 'blade' structure and the C-terminus pore region capped by the C-terminal extracellular domain (CED). CTD: C-terminal domain; OH: Outer helix; IH: Inner helix. Adapted from Delmas and Coste (2013) and Zhao et al. (2016)

\subsubsection{Piezo2 in somatosensory mechanotransduction}

As mentioned earlier the Piezo proteins are expressed in variety of tissues and cell types. Piezo1 shows a largely non-sensory distribution while Piezo2 is highly enriched in the DRGs. The DRG neurons can be anatomically categorized into large, medium and small diameter neurons wherein the large diameter neurons are thought to be mechanoreceptor neurons while the medium and small diameter neurons are considered nociceptor neurons (Julius and Basbaum, 2001). These different populations express marker proteins such as Peripherin, Neurofilament 200 (NF200) etc. Piezo2 is expressed in a subset of DRG neurons expressing NF200 and Peripherin (markers of mechanosensory neurons). A small percentage of overlap was also observed with TRPV1 (marker of nociceptive neurons) (Coste et al., 2010). Further, DRG neurons can be classified functionally based on the type of MA current they exhibit. One can identify three types of MA currents based on their inactivation time constant $(\tau) . \tau<10 \mathrm{~ms}$ are referred to as Rapidly adapting (RA) currents; $10 \mathrm{~ms}<\tau<30 \mathrm{~ms}$ are the Intermediately 
adapting (IA) currents; and $\tau>30 \mathrm{~ms}$ are the Slowly adapting (SA) currents (Coste et al., 2010). It was shown that upon siRNA mediated knockdown of mPiezo2 from primary cultures of DRG neurons, the proportion of neurons showing RA-MA currents was significantly reduced and the neurons not responding to mechanical stimulus were concomitantly increased. The fact that the other MA current populations remain unaffected showed that Piezo2 specifically mediates RA-MA currents in these neurons (Coste et al., 2010). At the periphery, somatosensory neurons can be divided into two broad categories; High-threshold mechanoreceptors (HTMRs) and low-threshold mechanoreceptors (LTMRs). The HTMRs respond to noxious range of mechanical somatosensory stimuli while the LTMRs respond to innocuous/benign stimuli. The nerve fibers associated with these receptors are $A \beta$ fibers, $A \delta$ and $C$-fibers. The $A \beta$ fibers have low mechanical thresholds and are therefore suited to be detectors of light touch. The majority of $A \delta$ and $\mathrm{C}$-fibers are believed to be nociceptors/detectors of noxious stimuli. Though, there are subsets of the $A \delta$ and $C$-fibers that have also been implicated in light touch due to their considerably lower thresholds. These fibers innervate hairy and globular skin and the combination of these fibers allows us to experience the different modalities of the sense of touch (Abraira and Ginty, 2013; Roudaut et al., 2012). In hairy skin, these fibers innervate hair follicles, where they form longitudinal and circumferential lanceolate endings (Abraira and Ginty, 2013). Some C-fibers also form free nerve endings which contain both noxious mechanoreceptors and low threshold mechanoreceptors (Roudaut et al., 2012). In conjunction with its identification in large diameter DRG neurons, Piezo2 was also identified in nerve endings in the hairy skin of mice. Piezo2 was identified in longitudinal and circumferential lanceolate endings innervating hair follicles (Ranade et al., 2014). Glabrous skin contains slow and rapidly adapting receptors. Merkel cells (modified epidermal cells) form a complex with the slowly adapting LTMRs while Meissner and Pacinian corpuscles form part of rapidly adapting complexes (Abraira and Ginty, 2013). Sensory fibers form specialized complexes with modified epidermal cells for example the Merkel cell-neurite complex. In a series of studies published recently it was established that Merkel cells are inherently mechanosensitive and express Piezo2. The sensory afferent (also expressing Piezo2) forms a complex with Merkel cells which could be the basis for highly sophisticated responses to mechanical stimuli (Ikeda et al., 2014; Maksimovic et al., 2014; Woo et al., 2014). In order to understand more about the functions of Piezo2, Ranade and colleagues, generated an inducible Piezo2 knockout mouse (constitutive Piezo2 deletion is lethal in mice Dubin et al. (2012) and Ranade et al. (2014)). The inducible deletion of Piezo2 was done in sensory neurons and epidermal Merkel cells using the Advillin promotor. These animals show a pronounced loss of touch sensation, without any 
significant differences in the nociceptive functions (Ranade et al., 2014). Further, Piezo2 was also shown to be the principle mechanotransduction channel for proprioception (both body and head) again using mouse lines that specifically deleted Piezo2 in proprioceptive neurons. These mice showed uncoordinated body movements and abnormal limb positions, both signs of proprioceptive defects (Florez-Paz et al., 2016; Woo et al., 2015). Interestingly, Piezo2 was found to be required for mechanotransduction in human stem cell-derived touch receptors (Schrenk-Siemens et al., 2014) and in a subsequent study two patients with loss-of-function mutations in Piezo2 were identified. These patients showed pronounced deficits in touch, vibration and proprioception, while mechanical and thermal pain threshold were comparable to controls (Chesler et al., 2016). This provides additional evidence that mammalian Piezo2 is essential for tactile sensory information, but may not play an essential role in nociception.

Despite the above mentioned studies, new evidence is accumulating for the role of Piezo2 in nociception. Firstly Piezo2 expression was found in small diameter DRG neurons where it overlaps with TRPV1, a known mediator of nociception (Coste et al., 2010). Additionally, proalgesic peptide, bradykinin could potentiate Piezo2 mediated MA currents. In case of nociceptors, such heightened function contributes to increased sensitivity to pain (hyperalgesia). The role of TRPV1 in thermal hyperalgesia is well defined, but the potentiation of Piezo2 by inflammatory mediators, suggests that its sensitization may play a role in mechanical hyperalgesia (Dubin et al., 2012). Another line of evidence came from Eijkelamp and colleagues, who showed that Epac1 (cyclic AMP sensor) causes potentiation of RA-MA (Piezo2 mediated currents) and contributes to mechanical allodynia (when innocuous stimuli are perceived as painful). Epac1 is a guanine nucleotide exchange factor, which is required for the development of allodynia in the mouse model of neuropathic pain (Eijkelkamp et al., 2013). Further, Piezo2 was shown to play a role in stimulus-dependent mechanical hyperalgesia in endothelial cells (Ferrari et al., 2015). This shows that though Piezo2 may not be the main channel involved in nociception, its regulation may play a role in nocifensive behaviors like mechanical allodynia and hyperalgesia (Lolignier et al., 2014).

\subsubsection{Pathology associated with Piezo2}

As mentioned in the last section, complete ablation of Piezo2 in mice is perinataly lethal (Dubin et al., 2012; Ranade et al., 2014), presumably due to defects in lung development. But several mutations have been described in human Piezo2, some of which produce inactive channels. Interestingly, humans only show sensory-motor phenotypes upon lack of functional Piezo2 (in clear contrast to mice) (Chesler et al., 2016). Distal arthrogryposis type 5 (DA5), Gordon Syndrome and Marden-Walker syndrome are some of the 
conditions directly linked to Piezo2. The patients present with joint and muscle contractures, cleft palate and developmental delay among other symptoms (Coste et al., 2013; McMillin et al., 2014). The differences between the mouse and human Piezo2 and its deletion still remain to be understood.

\subsection{Protein complexes associated with ion channels}

\subsubsection{Ion channel function and protein complexes}

Ion channels are integral membrane proteins usually containing a pore domain spanning the lipid bilayer and allowing the diffusion of ions. These channels are gated by multiple mechanisms such as ligands, voltage or mechanical stimuli. An important requirement for these channels is to modulate channel opening and closing in response to extra or intracellular signals. It is widely known that ion channels often exist as part of dynamic multi-protein complexes. These interacting proteins could either be subunits required for the channel assembly and function, or they could be additional proteins that associate with the ion channels and regulate its properties. Other parts of this complex could be proteins relevant for the targeting of ion channels, membrane modifying proteins, cytoskeletal elements, all of which are essential for channel function and regulation (Levitan, 2006).

\subsubsection{Protein complexes in mechanotransduction}

As with other ion channels, mechanosensitive ion channels also appear to exist as part of protein complexes. Though the knowledge of these complexes in mammals is incomplete, evidence from $C$. elegans and Drosophila is very strong. In $C$. elegans the mechanotransducing channel complex MEC-10, MEC-6 and MEC-4 are supported by extracellular (MEC-5) and intracellular (MEC-7, MEC-12) anchor proteins. Extracellular linker MEC-9 links the channel to the extracellular anchor and stomatin-like protein MEC2 links the channel to cytoplasmic microtubules (Figure 3A). In Drosophila, a very similar arrangement is seen where the transduction channel (NompC) is linked to an extracellular anchor and cytoplasmic microtubules through linker proteins (Figure 3B). And in the mammalian auditory system, despite the debate around the transduction channel (Ranade et al., 2015), several regulators of the complex have been identified (Figure 3C; Gillespie and Walker (2001)). The question if similar complexes exist in mammalian somatosensory mechanotransduction remains open. 
A

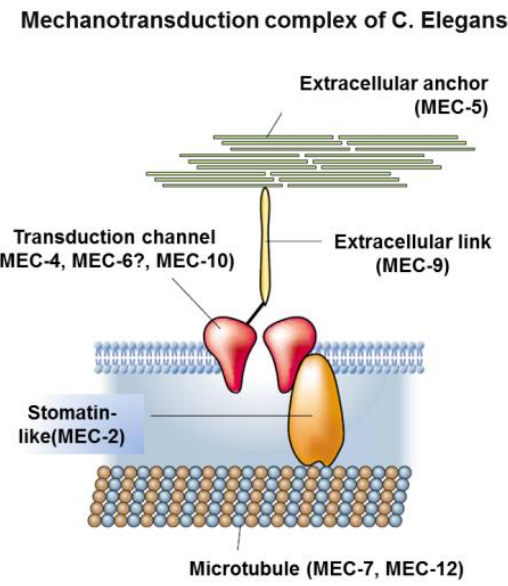

B

Mechanoreceptor complex of Drosophila

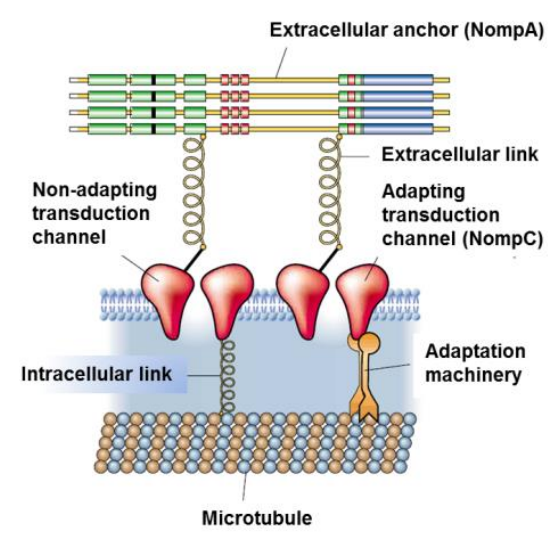

C

Mechanoreceptor complex of mammalian audition

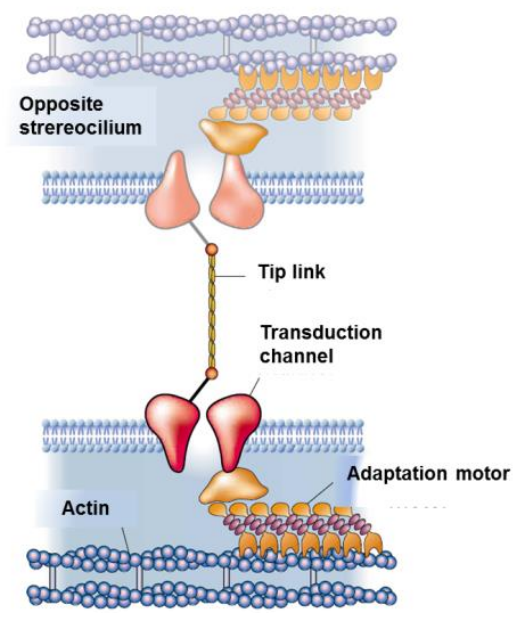

Figure 3: Illustrations of mechanosensitive channel complexes

(A) Proposed model of mechanotransduction complex in C. elegans indicating the hypothetical locations of MEC proteins that form integral components of the mechanosensing apparatus in the mechanoreceptors.

(B) Proposed molecular model of mechanotransducing machinery in the ciliated Drosophila mechanoreceptor and (C) proposed molecular model of the mammalian auditory inner hair cell mechanotransduction apparatus. Adapted from Gillespie and Walker (2001)

\subsubsection{Protein complexes in mammalian somatosensation}

Though the protein complexes associated with mammalian somatosensation have not been completely discerned, there is evidence for some proteins that modulate mechanotransduction in this system. The first such evidence was the identification of Stoml3 (Stomatin-like protein 3, SLP3) as a crucial protein for somatic touch. Stoml3 is a homolog of the $C$. elegans MEC-2 protein which is required for touch receptor function. Stoml3 is required for the expression of mechanosensitive currents in a subset of DRG neurons as well as for mechanical sensitivity in vivo (Hu et al., 2010; Wetzel et al., 2007). 
In subsequent studies, after the discovery of the Piezo2 protein it was shown that Stoml3 modulates Piezo2 activity by influencing local membrane properties (Poole et al., 2014; Qi et al., 2015). Another interesting modulator of somatic touch is Runx1, a transcription factor which was shown to be essential for the expression of VGLUT2 and TH (tyrosine hydroxylase) in LTMRs. It was also required for the formation of longitudinal lanceolate endings, which are LTMRs innervating the hair follicles in the skin. Interestingly, the role of Runx1 in establishing mechanosensitivity in LTMRs is through regulation of Piezo2 expression (Lou et al., 2013). Additionally, potassium channel subunits (Kcna1/Kv1.1 and Kcna2/Kv1.2) were shown to regulate adaptation of distinct mechanoreceptor populations (Hao et al., 2013). The most recently identified regulator of somatic touch is CaMKII $\left(\mathrm{Ca}^{2+} /\right.$ calmodulin-dependent kinase II). It was shown to mediate activity dependent plasticity in somatic touch and adequate levels of CaMKII function was essential to prevent allodynia like conditions (Yu et al., 2015).

\subsubsection{Piezo2 associated proteins (Piezo2 interactors)}

As mentioned above very few modulators of somatic touch and interactors of Piezo2 have been identified so far (Figure 4). The first study that systematically identified the interactors of native Piezo2 was reported by our group in 2016. The study used immunoprecipitation followed by highly sensitive liquid chromatography (LC)-coupled tandem mass-spectrometry (MS/MS) to identify interactors of native Piezo2 from DRG tissue of mice (summarized in Figure 5; Narayanan et al. (2016)). For this screen firstly, commercial Piezo2 antibodies were tested for specificity in immunoprecipitating native Piezo2 from sensory neurons. Figure 6A depicts the specificity of Piezo2 Ab1 (Piezo2) in detecting Piezo2 compared to Piezo2 Ab-2 and IgG controls. Then, using the positive Piezo2 antibody, native Piezo2 was immunoprecipitated from DRGs and the resulting mixture was subjected to mass-spectrometry. Through this screen 36 proteins were identified as potential interactors of Piezo2 in somatosensory neurons (Figure 6B). Candidates identified in the screen were analyzed using Gene ontology analysis using the DAVID bioinformatics resource and STRING visualization tool (Figure 6C and 6D; Huang da et al. (2009) and Huang et al. (2009)). The identified proteins belonged to various categories, including cellular trafficking, cytoskeletal proteins and ion channels. Identification of known modulators of somatosensory mechanotransduction like Kcna1 and 2 and CamKII (as discussed in the previous section) suggests that this screen successfully identified proteins relevant to somatosensory mechanotransduction. In the current study, two candidates identified from this screen were characterized as functional interactors of mPiezo2. 


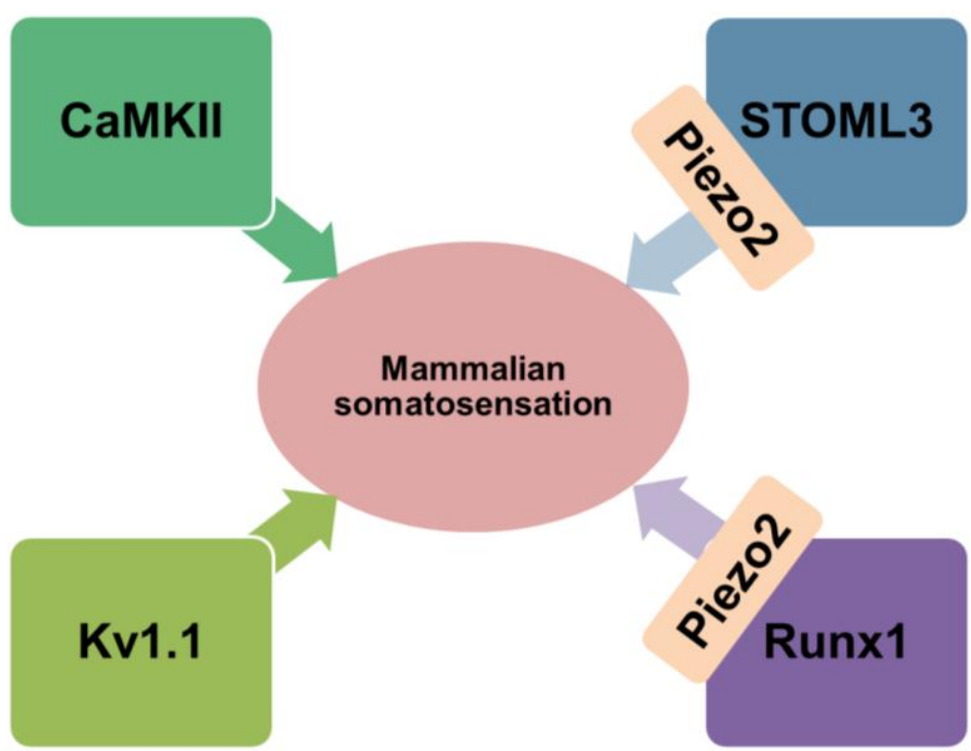

Figure 4: Identified components of mammalian somatosensory complex.

CaMKII and Kv1.1 modulate adaptation and plasticity in the somatosensory system. STOML3 is shown to directly interact with Piezo2 and is essential for somatic touch. Runx1 is a transcription factor required for proper formation of specialized peripheral endings, through regulation of Piezo2 expression.

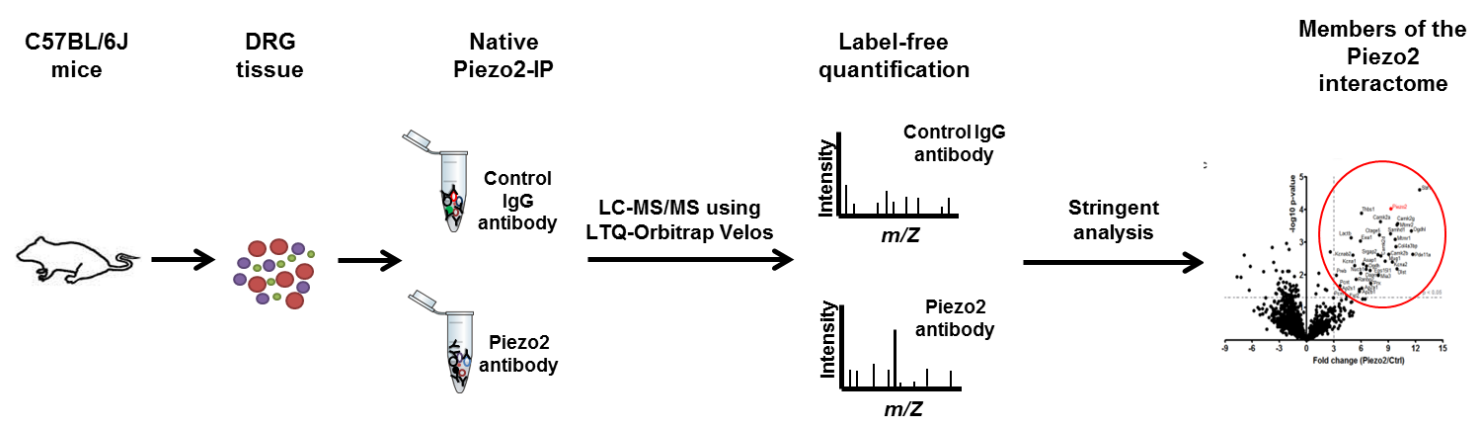

Figure 5: Scheme of mass-spectrometry (MS) based interactomics approach to identify Piezo2 interactors

Interactors of native Piezo2 were identified from sensory neurons of mouse using immunoprecipitation followed by mass-spectrometry. DRG tissue was isolated from WT C57BL/6J mice and subjected to immunoprecipitation using a positively tested anti-Piezo2 ab. The immunoprecipitate was subjected to LCMS/MS (Liquid chromatography coupled mass-spectrometry). Label free quantification with stringent statistical criteria based on significance scores was used to discriminate hits from the background. Reprinted (adapted) with permission from Narayanan et al. (2016). Copyright (2016) American Chemical Society. 
A

\begin{tabular}{|c|c|c|c|c|c|c|c|c|c|}
\hline & 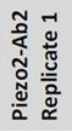 & 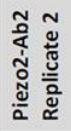 & 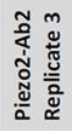 & 总 & 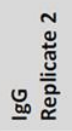 & 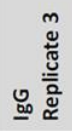 & 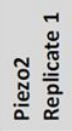 & 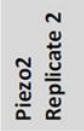 & 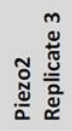 \\
\hline $\begin{array}{l}\text { Piezo2 } \\
\text { (a8cD54) } \\
\text { MS/Ms } \\
\text { Count }\end{array}$ & 0 & 0 & 0 & 0 & 0 & 0 & 33 & 84 & 63 \\
\hline
\end{tabular}

B

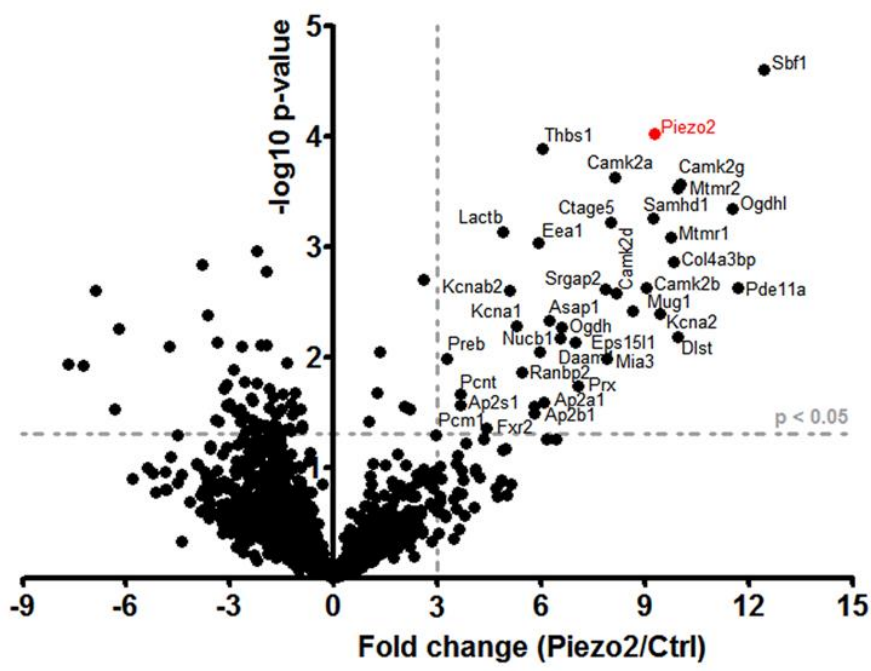

C

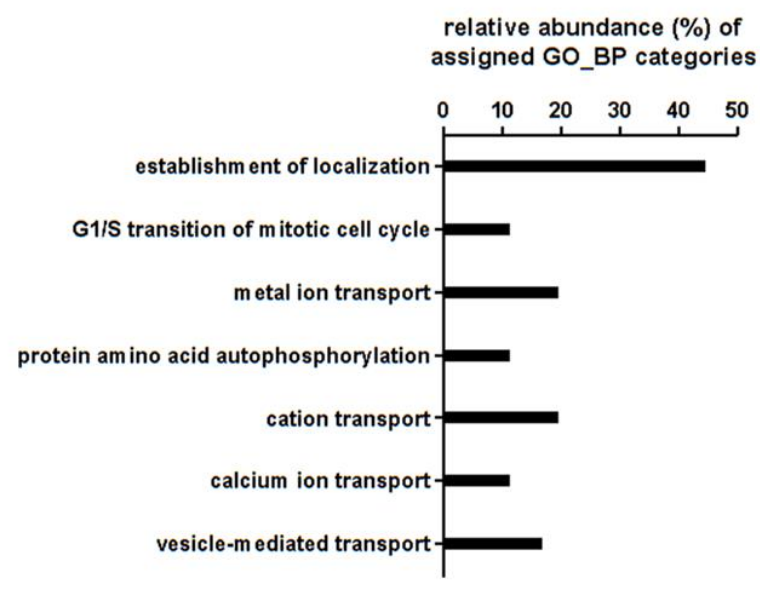

D

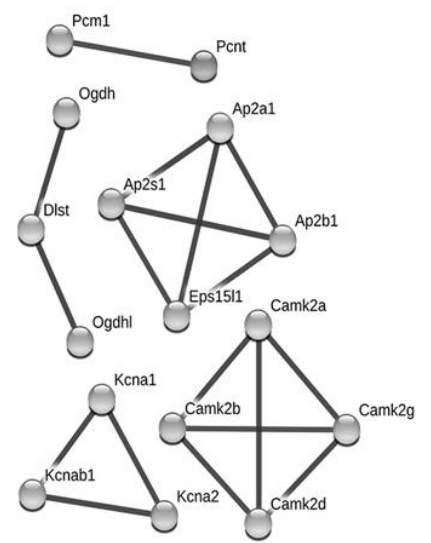

Figure 6: Quantitative mass-spectrometry (MS) based interactomics approach to identify Piezo2 interactors (A) Quantitative mass-spectrometry using specific Piezo2 antibody (Piezo2) successfully detects Piezo2 peptides in sample but not in controls (IgG and negative Piezo2 antibody (Piezo2-Ab2)) (B) Volcano plot of mean fold change versus negative log10 p-value, showing the members of the Piezo2 interactome. The top right quadrant illustrates candidates which passed the statistical criteria to be described as true interactors. (C) Assigned Gene ontology (GO) biological process categories and the relative abundance of each category in the above identified Piezo2 interactome. (D) STRING visualization of physical and functional associations which were identified between candidates of the Piezo2 interactome. Reprinted (adapted) with permission from Narayanan et al. (2016). Copyright (2016) American Chemical Society. 


\subsection{Potential Piezo2 interactors: Pericentrin (Pcnt) and Mtmr2}

\subsubsection{Pericentrin}

Pcnt was first identified in mice as a $220 \mathrm{kDa}$ protein, found in centrosomes and noncentriolar microtubule organizing centers (MTOC) (Doxsey et al., 1994). It was later identified that Pcnt exists as three splice variants, namely Pcnt A (220-250 kDa), Pcnt B (>350 kDa) and Pcnt S (220 kDa) (Figure 7; Miyoshi et al. (2006) and Mühlhans and GießI (2012)). Pcnt is a large coiled-coiled protein, which is expressed in the pericentriolar material (PCM) surrounding the centrosome and in basal bodies of primary cilia (Delaval and Doxsey, 2010). It serves as a multifunctional scaffold and plays a role in cell cycle regulation, mitotic spindle assembly and orientation, and primary cilia assembly and development (Delaval and Doxsey, 2010). Due to the key cellular processes Pcnt is involved in, it is also linked to various disorders. Some of these disorders include, microencephalic osteodysplastic primodial dwarfism (MOPDII), chronic myeloid leukemia (CML) and mental disorders like schizophrenia (Delaval and Doxsey, 2010). Various binding partners of Pcnt have been identified to date. The motor protein dynein is involved in the transport of Pcnt to the centrosome and the assembly of the mitotic spindle. The process is mediated by Pcnt binding of dynein light intermediate chain (Purohit et al., 1999). Another interesting interactor of Pcnt is the pericentriolar material 1 protein (PCM-1), which is required for the localization of Pcnt to the centrosomes (Dammermann, 2002; Li et al., 2001). It is relevant to note here that PCM-1 was also identified in the native Piezo2 interactome described earlier (Narayanan et al., 2016). Additionally, Pcnt has also been implicated in the regulation of secretory vesicle docking and insulin secretion in pancreatic $\beta$-cells through its interaction with F-actin (Jurczyk et al., 2010; Zu et al., 2015). Further, in its function as a potential scaffold at the base of primary and motile cilia, Pcnt interacts with cation channel polycystin-2 (PC2) (Jurczyk et al., 2004). Interestingly, PC-1 and 2 have been suggested to function in mechanotransduction in the primary cilium of renal epithelia (Nauli et al., 2003).

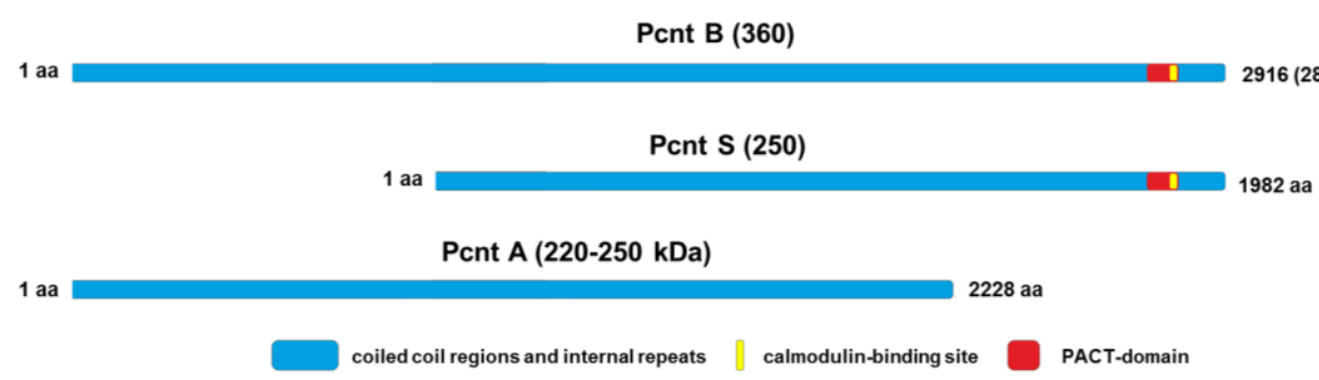

\section{Figure 7: Pericentrin structure and isoforms}

Illustration of Pericentrin isoforms, Pericentrin A, B and S. In mice, Pcnt B is the largest isoform with a molecular weight of $360 \mathrm{kDa}$. Two smaller isoforms Pcnt S (250 kDa) and Pcnt A (220-250kDa) have also been described in mice. Adapted from Mühlhans and GießI (2012). 


\subsubsection{Pericentrin in sensory systems}

Pcnt has also been shown to play a role in sensory systems. In mouse photoreceptors, Pcnt is expressed in the connecting cilium, which bridges the outer and inner segments of the photoreceptor. Pcnt localizes to the basal body complex where it is involved in transport of proteins between the two segments (Mühlhans et al., 2011). Pcnt is also expressed in the olfactory cilia of chemosensory neurons in the nasal olfactory epithelium. Mutations in Pcnt cause malformation of the cilia and reduce olfactory performance (Miyoshi et al., 2009). Furthermore, Pcnt mutant mouse displays an impaired interneuron migration to the olfactory bulb (Endoh-Yamagami et al., 2010). Another interesting role for Pcnt was described in Drosophila. The Drosophila Pcnt-like protein (D-PLP), is essential for cilia and flagella function in flies, while it appears to be dispensable for mitosis. D-PLP mutant flies show defects in mechanosensory cilia and are phenotypically, uncoordinated (Martinez-Campos et al., 2004).

Through this study I ask if Pcnt interacts with mPiezo2 and plays a role in mammalian somatosensory mechanotransduction.

\subsubsection{Myotubularin related protein-2 (Mtmr2)}

Myotubularin-related proteins (MTMRs) are a family of conserved protein tyrosine/dual specificity phosphatase-like phosphatases (Laporte et al., 1998). There are 14 members in the family, of which eight are catalytically active while six are catalytically inactive members (Lorenzo et al., 2006). MTMR proteins share certain structural features, namely an N-terminal GRAM domain, a PTP-like catalytic domain, a coiled-coil domain and Cterminal domains such as PDZ or FYVE (Begley et al., 2006; Lorenzo et al., 2006). Mtmr2 is a 643 amino acid long, catalytically active member of this family (Figure 8A; Bolino et al. (2002)). Mtmr2 has been studied largely with regard to its role in the peripheral neuropathy Charcot-Marie-Tooth disease Type 4B1 (CMT4B1) (Bolino et al., 2004; Bolis et al., 2005; Previtali et al., 2007). Though MTMRs are structurally similar to the protein tyrosine phosphatases, they do not use phosphoproteins as substrate. The MTMR family, specifically dephosphorylates the D3 position of phosphoinositide phosphates like phosphatidylinositol 3-phosphate (PI(3)P) and phosphatidylinositol $(3,5)$ bisphosphate $\left(\mathrm{PI}(3,5) \mathrm{P}_{2}\right)$ (Begley et al., 2006). Mtmr2 is also capable of dephosphorylating both $\mathrm{PI}(3) \mathrm{P}$ and $\mathrm{PI}(3,5) \mathrm{P}_{2}$, but shows higher efficiency towards $\mathrm{PI}(3,5) \mathrm{P}_{2}$ (Figure 8B; Berger et al. (2002)). As expected from its role in peripheral neuropathy, Mtmr2 shows strong expression in the peripheral nervous system (studies in mice and rats). Dorsal root ganglia and spinal roots show abundant Mtmr2 mRNA expression starting from early developmental stages (Bolino et al., 2002). Mtmr2 has 
been detected in the cytoplasm of both myelin forming and non-myelin forming Schwann cells in the sciatic nerve of mice and rats. It also shows strong expression in both sensory and motor neurons that send axons to the peripheral nerves. The cell bodies and axons of these neurons showed Mtmr2 staining (Previtali et al., 2003). In terms of sub-cellular localization, Mtmr2 is known to express in the cytosol or associate with the peripheral membranes. In some cases it has been shown to have a nuclear localization though the role of this remains unclear (Previtali et al., 2007; Previtali et al., 2003). Of note, Mtmr2 when overexpressed in mammalian cells, localizes to the cytosolic compartment $(\mathrm{Ng}$ et al., 2013).

A

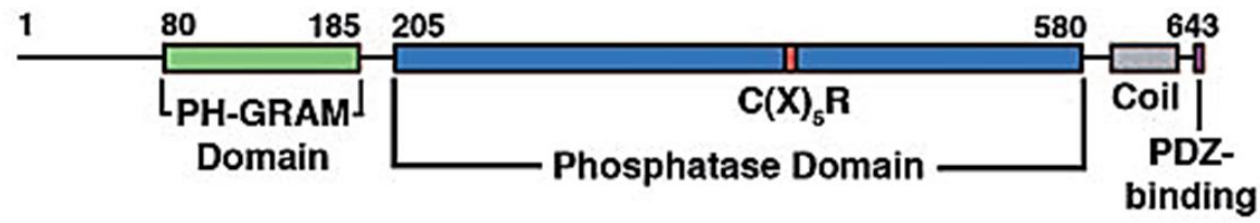

B

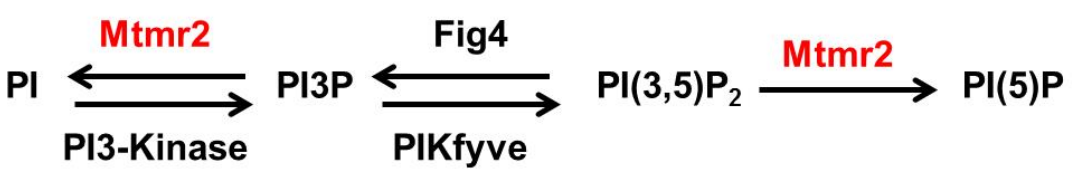

Figure 8: Mtmr2 structure and phospholipid catalysis pathway

(A) Mtmr2 is the catalytically active member of the MTMR phosphatase family. Illustration of Mtmr2 structure depicting the different structural motifs; PH-GRAM domain, phosphatase domain and PDZ binding domain. Adapted from Begley et al. (2006). (B) Illustration of the phosphatidylinositide pathway, which involves Mtmr2 as a 3-phosphatase.

\subsubsection{Mtmr2 interactors}

Because of the important role Mtmr2 plays in CMT4B1, it has been the subject of various studies to understand its cellular function and how it could contribute to disease progression. The presence of active and inactive phosphatases in the MTMR family has led to speculation that they may interact to regulate each other. Two such interactions have been characterized for Mtmr2, namely with Mtmr5 (Sbf1) and Mtmr13 (Sbf2) (Clague and Lorenzo, 2005; Lorenzo et al., 2006). Mtmr5 interacts with Mtmr2 through the coiled-coil domain and increases its enzymatic activity. Mtmr5 (catalytically inactive phosphatase) is also responsible for dictating the subcellular localization of Mtmr2 (Kim et al., 2003). Interestingly, Mtmr5 (Sbf1) was also identified as a potential member of the Piezo2 interactome in sensory neurons (Narayanan et al., 2016). The other interaction 
between MTMR family members is between Mtmr2 and Mtmr13 (Sbf2). In this case, Mtmr2 is responsible for localizing and stabilizing Mtmr13 (catalytically inactive phosphatase) at endomembranes and both proteins are dependent on each other to maintain their levels in the cell ( $\mathrm{Ng}$ et al., 2013). Besides its interactions with members of the MTMR family of proteins, Mtmr2 also interacts with other proteins both in neurons and Schwann cells. Using a yeast two-hybrid screen, neurofilament light chain protein (NF-L) was shown to interact with Mtmr2 in neurons and Schwann cells. It was postulated that NF-L may be involved in targeting Mtmr2 to its site of action, and aide the role of Mtmr2 in plasma membrane remodeling (Previtali et al., 2003). Another interaction characterized in Schwann cells, was with Discs large 1(Dgl1). This interaction is responsible for negative regulation of membrane addition and its disruption may be responsible for the characteristic myelin out folding phenotype of CMT4B1 (Bolis et al., 2009). Additionally, Mtmr2 was also shown to localize to synapses of excitatory neurons through interactions with PSD-95. Loss of Mtmr2 from these neurons causes lowered synaptic density and function. Interestingly, this interaction requires a catalytically active phosphatase, indicating a role for $\mathrm{PI}(3) \mathrm{P}$ or $\mathrm{PI}(3,5) \mathrm{P}_{2}$ in the interaction (Lee et al., 2010). In a subsequent study, Mtmr2 was shown to interact with Fig4. Fig4 plays a role in synthesis of $\mathrm{PI}(3,5) \mathrm{P}_{2}$ while Mtmr2 dephosphorylates it. The study showed that Fig4 downregulation was able to counteract the effects of Mtmr2 deletion in mice (Vaccari et al., 2011). In summary, Mtmr2 plays an important role in sensory neurons and Schwann cells and its cellular targets $\mathrm{PI}(3) \mathrm{P}$ and $\mathrm{PI}(3,5) \mathrm{P}_{2}$ are relevant for these roles.

\subsubsection{Phosphatidylinositol lipids}

Phosphorylated phosphatidylinositol (PIP) lipids regulate a variety of cellular processes. Seven members of this family have been identified in mammals of which the cellular role of $\mathrm{PI}(4,5) \mathrm{P}_{2}$ is most studied (Jin et al., 2016). The precursor to these PIPs is the abundant membrane lipid phosphatidylinositol $(\mathrm{PI})$. Tightly regulated kinases and phosphatases control the synthesis and turnover of these PIPs (McCartney et al., 2014a). As mentioned above, Mtmr2 is involved in the dephosphorylation of two members of this family i.e. $\mathrm{PI}(3) \mathrm{P}$ and $\mathrm{PI}(3,5) \mathrm{P}_{2}$. $\mathrm{PI}(3,5) \mathrm{P}_{2}$, is a member of the above family but has a relatively low abundance in the cell compared to other PIPs. In mammalian cells, it accounts for approximately $0.05 \%-0.1 \%$ of the total phosphatidylinositol lipids which is about 125-fold less abundant than PI(4,5) $\mathrm{P}_{2}$ (Jin et al., 2016; McCartney et al., 2014a). $\mathrm{PI}(3,5) \mathrm{P}_{2}$ levels show dynamic and transient changes upon exposure to cellular stress such as osmotic shock in both yeast and mammalian cells. This suggests a role for $\mathrm{PI}(3,5) \mathrm{P}_{2}$ in cellular homeostasis and adaptation (Jin et al., 2016; McCartney et al., 2014a). The dynamic changes in the levels of this PIP also indicate a tightly regulated 
synthesis and turnover. In mammalian cells, only one pathway is known to synthesize $\mathrm{PI}(3,5) \mathrm{P}_{2}$, which involves the Fab1/PIKfyve kinase. This kinase is further regulated by scaffolding proteins Vac14 and phosphatase Fig4 (Zolov et al., 2012). MTMRs, with their 3-phosphatase activity are involved in the dephosphorylation of $\mathrm{PI}(3,5) \mathrm{P}_{2}$ to generate $\mathrm{PI}(5) \mathrm{P}$. Mouse Mtmr2 has been shown to function with the Fab1/PIKfyve complex to generate PI(5)P (Vaccari et al., 2011).

\subsubsection{3 $\mathrm{PI}(3,5) \mathrm{P}_{2}$ regulatory functions}

Despite its low abundance, $\mathrm{PI}(3,5) \mathrm{P}_{2}$ plays an important regulatory role in cells. A study by Dong and colleagues, identified TRPML1 as a channel regulated by $\mathrm{PI}(3,5) \mathrm{P}_{2}$ (Dong et al., 2010). Of note, TRP channels have been known to be regulated by the more abundant $\mathrm{PI}(4,5) \mathrm{P}_{2}$ (Borbiro et al., 2015). The study showed that $\mathrm{PI}(3,5) \mathrm{P}_{2}$ is able to activate TRPML1 in the endo-lysosomal membrane and further went on to show that $\mathrm{PI}(3,5) \mathrm{P}_{2}$ binds directly to the N-terminal of TRPML1 (Dong et al., 2010). Another study showed that $\mathrm{PI}(3,5) \mathrm{P}_{2}$ levels are dynamically regulated in active neurons. It implicated $\mathrm{PI}(3,5) \mathrm{P}_{2}$ in the endocytosis and recycling of AMPAR (AMPA type glutamate receptor), which is in turn linked to activity dependent changes in synaptic strength (McCartney et al., 2014b). Besides these, various studies have looked at potential $\mathrm{PI}(3,5) \mathrm{P}_{2}$ binding motifs and identified WD40 domain containing proteins for example Atg18, Atg21 etc. as potential $\mathrm{PI}(3,5) \mathrm{P}_{2}$ binding proteins. $\mathrm{PI}(3,5) \mathrm{P}_{2}$ also binds to and activates ryanodine receptors in the skeletal and cardiac muscles, to stimulate calcium release (McCartney et al., 2014a).

Taken together, it can be seen that though low in abundance, $\mathrm{PI}(3,5) \mathrm{P}_{2}$ can play crucial roles in regulation of cellular processes and it remains to be seen if it has a role in somatic touch.

\subsection{Aims of the study}

Tactile perception has remained a mystery in sensory neuroscience for a long time. While all other sensory systems are relatively well characterized from their molecules to processing pathways, tactile sensation and perception remained obscure.

Only in 2010, the Piezo family was identified as bona fide mammalian mechanosensitive ion channels. Of these, Piezo2 has subsequently been implicated in light touch and proprioception making it the much needed stepping stone for understanding more about this fundamental sense. The complexity of tactile sensation requires the components of the system to be tuned to a wide range of stimuli. Anatomical features including high and low threshold receptors and slow and rapid adaptors are involved in the coding of 
different aspects of touch. Besides these, the fundamental ion channel i.e. Piezo2 also needs to be regulated and modulated for further signaling. That, mechanically gated ion channels are tightly regulated by ion channel complexes is already known from C.elegans and Drosophila, but the evidence of such a complex is missing in mammals. Only few direct interactors of Piezo2 have been reported so far, namely STOML3 and Runx1. In 2016, through an interactomics based approach, 36 potential interactors of Piezo2 were identified.

In the course of this thesis, I aimed to characterize two candidates identified in this screen, namely Pericentrin (Pcnt) and Myotubularin related protein-2 (Mtmr2) as potential Piezo2 interactors and modulators.

The first candidate Pcnt, is best known for its role in cell cycle regulation and assembly of primary cilia. Through its role in the microtubule organizing complex, Pcnt has been involved in olfaction, vision and mechanosensation. In mammals however, this protein has not been characterized in the somatosensory systems. For this part of the study, my aim was to characterize Pcnt in the mouse peripheral somatosensory system and study its effect on Piezo2.

The second part of this study involved the characterization of Mtmr2-Piezo2 interaction. Mtmr2 is a phosphatase known largely due to its role in the sensory motor neuropathy Charcot-Marie-Tooth disease type 4B1 (CMT4B1). Functionally, Mtmr2 is involved in the turnover of phosphatidylinositide phosphates (PIPs) particularly, $\mathrm{PI}(3) \mathrm{P}$ and $\mathrm{PI}(3,5) \mathrm{P}_{2}$. The aim of this part of the study was to characterize the interaction of Piezo2 and Mtmr2 and identify if $\mathrm{PI}(3) \mathrm{P}$ and $\mathrm{PI}(3,5) \mathrm{P}_{2}$ lipids contribute to Piezo2 channel function or modulation.

Overall, the questions asked in this study aim to uncover novel aspects of Piezo2 regulation and modulation. 


\section{METHODS}

\subsection{Verification of Piezo2-candidate interaction}

\subsubsection{Transient transfection of HEK293 cells}

HEK293 cells were cultured in T-75 flasks at $37^{\circ} \mathrm{C}$ and $5 \% \mathrm{CO}_{2}$, up to $80-90 \%$ confluency. Growth media consisting of DMEM with Glutamax supplemented with $10 \%$ Fetal Bovine Serum and 1\% Penicillin/Streptomycin was used for the culture.

For transfection, cells were plated onto $15 \mathrm{~cm}$ cell culture dishes or $10 \mathrm{~mm}$ poly-D-lysine coated coverslips. Cells were transfected with desired plasmids using FugeneHD transfection reagent. Transfection reagent master mix included Opti-MEM, DNA and FugeneHD (DNA:Fugene ratio 1:3). Transfection mix was pipetted evenly over the cells and they were allowed to grow for 48 hours at $37^{\circ} \mathrm{C}$ and $5 \% \mathrm{CO}_{2}$. Transfection efficiency was checked by GFP fluorescence (from Piezo2-GST IRES GFP expression), observed using the Zeiss Axio Observer Z1 inverted microscope. Details of the plasmids used in the study are provided in supplementary table 1.

\subsubsection{Co-immunoprecipitation of Piezo2 and candidate from HEK293 cells}

HEK293 cells were transfected with Piezo2, co-transfected with mock or candidate $(4 \mu \mathrm{g}$ each per dish), using FugeneHD transfection reagent. The cells were grown for 48 hours prior to harvesting. Cells were harvested in 1X PBS and lysed in buffer containing $5 \mathrm{M}$ $\mathrm{NaCl}, 1 \mathrm{M}$ Tris buffer, $1 \mathrm{M}$ Sucrose, 1M DTT, 1\% DDM and protease inhibitor. The lysate was incubated overnight with glutathione coupled beads (Pierce Glutathione Magnetic beads) or Dynabeads Protein G coupled to the GST antibody. Elution was performed using buffer containing glutathione, reducing agent (NuPAGE sample reducing agent) and LDS (NuPAGE LDS sample buffer).

Input and eluted fractions were loaded on a 3-8\% Tris-acetate gel for gel electrophoresis. Protein was transferred onto nitrocellulose membrane using the iBlot Dry Blotting system (Life Technologies). The membrane was blocked with 5\% non-fat milk for 30 minutes before probing with primary antibodies (diluted in 1\% non-fat milk). Membrane was washed with 1X PBS and probed with secondary antibody coupled to Alexa680 (diluted in $1 \%$ non-fat milk). Membrane was washed again and imaged using an Odyssey Infrared System (LI-COR). Details of antibodies used in this study are provided in supplementary table 2. 


\subsubsection{Immunohistochemistry}

\section{Experimental methods}

C57BL/6J mice or Piezo2 ${ }^{\text {GFP }}$ (Woo et al., 2014) (between 8-9 weeks) were either euthanized with $\mathrm{CO}_{2}$ or perfused with $4 \%$ PFA. DRG and backskin were isolated from the animals and post fixed with 4\% PFA and cryoprotected with $30 \%$ sucrose overnight. The tissue was then embedded in optimal cutting temperature medium and sectioned with a cryostat into $10 \mu \mathrm{m}$ thick sections, mounted onto SuperFrost Plus slides and stored at $80^{\circ} \mathrm{C}$. For the staining, the slides were thawed at room temperature for 30 minutes. Tissue region on the slide was outlined using a hydrophobic pen and allowed to dry. The slides were then washed with 1X PBS and incubated with blocking solution (3\% serum, $0.4 \%$ TritonX-100 in $1 \mathrm{X}$ PBS) for 30 minutes at room temperature. Following this, the slides were incubated with primary antibody solution (primary antibody dissolved in 1\% serum, $0.1 \%$ Triton- 100 in $1 \mathrm{X}$ PBS), overnight at $4^{\circ} \mathrm{C}$ in a humidified chamber. After the overnight incubation, slides were washed thrice with 1X PBS. The slides were then incubated with the secondary antibody solution for 2 hours at room temperature in a humidified chamber. The slides were then washed again with 1X PBS and mounted with Slowfade Gold antifade reagent with DAPI and allowed to dry before imaging. In case of antibodies raised in mouse, the sections were incubated with donkey anti-mouse Fab fragments for 60 minutes at room temperature prior to staining with primary antibody. This prevented non-specific binding of anti-mouse secondary antibody to the tissue. For secondary controls, slides were stained with secondary antibody only. A list of all the antibodies used in this study is provided in Table 2.

\section{Image acquisition}

The slides were imaged using either the Zeiss Axio Observer Z1 inverted microscope or a Zeiss 510 meta confocal laser scanning microscope (CLSM). 10X, 20X air objectives or $40 \mathrm{X}$ oil objectives were used. The imaging was always done keeping conditions constant for samples from the same experiments. Secondary controls were always imaged using the same conditions as sample sections.

\section{Analysis}

The analysis of immunostainings was done using NIH ImageJ (Schindelin et al., 2015). To identify the immuno-label positive cells, threshold was defined as: mean intensity (AU) $+3^{*}$ standard deviation of negative cells (10 random unstained neurons were chosen as negative cells) for each channel. Positive cells were counted using the "cell counter" tool (NIH ImageJ). For analysis of immunolabel in nerve endings in the backskin sections, 
optical sections were acquired with a Zeiss 510 meta confocal laser scanning microscope (CLSM) using the 40X oil objective. Noise removal and out of focus signal reassignment was conducted with the Huygens Essential deconvolution package (version 15.10, Scientific Volume Imaging) by applying a theoretical point spread function and the classical maximum likelihood estimation restoration method. Images were further processed with NIH ImageJ or Imaris 8.2.0 (Bitplane). Values are reported as mean \pm SEM or percentages. Statistical analysis was done using unpaired t-test using GraphPad Prism 6.01(San Diego).

\subsubsection{Proximity ligation assay (PLA)}

\section{Experimental methods}

PLA was carried out as described in Hanack et al. (2015) with minor modifications. DRG neurons or HEK293 cells were plated on MatTek dishes coated with poly-D-lysine $(1 \mathrm{mg} / \mathrm{mL})$ and laminin $(20 \mu \mathrm{g} / \mathrm{mL})$. HEK293 cells were transfected with Piezo2 and cotransfected with mock or candidate construct $(0.5 \mu \mathrm{g}$ each per dish). Cells were allowed to express the constructs for 48 hours before staining. Prior to staining, cells were washed with 1 X PBS and fixed with 4\% PFA for 10 minutes at room temperature. Thereafter cells were blocked in Duolink blocking solution for 2 hours at room temperature. Cells were then incubated with primary antibody (diluted in Duolink antibody diluent), overnight at $4^{\circ} \mathrm{C}$. [Preparation of antibody-conjugated PLA probe using the Duolink In SituProbemaker kit: goat anti-chicken Alexa Fluor 647 (1 mg/mL in 1X PBS) was mixed with Conjugation Buffer and added to lyophilize PLUS oligonucleotide. The mixture was incubated overnight at room temperature. Stop Reagent was added to the reaction and incubated at room temperature for 30 minutes. Conjugated antibody was stored in storage solution at $4^{\circ}$.] Cells were washed with wash buffer A $(0.01 \mathrm{M}$ Tris, $0.15 \mathrm{M} \mathrm{NaCl}$ and $0.05 \%$ Tween 20, $\mathrm{pH} 7.4$ ) and incubated with PLA probes (Conjugated probes were diluted 1:200 in Duolink antibody diluent and commercial PLUS and MINUS probes were diluted 1:10 in Duolink antibody diluent) for 1 hour at $37^{\circ} \mathrm{C}$. Cells were washed again with wash buffer $A$ and incubated with amplification mix (amplification stock 1:5 and polymerase $1: 80$ in water) for 100 minutes at $37^{\circ} \mathrm{C}$. Cells were then washed with wash buffer $\mathrm{B}(0.2 \mathrm{M}$ Tris, $0.1 \mathrm{M} \mathrm{NaCl}, \mathrm{pH} 7.5)$ and kept in $1 \mathrm{X}$ PBS before imaging. Negative controls consisted of omission of either of the primary antibodies, while secondary controls were done by omitting all primary antibodies. A list of all the antibodies used in this study is provided in supplementary table 2 . 


\section{$\underline{\text { Image acquisition }}$}

The PLA was imaged using the Zeiss Axio Observer Z1 inverted microscope with a 40X oil objective. The imaging was always done keeping conditions constant for samples from the same experiments. Secondary controls were always imaged using the same conditions as sample sections.

\section{$\underline{\text { Analysis }}$}

Image analysis was done using NIH ImageJ. The background for all conditions was determined as; mean intensity $(\mathrm{AU})+3^{*}$ standard deviation of 3 randomly chosen GFP negative cells per field of view. The highest background value (among all conditions) was used as threshold for all the conditions. GFP positive cells (from Piezo2-GST IRES GFP expression) were chosen for each field of view and the PLA signal was analyzed for these cells, using the "Analyze Particle" tool of NIH ImageJ. The number of PLA puncta, intensity of the PLA signal and total area of positive PLA signal for each cell was measured. The total area of the cell was also measured and the PLA signal values were normalized to total cell area. All values are reported as mean \pm SEM. For statistical analysis, either Mann Whitney test or Kruskal-Wallis test with Dunns's Multiple Comparison Test was used as indicated in the figure legends (GraphPad Prism 6.01, San Diego).

\subsection{Functional characterization of interactors}

\subsubsection{DRG culture and transfection}

The DRG culture protocol was adapted from Coste et al. (2010) and Avenali et al. (2014). 8-9 week old male C57BL/6J mice or Piezo2 ${ }^{\text {GFP }}$ (Woo et al., 2014) were used for preparing primary cultures of dorsal root ganglia neurons. All DRGs were isolated from the mouse and transferred to DMEM/F12 with Glutamax. The tissue was digested with collagenase for 60 minutes followed by papain for 30 minutes at $37^{\circ} \mathrm{C}$. The digested cells were subjected to a BSA column to separate the cells from debris. The cells were then re-suspended in DMEM/F12 Glutamax media supplemented with 10\% horse serum and $100 \mathrm{ng} / \mathrm{ml} \mathrm{NGF}, 50 \mathrm{ng} / \mathrm{ml}$ GDNF, $50 \mathrm{ng} / \mathrm{ml}$ NT-3 and $50 \mathrm{ng} / \mathrm{ml} \mathrm{NT-4}$. Cells were plated on coverslips coated with poly-D-lysine $(1 \mathrm{mg} / \mathrm{mL})$ and laminin $(20 \mu \mathrm{g} / \mathrm{ml})$.

Neurons were transfected with siRNA or plasmid (siRNA: $500 \mathrm{nM}$, plasmids: $0.5 \mu \mathrm{g}$ ), with appropriate controls, using the P3 Primary Cell 4D Nucleofector X Kit; 4D-Nucleofector X Unit according to the manufacturer's instructions (Lonza AG). Transfection was done after the BSA column step and the neurons were allowed to recover for 10 minutes in calcium free RPMI media before being plated. 2 hours after transfection, half of the media 
was exchanged with fresh media containing growth factors. Details of the plasmids and siRNA used in the study are provided in supplementary table 1 and table 3 respectively.

\subsubsection{Quantitative PCR}

Total RNA was isolated from primary cultures of DRG neurons (transfected with candidate siRNA or control siRNA), using the NucleoSpin RNA XS kit according to the manufacturer's instructions. First-strand cDNA synthesis was carried out using QuantiTect reverse transcription kit. Primers for each gene were designed using the Universal ProbeLibrary Assay Design Center (Roche). The list of primers used in this study is provided in supplementary table 4. Gene expression of candidate and Piezo2 were assessed by real-time qPCR using the SYBR green system (Power SYBR Green PCR Master Mix). Samples were input into 386 well plates $(2 \mu \mathrm{L}$ CDNA ( 1-100ng), $100 \mathrm{mM}$ primer (forward and reverse) and $1 \mathrm{X}$ SYBR Green master mix). The measurements were done on a LightCycler 480 instrument (Roche). The melting curve analysis was used to confirm the specificity of the qPCR reaction. All samples were run in triplicate and the negative control reactions were run without template and with the reverse transcription reaction reagents. Threshold cycle $(\mathrm{Ct})$ values, the cycle number in which SYBR green fluorescence rises above background, were normalized to two reference genes ( $\beta$-actin and GAPDH) and recorded as a measure of initial transcript amount. Relative quantification was performed using the 'fit point' and the 'second derivative maximum' method of the LightCycler 480. For statistical analysis, one sample t-test was used (GraphPad Prism 6.01, San Diego).

\subsubsection{Cloning}

Cloning of Pericentrin from mouse tissue

Total RNA was isolated from C57BL/6J mouse brain using the NucleoSpin RNA XS kit. OneTaq One-Step RT-PCR Kit was used to reverse transcribe the RNA into cDNA. The $8.7 \mathrm{~Kb}$ full length construct was amplified as two smaller fragments of $3.9 \mathrm{~Kb}$ and $4.8 \mathrm{~Kb}$. As a template for the cloning, sequence was obtained from NCBI (Nucleotide [Internet]. Bethesda (MD): National Library of Medicine (US), National Center for Biotechnology Information; [1988]. Accession No. NM_008787.3, Mus musculus Pericentrin (kendrin) (Pcnt), transcript variant 1, mRNA; Available from: https://www.ncbi.nlm.nih.gov/nuccore/NM_008787.3). For the amplification PCR, primers were designed to contain EcoRV restriction site at the 5 ' end of the $3.9 \mathrm{~Kb}$ fragment and a Spe1 restriction site at the 3 ' site of the $4.9 \mathrm{~Kb}$ fragment (sequence provided in supplementary table 4). Each fragment was then introduced into a pGEM-T easy vector 
and transformed into library efficiency $\mathrm{DH} 5 \alpha$ cells and grown overnight at $37^{\circ} \mathrm{C}$ on Ampicillin containing plates. From the positively identified clones, the clones without significant mutations were identified using sequencing (compared to MN_08787.3 as described above). Any mutations that were identified were corrected using the Q5 Sitedirected mutagenesis kit. The two fragments were finally cloned into pCMV Sport6, a mammalian expression vector. The expression of the plasmid was verified using immunostainings in HEK293 cells.

\section{Cloning of Mtmr2 C417S enzymatically inactive mutant}

The Mtmr2C417S mutation was first described in Berger et al. (2002) as an catalytically inactivating mutation in mouse Mtmr2. pCMV Mtmr2-myc-DDK construct was used as template to generate the C417S mutant. Q5 site-directed mutagenesis kit was used to introduce a single nucleotide mutation. Primers were designed using the Q5 mutagenesis primer design software (New England Biolabs), and mutagenesis was done according to manufacturer's instruction. A 200bp region was sequenced to ensure that only the desired mutation was introduced. The mutated region was then cloned into the wild-type construct, to avoid the chances of mutations in the other regions of the construct. The expression of the plasmid was verified using immunostaining in HEK293 cells. Details of the primer are provided in supplementary table 4.

\subsubsection{Immunocytochemistry}

\section{Experimental methods}

Coverslips containing HEK293 cells or primary cultures of DRG neurons were washed with $1 \mathrm{X}$ PBS and fixed with 4\% PFA for 10 minutes at room temperature. The cells were then incubated with blocking solution ( $3 \%$ serum, $0.4 \%$ TritonX-100 in 1X PBS) for 30 minutes at room temperature. Following this, the cells were incubated with primary antibody solution (primary antibody diluted in $1 \%$ serum, $0.1 \%$ Triton-100 in $1 \mathrm{X}$ PBS), overnight at $4^{\circ} \mathrm{C}$ with mild shaking. After the overnight incubation, cells were washed with 1X PBS over 15 minutes with gentle shaking. The cells were then incubated with the secondary antibody solution for 2 hours at room temperature. The cells were then washed again with 1 X PBS and mounted with Slowfade Gold antifade reagent with DAPI and allowed to dry before imaging. WGA (Wheat Germ Agglutinin) staining to delineate the neuronal membrane was performed by incubation of neurons with WGA-coupled to Alexa555 for 15 minutes at $37^{\circ} \mathrm{C}$. Cells were then washed three times with neuronal medium, fixed with 4\% PFA for 10 minutes, and subjected to the immunostaining protocol as described above. Antibodies used in this study are listed in supplementary table 2. 


\section{$\underline{\text { Image acquisition }}$}

The slides were imaged using either the Zeiss Axio Observer Z1 inverted microscope or a Zeiss 510 meta confocal laser scanning microscope (CLSM). 10X, 20X air objectives of 40X oil objective was used. The imaging was always done keeping conditions constant for samples from the same experiments. Secondary controls were always imaged using the same conditions as samples.

\section{$\underline{\text { Analysis }}$}

All image analysis was done using NIH ImageJ. To identify the positive cells, threshold was determined as; mean intensity $(\mathrm{AU})+3^{*}$ standard deviation of 10 randomly chosen negative cells. For siRNA experiments, the "cell counter" tool was used to count positive and negative cells in each condition. The "analyze particle tool" from NIH ImageJ was used for quantification of membrane staining intensity. The WGA staining was used to mark the region of interest $(\mathrm{ROI})$ around the cell membrane and for each $\mathrm{ROI}$, the mean intensity of positive signal and mean area of positive signal was determined. For analysis of neuronal regions close to the cells substrate interface, the first five optical sections of each image were used and analysis was done as for the whole cells. For statistical analysis, the Mann Whitney test was used (GraphPad Prism 6.01, San Diego).

\subsubsection{Peptide-peptide/-lipid overlay assay}

Peptide-lipid assay protocol and peptide-peptide overlay assay was adapted from Berger et al. (2002) and Walsh et al. (2012) respectively, with minor modifications. Nitrocellulose membrane or PIP Strips were used for these assays. For the peptide-peptide overlay assay, $0.8 \mu \mathrm{g}$ of peptide (diluted in $\mathrm{dH}_{2} \mathrm{O}$ or $1 \mathrm{X}$ PBS) was spotted on the membrane and allowed to dry for 90 minutes at room temperature, in a humidified chamber. For the peptide-lipid overlay assay, PIP strips were washed once with PBS-T (1X PBS + 1\% Tween-20). The protocol that follows, were common to both assays. The membranes were blocked with $3 \%$ fat-free BSA for 1 hour at room temperature. Membranes were then incubated with $5 \mu \mathrm{g} / \mathrm{mL}$ peptide solution (diluted in 1\% fat-free BSA) for 2 hours at room temperature with gentle shaking. After this, membranes were washed thrice with PBS-T for 7 minutes each and probed with primary antibody (diluted in $1 \%$ fat free BSA) for 2 hours at room temperature. Following which the membranes were washed with PBS-T three times shortly and three times for 5 minutes each. Membranes were then probed with secondary antibody for 1 hour at room temperature and then washed again as described above. Membranes were imaged using the Odyssey Infrared System (LICOR). A list of all peptides used in this study is provided in supplementary table 5. 


\subsubsection{Electrophysiology}

Whole-cell voltage clamp recordings were performed in transfected DRG neurons or HEK293 cells at room temperature. The cells were clamped at $-70 \mathrm{mV}$ for recordings of MA currents. Capacitance compensation was done at $60 \%$ and cells were considered for analysis only if their series resistance was lower than $10 \mathrm{M} \Omega$.

To measure MA currents (protocol adopted from Coste et al. (2010); Figure 9), cells were stimulated mechanically with a blunt glass probe (fire polished borosilicate glass capillary). The stimulation was delivered using a piezo-electrically driven micromanipulator (Physik Instrumente GmBH\&Co. KG). The probe was positioned $4 \mu \mathrm{m}$ away from the cell membrane at the start of the protocol. The probe velocity was $0.8 \mu \mathrm{m} / \mathrm{ms}$ during the ramp phase (forward) and the stimulus duration was set to $150 \mathrm{~ms}$ to allow complete adaptation/inactivation of the channels. The inter-stimulus interval was $180 \mathrm{~ms}$. Stimulus response curves were performed using mechanical stimuli from 0 to $6 \mu \mathrm{m}$ in $1 \mu \mathrm{m}$ increments, and recording elicited currents at a holding potential of $-70 \mathrm{mV}$.

All recordings other than those specifically indicated were made in standard extracellular solution containing (in $\mathrm{mM}$ ) $127 \mathrm{NaCl}, 3 \mathrm{KCl}, 1 \mathrm{MgCl}_{2}, 2.5 \mathrm{CaCl}_{2}, 10$ Glucose and 10 HEPES, $\mathrm{pH}=7.3$, osmolarity $=285 \mathrm{mOsm}$. The intracellular solution for DRG neurons contained (in mM) $133 \mathrm{CsCl}, 10$ HEPES, 5 EGTA, $1 \mathrm{MgCl}_{2}, 1 \mathrm{CaCl}_{2}, 4 \mathrm{MgATP}$ and 0.4 NaGTP, $\mathrm{pH}=7.3$, osmolarity=280 mOsm. Intracellular solution for HEK293 cells contained (in $\mathrm{mM}$ ) $110 \mathrm{KCl}, 10 \mathrm{NaCl}, 1 \mathrm{MgCl}_{2}, 1 \mathrm{EGTA}$ and 10 HEPES, $\mathrm{pH}=7.3$, osmolarity=220 mOsm.

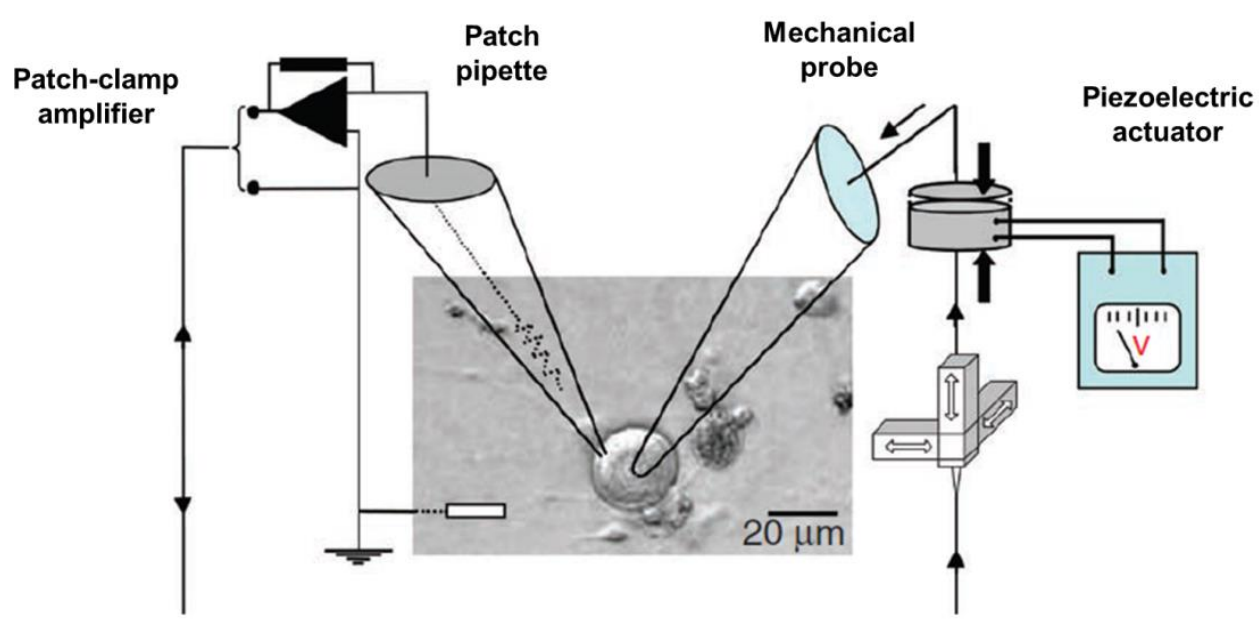

Figure 9: Illustration depicting recording of MA currents

Schematic illustration showing the set-up for recording of MA currents. The cells are plated on poly-D-lysine coated coverslips and cultured for 48-72 hours. During the recording the cells are perfused with extracellular solution. Patch pipette filled with intracellular solution is used to patch the cell and enter whole cell mode (patch pipette shown on the left; holding voltage $-70 \mathrm{mV}$ ). Cells are stimulated using a piezo-electrically driven blunt mechanical probe (shown on the right) to elicit MA currents. (Adapted from Hao and Delmas (2011)) 


\section{Measurement of MA currents under different osmotic condition}

Hypotonic extracellular solution was prepared by diluting regular $\mathrm{CsCl}$ extracellular solution with $\mathrm{ddH}_{2} \mathrm{O}$ to $0.5 \mathrm{X}$ concentration. Based on data from Jia et al. (2016) the following protocol was used for recordings. Cells were incubated with the hypotonic solution for 5 minutes at $37^{\circ} \mathrm{C}$ prior to recording, after which RA-MA currents were recorded for 20 minutes. For osmotically different intracellular solutions, the solutions were introduced in the patch pipette instead of the isotonic intracellular solution and RAMA currents were recorded. Hypertonic intracellular solution (in $\mathrm{mM}$ ): $133 \mathrm{CsCl}, 10$ HEPES, 5 EGTA, $1 \mathrm{MgCl}_{2}, 1 \mathrm{CaCl}_{2}$, $4 \mathrm{MgATP}, 0.4 \mathrm{NaGTP}$ and 120 Sucrose, $\mathrm{pH}=7.3$, osmolarity $=390$ mOsm. Hypotonic intracellular solution (in $\mathrm{mM}$ ): $65 \mathrm{CsCl}, 10 \mathrm{HEPES}, 5$ EGTA, $1 \mathrm{MgCl}_{2}, 1 \mathrm{CaCl}_{2}, 4 \mathrm{MgATP}$ and $0.4 \mathrm{NaGTP} \mathrm{pH}=7.3$, osmolarity=162 mOsm.

\section{$\underline{\text { Pharmacological modulation of MA currents }}$}

During the study, primary cultures of DRG neurons were treated with chemical inhibitors to study the modulation of RA-MA currents. For Nocodazole treatment, cells were incubated with $32 \mu \mathrm{M}$ Nocodazole or vehicle (0.2\% DMSO) (adapted from Jurczyk et al. (2010)) for 30 minutes prior to recording. During the whole-cell recordings of RA-MA currents, $32 \mu \mathrm{M}$ Nocodazole/DMSO was also added to the internal pipette solution. For treatment of DRG neurons with inhibitors of phosphatidylinositol pathway, cells were treated with 35 $\mathrm{M}$ Wortmannin in DMSO (adapted from Mo et al. (2009)) or $1 \mu \mathrm{M}$ Apilimod in DMSO (adapted from Mironova et al. (2016)) for 2 hours prior to recording and recorded using normal extra and intracellular solutions (DMSO only $(0.35 \%$ for Wortmannin and $0.08 \%$ for Apilimod), control was included in all experiments). For phosphatidylinositol phosphate supplementation experiments, $100 \mathrm{nM}, 1 \mu \mathrm{M}$ or $10 \mu \mathrm{M}$ $\mathrm{PI}(3,5) \mathrm{P}_{2}$ or $1 \mu \mathrm{M} \mathrm{PI}(3) \mathrm{P}$ were included in the intracellular $\mathrm{CsCl}$ solution (adapted from Dong et al. (2010)).

\section{$\underline{\text { Analysis }}$}

Analysis protocol was adopted from Coste et al. (2010) and Eijkelkamp et al. (2013). Data analysis and representation was done using using Fitmaster (HEKA Electronik $\mathrm{GmbH}$, Germany) and Igor Pro 6.3 (WaveMetrics). Current magnitude was calculated by measuring peak amplitude (at each stimulus point) and subtracting leak current. To account for variability in cell size, currents were normalized to cell capacitance and represented as current density $(\mathrm{pA} / \mathrm{pF})$. For measurement of inactivation kinetics, current traces reaching at least $75 \%$ of the maximal current amplitude per cell were fitted with mono-exponential or bi-exponential equation and the fast time constant was used for analysis. 
For analysis of Stim $_{50}$, current density was normalized to maximum current to obtain normalized values ranging from 0 to 1 . The data was fit using the sigmoidal equation: $I_{0}+I_{\max } /\left(1+\exp \left(S_{1 / 2}-S\right) /(\right.$ rate $\left.)\right)$, where $I_{0}$ represents minimum current, $I_{\max }$ represents maximum current, $S_{1 / 2}$ represents stimulation value that elicited a current value, $50 \%$ of $I_{\max }$ (as described in Hao et al. (2013)). Values are reported as mean \pm SEM of all analyzed cells. (Only cells that responded to the maximum stimulus magnitude $(6 \mu \mathrm{m})$ were considered for this analysis).

For activation threshold analysis, the stimulus magnitude at which current density crossed $10 \%$ of $I_{\max }$ of control was measured (adapted from Eijkelkamp et al. (2013)). Data are represented as mean \pm SEM.

For statistical analysis, outlier analysis was carried out using Grubb's t-test followed by verification using mean $+3^{*}$ standard deviation criterion. Only if a value was detected as an outlier by both these (Grubb's outlier and >mean+3*standard deviation) tests it was considered a bona fide outlier and removed from the data set. For analysis of stimuluscurrent curves, 2-way ANOVA with Holm-Sidak's post hoc test was employed. For analysis of activation threshold, Stim $_{50}$ and inactivation time, Mann Whitney test or 1-way ANOVA (Kruskal-Wallis test with Dunn's Multiple Comparison test) was used. Chi square test was used for the analysis of population distribution of MA currents (GraphPad Prism 6.01, San Diego).

It is important to note that current magnitudes and activation thresholds cannot be compared between experiments because recordings of MA currents were done on different days in vitro (DIV) and cells were subjected to different treatments, transfections etc. Keeping this in mind each experiment was performed with its appropriate controls.

\subsubsection{Behavioral testing}

Behavioral testing was performed by Meike Hütte (Somatosensory signaling and Systems biology group, Max Planck Institute for Experimental medicine). 9-10 week old C57BL/6 mice were used for these experiments. The experiments were carried out in compliance with institutional guidelines of the Max Planck Society and guidelines of the Landesamt für Verbraucherschutz und Lebensmittelsicherheit of Lower Saxony, Germany (AZ 33.9-42502-04-14/1638). The animals were acclimatized for 2 hours in the testing device (Plantar Aesthesiometer, Ugo Basile) and then injected subcutaneously into the midplanter region of the left hind paw. $10 \mu \mathrm{L}$ of the respective experimental agent was injected.

Experimental agents used: Apilimod (Cayman Chemical; stock solution: $20 \mu \mathrm{g} / \mu \mathrm{L}$ dissolved in DMSO; diluted 1:100 in 1X PBS to a working concentration of $0.2 \mu \mathrm{g} / \mu \mathrm{L}$ ), vehicle (1\% DMSO in $1 \mathrm{X}$ PBS), hypo osmotic solution (0.5X PBS, osmolarity= 155 
mOsm; Of note, 1X PBS, osmolarity=270-300 mOsm (ThermoFisher Scientific)), Apilimod

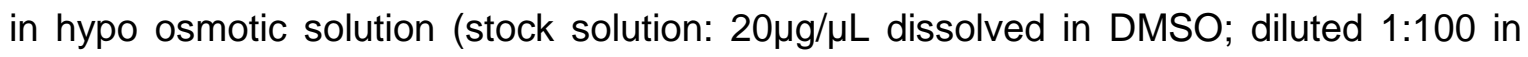
$0.5 X$ PBS (155 mOsm) to a working concentration of $0.2 \mu \mathrm{g} / \mu \mathrm{L}$ ).

Response to innocuous mechanical stimulation, was measured using a Plantar Aesthesiometer (Ugo Basile) by applying a graded force (0-10 $\mathrm{g}$ in 40 seconds) to the plantar surface of the hind paw. Measurements were made between 0-90 minutes after the injection. Three independent measurements were made per paw and an average of these values was used to calculate withdrawal thresholds (force, g) and withdrawal latencies (time, seconds). Comparison was always made between the ipsilateral (injected) paw and the contralateral (un-injected) paw. This accounted for changes in the animal's arousal and stress caused by the unilateral injection. Three independent mouse cohorts were used for the experiments.

Statistics

Data was analyzed using GraphPad Prism 6.01. For analysis of reduction in withdrawal threshold and average withdrawal threshold, 2-way ANOVA followed by Holm-Sidak's post hoc tests were used. 


\section{RESULTS: Part 1}

A large part of the results in this section are based on Narayanan et al. (2016). Reprinted (adapted) with permission from Narayanan et al. (2016). Copyright (2016) American Chemical Society.

\subsection{Verification of Piezo2-Pericentrin (Pcnt) interaction as proof of principle}

Since the identification of Piezo2, its function and modulation have been of great interest. In order to systematically identify the interactors of Piezo2, in 2016 our lab reported an affinity purification based approach to identify novel interactors of native Piezo2 from DRG neurons in mice (Narayanan et al., 2016). Figure 5 illustrates the outline of the experimental approach used towards this goal. Through this screen, 36 potential interactors of mouse Piezo2 in DRG neurons were identified. Figure 6 summarizes the results obtained from this part of the study (Narayanan et al., 2016). After the identification of potential interactors, the first step was to assess the robustness of the screen using orthogonal methods. For this purpose a candidate protein, identified on our screen as significant ( $p=0.022$, unpaired $t$-test) but low score interactor was chosen. It exhibited a moderate fold change (3.6-fold enriched compared to control) and represented a highly unlikely interactor of Piezo2, namely Pericentrin (Pcnt). Pcnt is expressed across tissues and cells and is known for its centrosomal localization and role in cell-cycle regulation and microtubule organization (Delaval and Doxsey, 2010). Additional roles for Pcnt have been described in the visual and olfactory system (EndohYamagami et al., 2010; Miyoshi et al., 2009; Mühlhans et al., 2011). Interestingly, Pcnt is linked to mechanotransduction in the Drosophila mechanosensory system. Loss of Pcnt causes mechanosensory deficits in the fly, through malformation of sensory cilia (Martinez-Campos et al., 2004).

\subsubsection{Piezo2 and Pericentrin (Pcnt) co-immunoprecipitate, in HEK293 cells}

To validate the mass-spectrometry results, co-immunoprecipitation was done as described in the methods section (2.1.2). I co-expressed Piezo2-GST and Pcnt in HEK293 cells. Anti-GST antibody was used to pull down Piezo2 from the cells and the elute (IP) and cell lysate fractions were resolved using gel electrophoresis and western blotting. The blots were probed with anti-Pcnt antibody and anti-GST antibody. Pcnt was identified in the elute fraction when overexpressed with Piezo2-GST but not with mock plasmid (Figure 10A). Importantly, anti-Pcnt antibody identified Pcnt in the lysate of Piezo2+Pcnt and Mock+Pcnt. This indicates that a) Pcnt can be successfully 
overexpressed in HEK293 cells and b) when co-expressed with Piezo2, it forms an association with the channel and can be co-immunoprecipitated with it. Figure 10B illustrates that Piezo2-GST was also identified in the elute fraction (IP) when Pcnt and Piezo2 are co-expressed. Lack of Piezo2 signal in the cell lysate could be due to the higher dilution of the lysate and/or low expression of Piezo2. Of note, the anti-Pcnt antibody was validated through siRNA mediated knockdown experiments before being used for the immunoblotting (data in following sections).

A

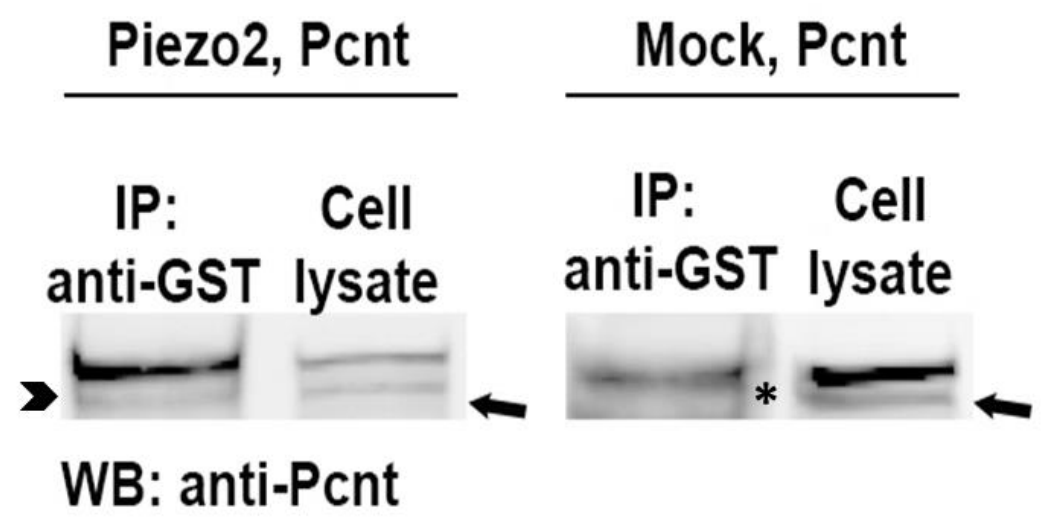

B
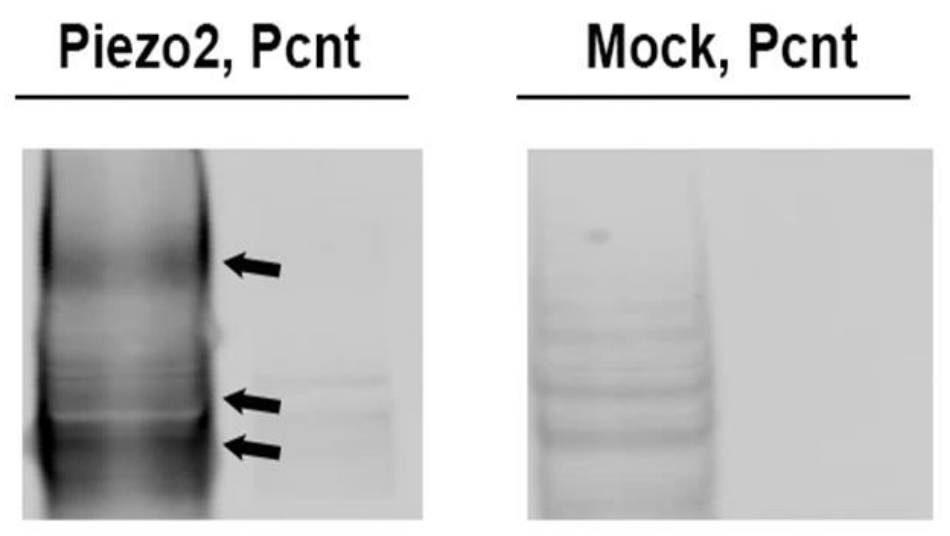

\section{WB: anti-GST}

Figure 10: Co-immunoprecipitation (IP) of Piezo2 and Pericentrin in HEK293 cells

Representative immunoblots of Piezo2-GST and Pcnt co-immunoprecipitation in HEK293 cells. (A) anti-GST antibody was used to pulldown Piezo2-GST from HEK293 cells. Piezo2 was co-expressed with either Pcnt or Mock plasmid. Upon co-expression with Pericentrin, anti-Pcnt antibody detects Pcnt in the IP (immunopurified product; arrowhead) indicating that Pcnt is pulled down with Piezo2. In case of coexpression with mock, however, Pcnt can only be identified in the cell-lysate (arrows) but not in the immunopurified product (IP; *)). (B) Piezo2 is observed in elute (IP) of HEK293 cells expressing Piezo2 and Pericentrin (arrows) but not in Mock+Pcnt. This indicates that Piezo2-GST is expressed in HEK293 cells and can be immunoprecipitated with the anti-GST antibody. Blots were repeated at least three times. Reprinted (adapted) with permission from Narayanan et al. (2016). Copyright (2016) American Chemical Society. 


\subsubsection{Pericentrin (Pcnt) is expressed in the somatosensory system}

\section{Pericentrin is expressed in peripheral nerve endings}

In order to characterize Pcnt expression in the somatosensory system, I used tissue from Piezo2 ${ }^{\text {GFP }}$ mice which expresses Piezo2 tagged with a C-terminal GFP (Woo et al., 2014). This allows for immunolabelling of Piezo2 using the anti-GFP antibody. Of note, the Piezo2 antibody that was tested positive during the mass-spectrometry experiments (section 1.3.4) did not work well in the immunohistochemistry experiments. In order to gain reliable insights into the localization of Piezo2, the GFP immunolabelling approach was used instead.

It is known that Piezo2 is expressed in LTMRs in the backskin of mice (Ikeda et al., 2014; Maksimovic et al., 2014; Ranade et al., 2014; Schrenk-Siemens et al., 2014; Woo et al., 2014). Based on this, expression of Pcnt was analyzed in the same structures. Indeed, in cross-sections of the back hairy skin from mice, I observed Pcnt staining in nerve fibers innervating hair follicles. The staining was observed in both lanceolate (arrows in Figure 11A) and circumferential endings (arrowheads in Figure 11A) (Abraira and Ginty, 2013; Li and Ginty, 2014; Shenton et al., 2014). Interestingly, some of the Pcnt label colocalized with the Piezo2-GFP staining while some of it appeared to be in closed apposition with Piezo2. Co-staining with Tuj1 (neuronal marker), also showed that Pcnt is expressed in neuronal structures or at least in close apposition with them (Figure 11A, upper panel). Additionally, lower panel of Figure 11A shows that Pcnt immunolabel also appears to be in close apposition with the Schwann cell marker, S100 (Li and Ginty, 2014; Shenton et al., 2014) immunolabel. This novel localization of Pcnt is interesting because so far Pcnt has only been reported in cell bodies and primary cilia (Delaval and Doxsey, 2010). A major limitation of these experiments arises from the structure of these terminals. The nerve endings innervating the hair follicle are tightly engulfed by terminal Schwann cells. Thus, resolution of conventional light microscopy proved insufficient to localize Pcnt precisely in these structures (Li and Ginty, 2014; Shenton et al., 2014). 
A

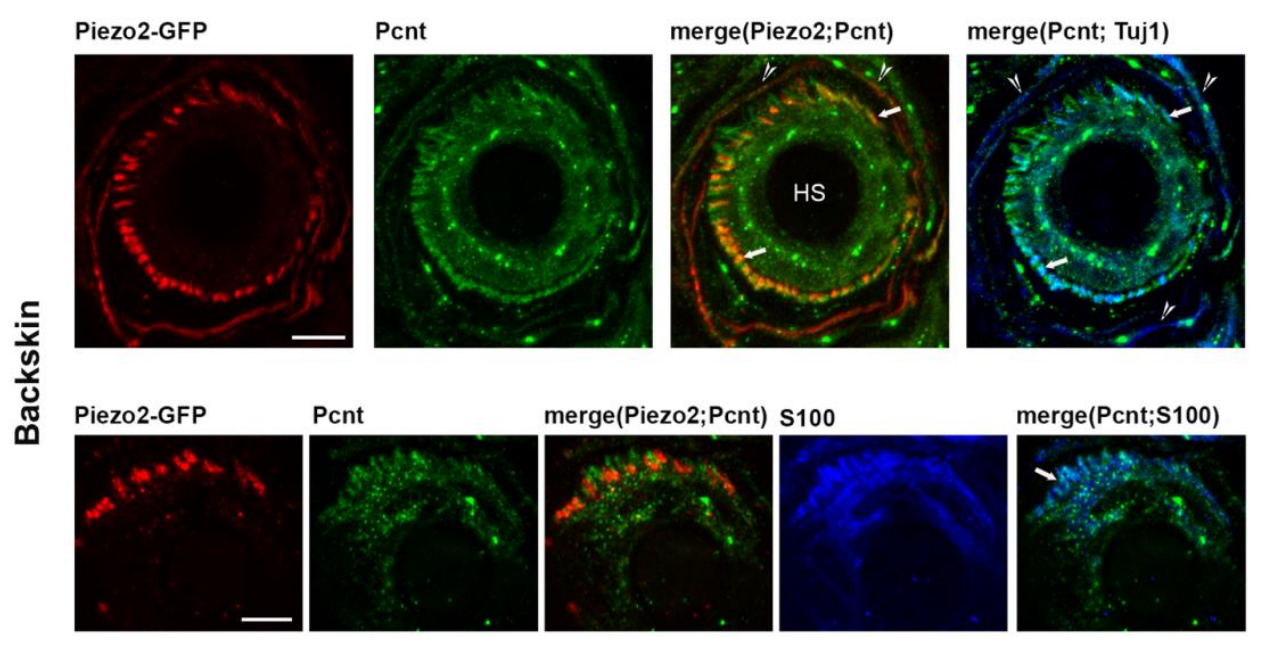

B
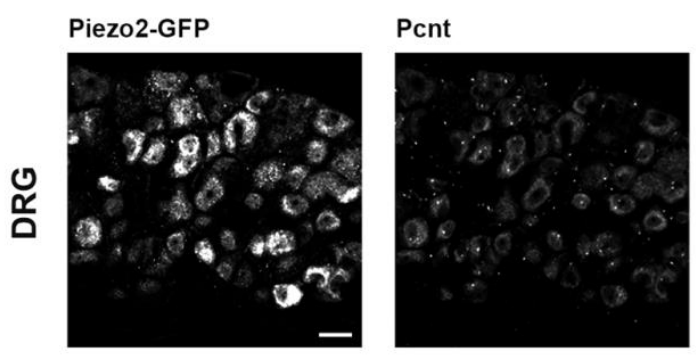

merge

C

\section{D}
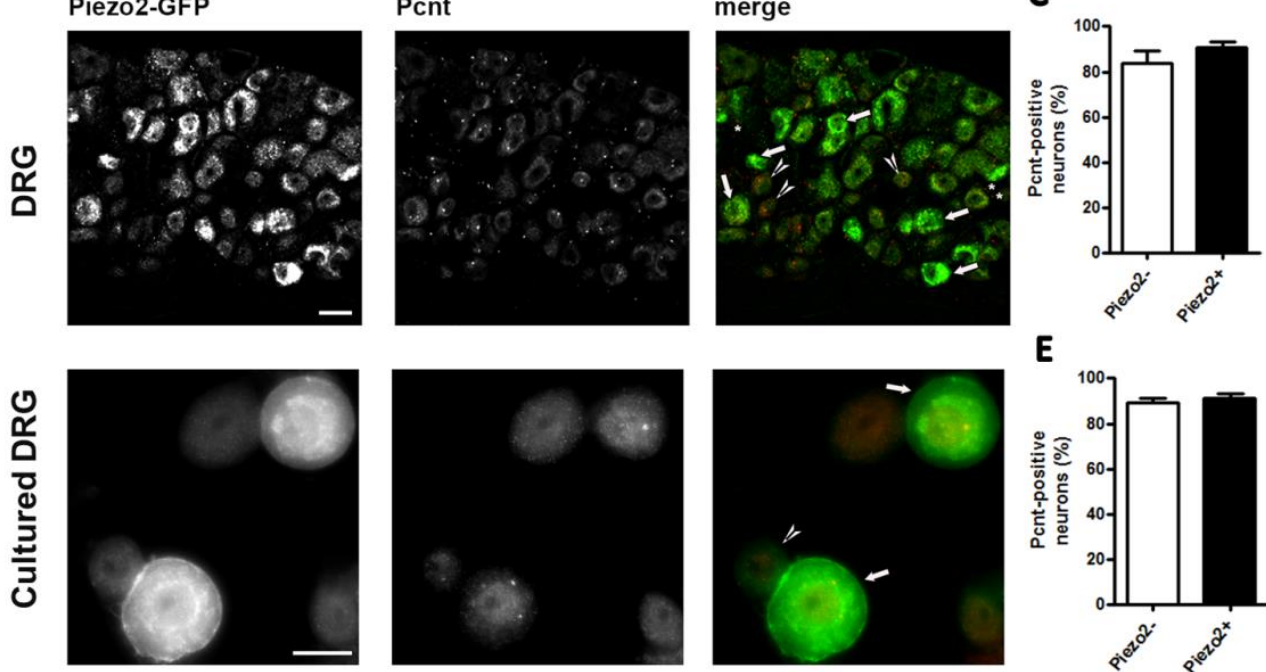

E
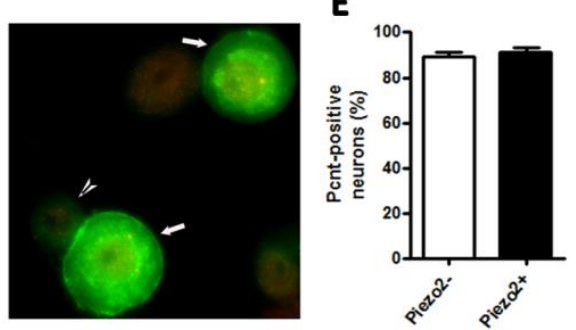

Figure 11: Pericentrin expression in the mammalian somatosensory system

(A) Upper and lower panel show representative immunohistochemistry images from the cross section of back skin from Piezo2 ${ }^{G F P}$ mice showing the innervation of hair follicle by Piezo2 positive fibers. Piezo2-GFP is identified in the lanceolate (arrows) and circumferential endings (arrowheads) that innervate the hair follicles. Immunolabelling against Pcnt shows significant overlap or apposition with the Piezo2-GFP staining. Pcnt also shows considerable overlap/apposition with Tuj1 (neuronal marker). Bottom panel shows magnified images of the upper panel showing the close apposition of Pcnt with Piezo2-GFP and the S100 immunolabel in lanceolate endings (arrow); $\mathrm{N}>3$ for each immunolabel. (B) Representative immunohistochemistry and (D) immunocytochemistry of Pcnt immunolabel in cell bodies of dorsal root ganglia (DRG neurons) in tissue and primary culture (from Piezo2 ${ }^{G F P}$ mice) respectively. Pcnt is seen as a centrosomal puncta as well as dispersed staining in the cytoplasm of Piezo2 positive (arrows) and Piezo2 negative neurons (arrowheads). (C) and (E) quantification of Pcnt immunolabel in Piezo2 positive and Piezo2 negative neurons in DRG tissue and primary culture, respectively. Pcnt immunolabel shows no significant differences between the two populations of neurons (Immunohistochemistry: Pcnt in Piezo2 positive cells: $91.5 \pm 2.36 \%$; Pcnt in Piezo2 negative cells: $84.6 \pm 5.37 \%$; $n>2000$ neurons; $n s ;$ unpaired ttest; Immunocytochemistry: Pcnt in Piezo2 positive cells: $91.0 \pm 2.38 \%$; Pcnt in Piezo2 negative cells: $84.0 \pm$

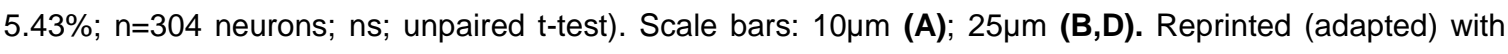
permission from Narayanan et al. (2016). Copyright (2016) American Chemical Society. ${ }^{*}: p<0.05$; ${ }^{* *}$ : $p<0.01 ;{ }^{* * *}: p<0.0001 ;{ }^{* \star * *}: p<0.00001$; data are not significant unless otherwise stated. 


\section{Pericentrin is expressed in cell bodies of DRG neurons}

Due to the difficulties of resolving the structural details in the peripheral nerve endings, I tested the anti-Pcnt antibody in cell bodies of DRG neurons. Two commercial Pcnt antibodies were tested, which have been used extensively in literature (Griffith et al., 2008; Miyoshi et al., 2006; Miyoshi et al., 2009; Mühlhans et al., 2011; Mühlhans and Gießl, 2012; Zu et al., 2015).

Consistent with the above mentioned studies, Pcnt was found as a distinct centrosomal puncta as well as smaller puncta throughout the cytoplasm. Figure 11B shows representative images of Pcnt and GFP staining in DRG tissue from Piezo2 ${ }^{\text {GFP }}$ mice. In order to test if Pcnt distribution in the DRG neurons was related to Piezo2 positivity of the cells, the number of Pcnt positive neurons in Piezo2 positive and Piezo2 negative populations were counted. I found no significant difference in the Pcnt expressing cells among the two populations (unpaired t-test; Figure 11C).

Similar results were obtained when DRG cultures prepared from the Piezo2 ${ }^{\text {GFP }}$ mice were analyzed (unpaired t-test; Figure 11D and E). Interestingly, this data is supported by a recent study using RNA-seq analysis of DRG neurons. The study showed that Pcnt is ubiquitously expressed in the different DRG neuron populations (Usoskin et al., 2015).

\section{$\underline{\text { Piezo2 and Pericentrin are expressed in close proximity in DRG neurons }}$}

After the identification of Pcnt in the somatosensory system, the next step was to study its expression in the cell in relation to Piezo2. Towards this aim, proximity ligation assay (PLA) was used (Söderberg et al., 2006). In PLA, primary antibodies are used to detect the proteins of interest which are then probed by a secondary antibody coupled with oligonucleotide strands. If the two proteins are at distance, maximum of $40 \mathrm{~nm}$ apart, the oligonucleotides participate in a rolling circle DNA synthesis which amplifies the signal several hundred-fold. This signal can then be detected by fluorescently labelled complimentary oligonucleotide probes. The result is a distinct fluorescent puncta indicating a point of interaction of the two proteins which can be visualized with fluorescence microscopy. In DRG neurons, this technique has been successfully used to demonstrate TRPV1-GABA ${ }_{B}$ interaction (Hanack et al., 2015).

To test proximity of Piezo2 and Pcnt, DRG cultures prepared from Piezo2 ${ }^{\text {GFP }}$ mice were used. Primary antibodies against GFP and Pcnt were used to detect the proteins of interest. As mentioned in the previous section, the anti-Piezo2 antibody was found to be unreliable in immunostainings and hence was not used for these experiments. Using antiGFP and anti-Pcnt antibodies, a robust PLA signal was observed (Figure 12A) while the no specific signal was observed upon omission of either antibody (results shown for omission of anti-Pcnt antibody).The number of PLA puncta/neuron was analyzed to 
provide a quantitative measure of the assay. The number of puncta/neuron was significantly higher when both antibodies were used compared to omission of either antibody (Mann Whitney test; Figure 12B). These results indicate that Pcnt and Piezo2GFP are indeed localized in close proximity to each other (maximally 40nm apart) in DRG neurons. These results prompted the question; what does this proximity of Piezo2 and Pcnt mean for the function of the channel?

A

CTRL

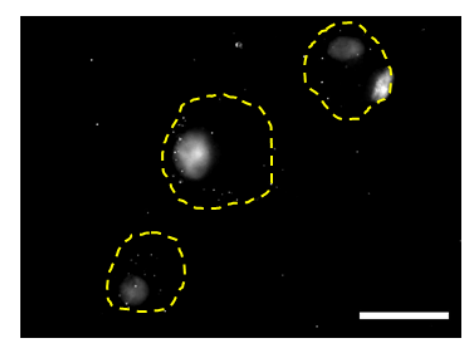

Piezo2-GFP + Pcnt

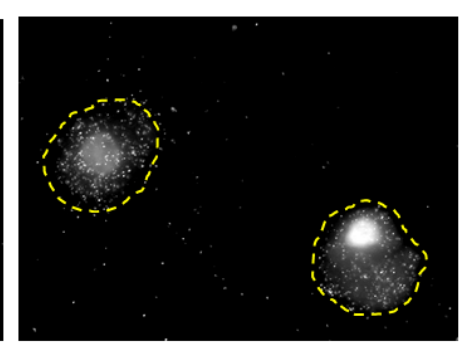

B

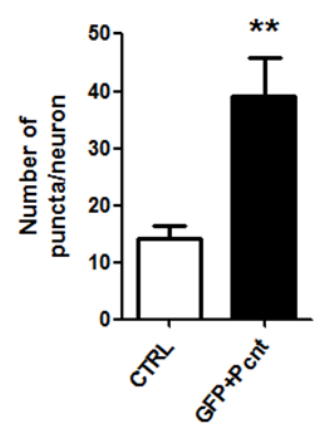

Figure 12: Piezo2 and Pericentrin express in close proximity in DRG neurons

Representative images (A) and quantification (B) of proximity ligation assay (PLA) in DRG neurons cultured from Piezo2 $2^{G F P}$ mice. Anti-GFP and anti-Pcnt antibodies were used to detect Piezo2 and Pericentrin respectively. Robust PLA signal is seen when both antibodies are used, while omission of either antibody (CTRL; shown here for omission of anti-Pcnt antibody) results in a significant decrease of the PLA signal (anti-GFP + anti-Pcnt: $39 \pm 7$ puncta/neuron; only anti-GFP: $14 \pm 2$ puncta/neuron; $n=32-65$ neurons per condition; $p=0.0012$; Mann Whitney test; the number of punta were rounded off to the nearest whole number). Scale bar $=25 \mu \mathrm{m}$. Reprinted (adapted) with permission from Narayanan et al. (2016). Copyright (2016) American Chemical Society. ${ }^{*}: p<0.05 ;{ }^{* *}: p<0.01 ;{ }^{* * *}: p<0.0001 ;{ }^{* * *}: p<0.00001$; data are not significant unless otherwise stated.

\subsection{Functional characterization of Piezo2-Pericentrin (Pcnt) interaction in DRG neurons}

In order to understand how Pent may influence Piezo2 channel function, I used DRG cultures from mice as the model system. The DRG cultures provide an ideal system for targeted manipulation of protein levels and assessment of Piezo2 function. Of note, Piezo2 was first described in the DRG cultures system (Coste et al., 2010).

\section{Pericentrin siRNA decreases Pcnt mRNA and protein levels in DRG neurons}

To study the relevance of Pcnt for Piezo2 function, I used nucleofection to transfect DRG neurons with siRNA against Pcnt. As control, non-targetting siRNA (CTRL) was transfected. The details of the transfection are described in the methods section (2.2.1). Cells were maintained in culture for 72 hours prior to analysis. In order to quantify the 
knockdown of Pcnt upon siRNA transfection in DRG neurons, real-time quantitative PCR was used as described in the methods section (2.2.2). Pcnt mRNA was significantly reduced in Pcnt siRNA transfected cells (Pcnt siRNA) compared to control transfected cells (CTRL). This was measured relative to two housekeeping genes $\beta$-actin and GAPDH (one sample t-test; Figure 13A). To check if Piezo2 is changed upon Pcnt knockdown, Piezo2 mRNA was also measured in these cultures. No significant difference was observed in Piezo2 mRNA upon Pcnt knockdown (one sample t-test; Figure 13B). The results show that Pcnt siRNA was successful in knocking down Pcnt in DRG neurons without affecting Piezo2 mRNA levels.

Besides mRNA levels, I also quantified protein levels of Pcnt upon knockdown using immunostaining. Figure $13 \mathrm{C}$ shows representative immunostaining of Pcnt in DRG neurons upon transfection with non-targeting siRNA (CTRL) or Pcnt siRNA. The number of Pcnt positive cells was found to be significantly decreased upon siRNA knockdown of Pcnt (unpaired t-test; Figure 13D). Importantly, in Pcnt negative cells, both centrosomal and cytoplasmic Pcnt staining was either entirely absent or significantly reduced in intensity compared to Pcnt positive cells. The results, besides confirming that Pcnt siRNA successfully decreases Pcnt protein expression also confirm the specificity of the Pcnt antibodies (results were comparable for both Pcnt antibodies tested; Abcam and Covance).

\section{Figure 13: Knockdown of Pericentrin in DRG neurons}

Quantification of Pcnt mRNA under control (CTRL) and Pcnt siRNA condition (A) shows a significant decrease in the Pcnt mRNA under siRNA condition compared to CTRL (shown for actin as reference housekeeping gene (Pcnt siRNA, actin as reference: $0.31 \pm 0.11 ; N=5$ cultures; $p=0.0036$; one sample t-test (compared to a theoretical mean of $1.00(C T R L)$ ). Of note, similar results were obtained when using GAPDH as reference (data not shown). Quantification of Piezo2 mRNA upon Pcnt knockdown (B) shows no significant difference between CTRL and Pcnt siRNA (Pcnt siRNA, actin as reference: $1.14 \pm 0.22 ; \mathrm{N}=5$ cultures; ns; one sample t-test (compared to a theoretical mean of 1.00 (CTRL)). Similar results were obtained when using GAPDH as reference (data not shown). Representative images (C) and quantification (D) of the Pcnt immunolabel in DRG neurons upon Pcnt knockdown. Pcnt signal was significantly reduced from both centrosomes and cytoplasm, upon treatment with Pcnt siRNA (Pcnt positive cells in CTRL: 92.70 $\pm 0.94 \%$; Pcnt positive cells in Pcnt siRNA: $47.60 \pm 2.87 \%$; $N=14$ cultures and $n>1000$ neurons; $p<0.0001$; unpaired t-test). Scale bar=15 $\mu \mathrm{m}$. Reprinted (adapted) with permission from Narayanan et al. (2016). Copyright (2016) American Chemical Society. ${ }^{*}: p<0.05 ;{ }^{* *}: p<0.01 ;{ }^{* *}: p<0.0001 ;{ }^{* * *}: p<0.00001$; data are not significant unless otherwise stated. 
A

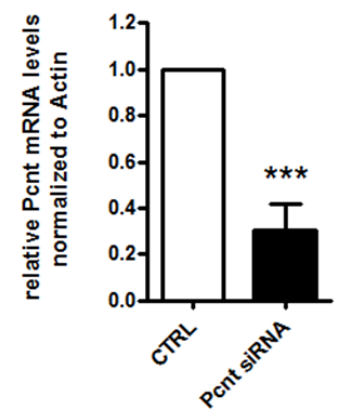

C

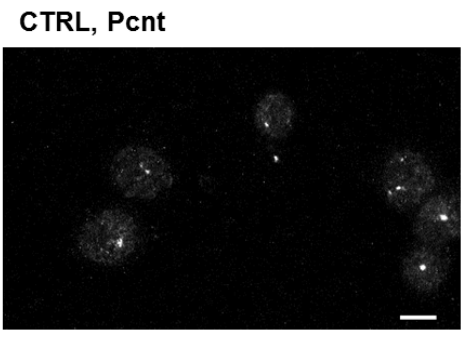

Pcnt siRnA, Pcnt

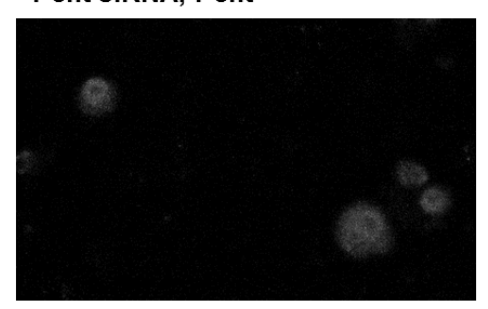

B

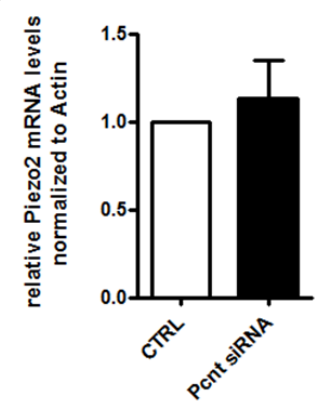

D

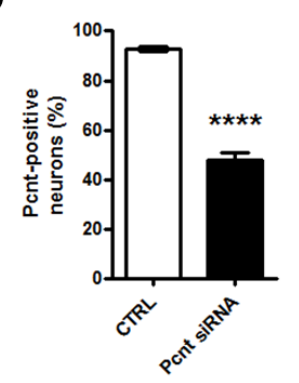

\subsubsection{Pericentrin (Pcnt) knockdown potentiates Piezo2 mediated MA currents}

To study the effect of Pcnt knockdown in DRG neurons on Piezo2, the most direct method is to measure Piezo2 mediated RA-MA currents. As described in the methods section (2.2.6), whole cell patch-clamp coupled with mechanical stimulation was used to measure Piezo2 MA currents.

In brief, large diameter DRG neurons were stimulated with a blunt glass probe while whole cell MA currents were recorded (as sown in Figure 9). Large diameter neurons were chosen because these are known to exhibit Piezo2 mediated currents (Coste et al., 2010). MA currents were measured 72 hours after siRNA transfection. Figure 14A shows representative current traces obtained from CTRL or Pcnt siRNA treated neurons. As can be clearly seen, upon Pcnt knockdown, there is a significant increase in the Piezo2 mediated RA-MA.

This is quantified in the stimulus-current curves (2-way ANOVA and post hoc Bonferroni test; Figure 14B). I also measured the activation threshold of the whole cell RA-MA currents upon Pcnt knockdown. This is defined as the stimulus required to elicit a minimum discernable current (in our case defined at $10 \%$ of Imax of CTRL currents). The activation threshold of Pcnt siRNA treated cells was significantly reduced, which correlates with the increased current magnitude (unpaired t-test; Figure 14C). Additionally, another feature of RA-MA currents is the Stim $_{50}$ (stimulus required to elicit $50 \%$ of maximum current). This property remained unchanged upon Pcnt knockdown (unpaired t-test; Figure 14D). I also analyzed the inactivation time constant ( $\mathrm{T}$ ) of the RA- 
MA currents and found no significant differences between CTRL and Pcnt siRNA (unpaired t-test; Figure 14E).

The data indicate that loss of Pcnt potentiates Piezo2 mediated RA-MA currents. Importantly, the defining property of the RA-MA currents (namely the rapid inactivation) remains unaffected upon Pcnt knockdown.

A

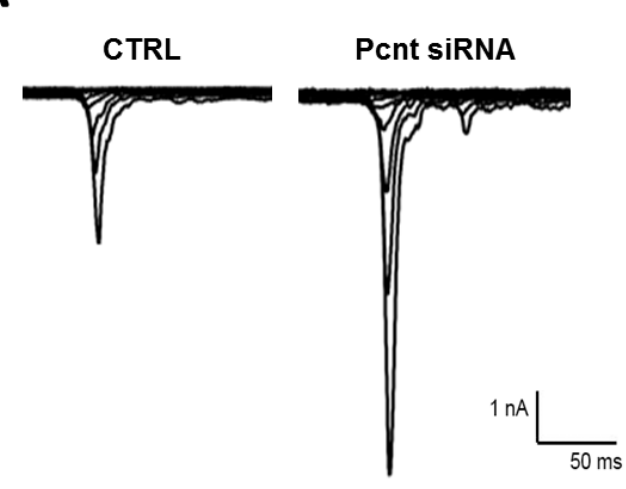

C

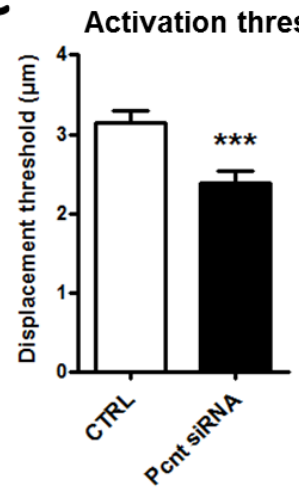

B

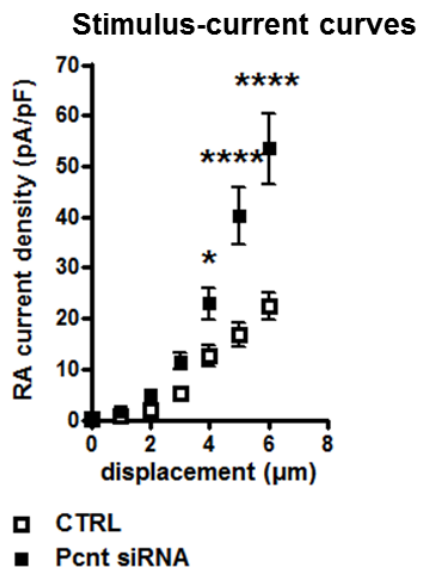

$\mathbf{E}$

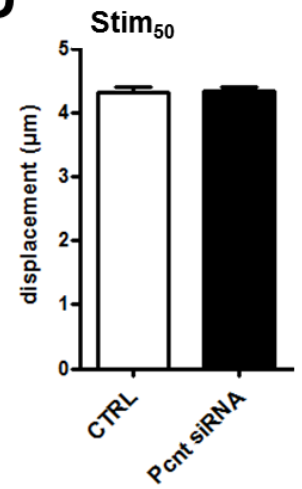

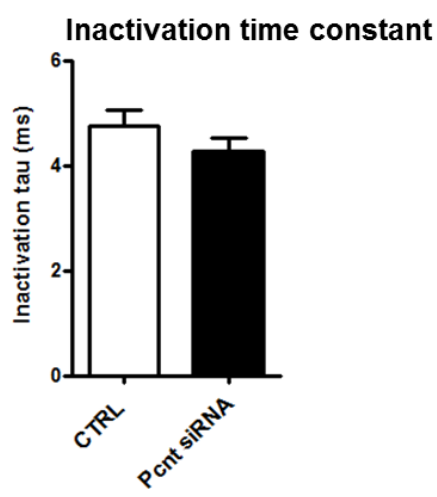

Figure 14: Pericentrin knockdown in DRG neurons potentiates RA-MA currents

Representative traces of RA-MA currents in CTRL and Pcnt siRNA treated DRG neurons (A). Stimuluscurrent curves (B) show that Pcnt knockdown causes significant increase in the Piezo2 mediated RA-MA currents ( $\mathrm{n}=50-52$ neurons per conditions; 2-way ANOVA and post hoc Bonferroni test). Activation threshold (C) of RA-MA currents upon loss of Pericentrin is significantly lower than CTRL (CTRL: $4.09 \pm 0.15 \mu \mathrm{m}$; Pcnt siRNA: $3.10 \pm 0.15 \mu \mathrm{m} ; \mathrm{n}=50-52$ neurons per condition; $\mathrm{p}<0.0001$; unpaired t-test). Stim 50 of RA-MA currents (D) remains unchanged upon Pcnt knockdown (CTRL: $4.32 \pm 0.08 \mu \mathrm{m}$; Pcnt siRNA: $4.32 \pm 0.08 \mu \mathrm{m}$; $n=50-52$ neurons per condition; ns; unpaired t-test). Inactivation time constant $(\tau)$ of RA-MA currents (E) remains unchanged upon Pcnt knockdown (CTRL: $4.78 \pm 0.28 \mathrm{~ms}$; Pcnt siRNA: $4.29 \pm 0.25 \mathrm{~ms}$; $n=50-52$ neurons per condition; ns; unpaired t-test). Reprinted (adapted) with permission from Narayanan et al. (2016). Copyright (2016) American Chemical Society. *: $p<0.05$; ${ }^{* *}: p<0.01 ;{ }^{* \star *}: p<0.0001 ;{ }^{* \star * *}: p<0.00001$; data are not significant unless otherwise stated. 


\subsubsection{Pericentrin (Pcnt) knockdown does not affect other MA currents or population of MA currents}

As mentioned in the introduction, DRG neurons in mice exhibit three types of MA currents which can be distinguished based on their inactivation time constants ( $\tau$ ) (Coste et al., 2010). The RA-MA currents are predominantly mediated by Piezo2 (Coste et al., 2010; Dubin et al., 2012; Ranade et al., 2014). While little is known about IA-MA currents, SAMA currents were recently shown to be mediated by Tentonin 3/TMEM150c (Hong et al., 2016). In order to know if Pcnt knockdown affected other MA currents in DRG neurons, I recorded IA and SA-MA currents from neurons treated with Pcnt siRNA or CTRL. I found that neither IA-MA nor SA-MA currents were affected by Pcnt knockdown (Figure 15A and B). The stimulus-current curves show no differences in magnitude between conditions (2-way ANOVA and post hoc Bonferroni test; Figure 15A and B). The activation threshold of the IA-MA and SA-MA currents was also unchanged upon Pcnt knockdown, indicating that the influence of Pcnt was remarkably specific to Piezo2 RAMA currents (Activation threshold: IA-MA: CTRL: $2.47 \pm 0.33 \mu \mathrm{m}$; Pcnt siRNA: $2.16 \pm$ $0.20 \mu \mathrm{m}$; ns; 17-18 neurons per condition; unpaired t-test; SA-MA: CTRL: $1.50 \pm 0.50 \mu \mathrm{m}$; Pcnt siRNA: $1.28 \pm 0.16 \mu \mathrm{m}$; ns; 7-8 neurons analyzed per condition; unpaired t-test).

As mentioned above there are different types of MA currents in DRG neurons and each current type is exhibited by defined populations in the system. Considering, loss of Pcnt potentiates RA-MA currents; I wanted to check if the number of cells exhibiting these currents may also have been changed. As shown in Figure 15C, I did not observe any significant differences in the fractions of different MA current populations in cultures treated with CTRL of Pcnt siRNA (Fisher's exact test; Figure 15C). The data is consistent with previously reported proportions of MA currents in DRG cultures (Coste et al., 2010). Therefore, it can be concluded that Pcnt knockdown exerts a very specific effect on Piezo2 RA-MA currents. It neither influences other MA currents nor the general properties of the culture system. 
A

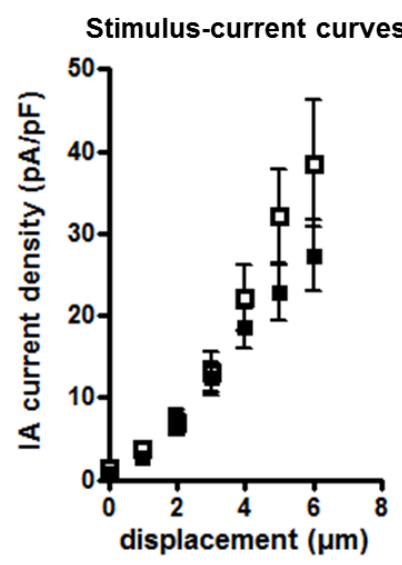

- CTRL

- Pcnt siRnA
B

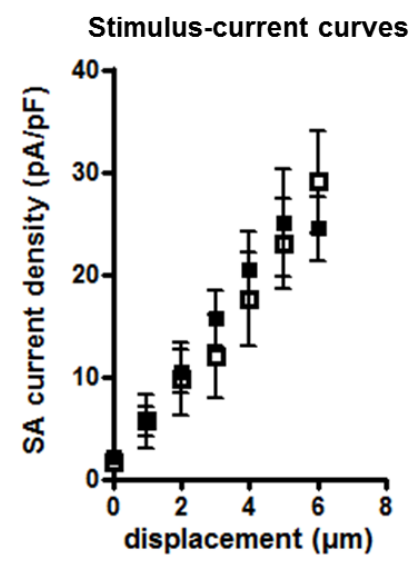

- CTRL

- Pcnt siRnA

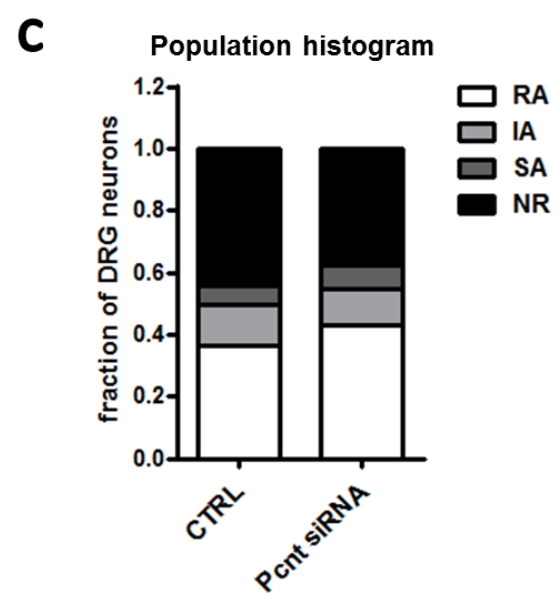

Figure 15: Pericentrin knockdown does not affect IA-MA and SA-MA currents

Stimulus-current curves of IA (A) and SA (B)-MA currents under CTRL or Pcnt siRNA conditions show no significant differences (IA-MA: $n=17-18$ neurons per condition; ns; 2-way ANOVA with post hoc Bonferroni test; SA-MA: $n=7-8$ neurons per condition; ns; 2-way ANOVA with post hoc Bonferroni test). Population distribution of RA-MA, IA-MA, SA-MA and NR (non-responder) neurons in DRG cultures (C) treated with CTRL or Pcnt siRNA. The fraction of cells displaying the different mechanically activated currents remains unchanged upon Pcnt knockdown (RA:IA:SA:NR; CTRL: 0.37: 0.13: 0.06: 0.44; Pcnt siRNA: 0.43: 0.12: 0.08: 0.38; n>200 neurons per condition, pooled data from Figure 14B, Figure 15A and B; ns; Fisher's exact test). Reprinted (adapted) with permission from Narayanan et al. (2016). Copyright (2016) American Chemical Society. ${ }^{*}: p<0.05 ;{ }^{* *}: p<0.01 ;{ }^{* * *}: p<0.0001 ;{ }^{* * *}: p<0.00001$; data are not significant unless otherwise stated. 


\subsubsection{Pericentrin (Pent) overexpression in DRG neurons but not in HEK293 cells affects cell health}

Considering that the loss of Pcnt, potentiated Piezo2 RA-MA currents, the question arose whether overexpression of Pcnt would have the opposite effect. Unfortunately, in contrast to the siRNA transfected DRG neurons, neurons overexpressing Pcnt were severely compromised in terms of health. I observed significant blebbing in the Pcnt transfected neurons and also it was considerably more difficult to record MA currents from these neurons.

Despite these issues, I recorded RA-MA currents from the few healthy cells, 48 hours after transfection. I found a significant reduction in the RA-MA currents, upon overexpression of Pcnt compared to overexpression of empty vector (2-way ANOVA and post hoc Bonferroni test; Figure 16A). The result is in line with what is expected based on the data (Figure 14B) obtained from the knockdown of Pcnt, but due to the compromised cell health, I refrain from interpreting these results.

Since the cellular health was compromised by the overexpression of Pcnt in DRG neurons, I tested if overexpression in heterologous system could be used to study the Piezo2-Pcnt interaction. Piezo2 was co-expressed with mock plasmid (mock) or Pcnt in HEK293 cells. Cells were allowed to express the constructs for 48 hours before measuring Piezo2 mediated RA-MA currents. I did not observe any significant difference in Piezo2 RA-MA currents upon co-expression with mock or Pcnt (2-way ANOVA and post hoc Bonferroni test; Figure 16B). Note that Pcnt overexpression in HEK293 cells did not adversely affect cell health. The difference between DRG neurons and HEK293 cells could be explained by the compromised cell health in DRG neurons. There may also be a biological basis for the differences i.e. Pcnt may play different roles in post-mitotic DRG neurons compared to mitotic HEK293 cells (Buchman et al., 2010; Dammermann, 2002; Li et al., 2001; Zimmerman et al., 2004). Besides, the Piezo2-Pcnt interaction may require the presence of cell specific mediators that may be unavailable in HEK293 cells. 
A

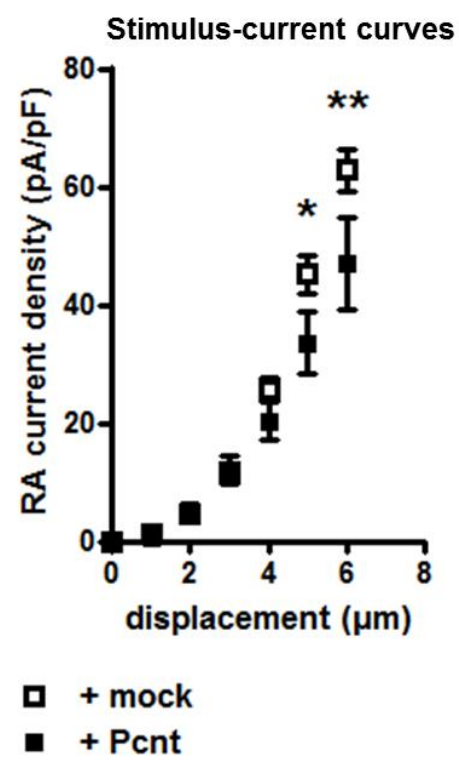

B

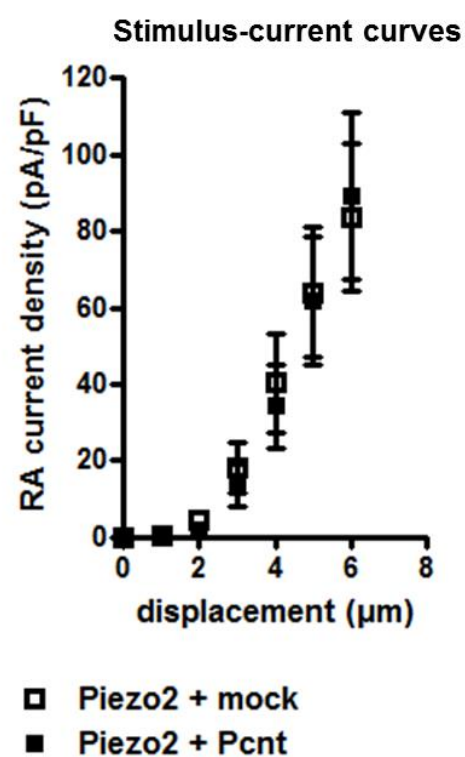

Figure 16: Pericentrin overexpression in DRG neurons and HEK293 cells

Stimulus-current curves of RA-MA currents upon overexpression of empty vector (mock) or Pericentrin plasmid in DRG neurons (A). Overexpression of Pcnt significantly decreases Piezo2 mediated RA-MA current magnitude ( $n=6-9$ cells per condition; 2-way ANOVA with post hoc Bonferroni test). It is important to note here that cellular health was severely compromised upon Pcnt overexpression in DRG neurons. Stimulus-current curves for overexpression of Piezo2 and Pcnt in HEK293 cells (B). Co-expression of Pcnt does not influence Piezo2 RA-MA currents compared to co-expression with mock plasmid ( $n=26-27$ neurons per condition; 2-way ANOVA with post hoc Bonferroni test). Reprinted (adapted) with permission from Narayanan et al. (2016). Copyright (2016) American Chemical Society. ${ }^{*}: p<0.05 ;{ }^{* *}: p<0.01 ;{ }^{* * *}: p<0.0001$ ${ }^{* * * *}: p<0.00001$; data are not significant unless otherwise stated.

\subsection{Mechanism of Piezo2-Pericentrin (Pcnt) interaction}

\section{Membrane expression of Piezo2 is increased upon Pericentrin knockdown}

In order to examine the cause of RA-MA potentiation, I checked for the Piezo2 expression upon loss of Pcnt. As shown in Figure 13B, mRNA of Piezo2 remains unchanged; hence membrane protein levels were tested. For this purpose, DRG neurons were cultured from the Piezo2 ${ }^{\text {GFP }}$ mice (Woo et al., 2014). The neurons were transfected with CTRL or Pcnt siRNA and maintained in culture for 72 hours prior to staining. To visualize the Piezo2 membrane levels, the cells were immunostained for GFP and WGA (Wheat germ agglutinin). WGA is a membrane dye that can be used to delineate cellular membranes, which facilitates the assessment of membrane Piezo2-GFP. Interestingly, I found a significant increase in the immunofluorescence of GFP on the membrane of the cells treated with Pcnt siRNA compared to CTRL (unpaired t-test; Figure 17A and B). 
Further, Poole and colleagues have shown that Piezo2 function depends critically on its substrate interactions (Poole et al., 2014). This prompted the question whether the Piezo2-GFP signal that I observed over the complete cellular membrane was also enhanced at the cell-substrate interface, upon Pcnt knockdown. For this, I analyzed the first five optical sections of the imaged cells (region closest to substrate) and found a similar increase at the cell-substrate interface (unpaired t-test; Figure 17C).

\section{A}

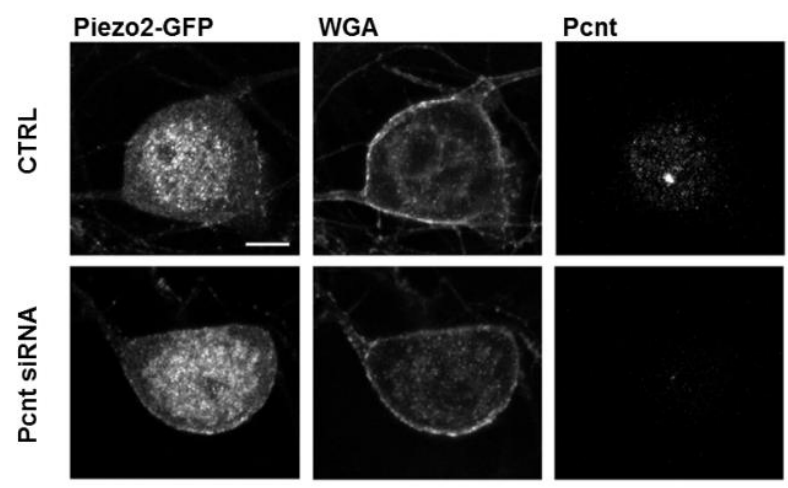

B

C
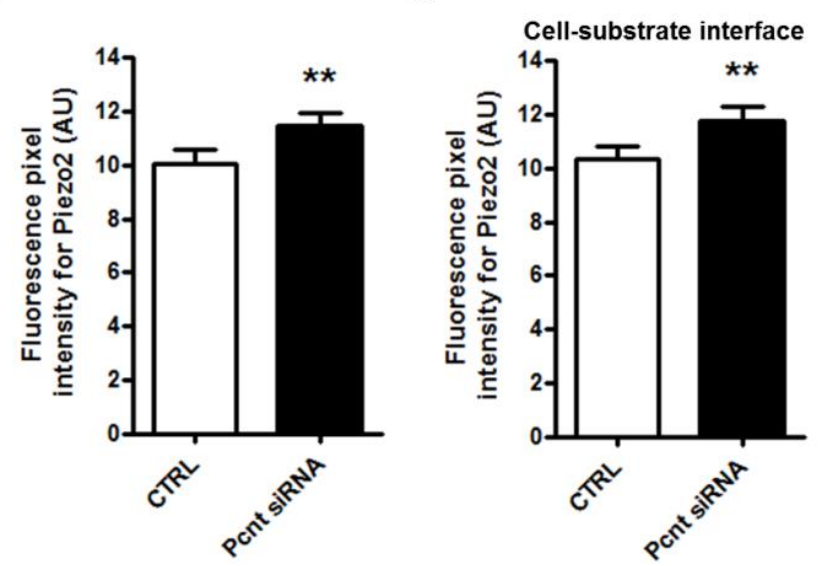

Figure 17: Exploring mechanisms of Piezo2-Pericentrin interaction

Representative images (A) and quantification (B, C) of membrane expression of Piezo2-GFP upon Pcnt knockdown in DRG neurons. Piezo2-GFP membrane expression is significantly increased upon Pcnt knockdown (Piezo2-GFP membrane intensity in CTRL: $10.07 \pm 0.50 \mathrm{AU}$; Pcnt siRNA: $11.46 \pm 0.52 \mathrm{AU}$; $n=50$ neurons per condition; $\mathrm{p}=0.0098$; unpaired $\mathrm{t}$-test). Membrane expression was also measured at cellsubstrate interface, where similar to whole cell membrane, Piezo2-GFP immunolabel is significantly increased in Pcnt knockdown DRG neurons (Piezo2-GFP membrane intensity in CTRL: $10.34 \pm 0.51 \mathrm{AU}$; Pcnt siRNA: $11.79 \pm 0.50 \mathrm{AU} ; n=50$ neurons per condition; $p=0.0089$; unpaired t-test). Scale bar $=10 \mu \mathrm{m}$. Stimulus-current curves for RA-MA currents (D) upon treatment of cells with anti-mitotic agent, Nocodazole $(35 \mu M)$. The treatment did not show any effect on RA-MA currents ( $n=12-16$ neurons per condition; 2-way ANOVA and post hoc Bonferroni test). Reprinted (adapted) with permission from Narayanan et al. (2016). Copyright (2016) American Chemical Society. ${ }^{*}: p<0.05 ;{ }^{* *}: p<0.01 ;{ }^{* * *}: p<0.0001 ;{ }^{* * *}: p<0.00001$; data are not significant unless otherwise stated. 


\section{Nocodazole treatment does not affect Piezo2 RA-MA currents}

From the earlier experiments, it has been established that Pcnt knockdown in DRG neurons increases Piezo2 mediated RA-MA currents which is supported by an increased Piezo2-GFP membrane expression. Considering that there is no change in the Piezo2 mRNA upon Pcnt knockdown, an increase in the trafficking of Piezo2 to the membrane is a likely scenario. From previous studies on Pcnt function, it is known that Pcnt is crucially involved in microtubule organization through its role in microtubule organization complex (MTOC) (Delaval and Doxsey, 2010). Based on this evidence, the Piezo2 phenotype upon Pcnt knockdown may be mediated by microtubule based transport. To test this, DRG neurons were treated with Nocodazole (Jurczyk et al., 2010) and Piezo2 mediated RA-MA currents were measured. Nocodazole is an anti-mitotic agent which disrupts microtubules by binding to $\beta$-tubulin. This in turn disrupts microtubule dynamics and has an effect on microtubule dependent trafficking. Surprisingly, microtubule disruption failed to influence Piezo2 mediated RA-MA currents (2-way ANOVA and post hoc Bonferroni test; Figure 17D).

These results point to a microtubule independent mode of Piezo2 trafficking and also indicate that the role of Pcnt in MTOC (microtubule organizing complex) may not be the major factor in the Piezo2-Pcnt interaction. 
56| RESULTS: PART 1

\subsection{Summary of part 1}

$>$ Pericentrin (Pcnt) was identified as a low probability interactor of Piezo2 through a immunoprecipitation and mass-spectrometry approach

$>$ Pcnt co-immunoprecipitates with Piezo2-GST (in HEK293 cells)

$>$ Loss of Pcnt in DRG neurons potentiates RA-MA (Piezo2 mediated) currents, without affecting the IA-MA or SA-MA currents

> Piezo2 membrane expression is increased in DRG neurons upon loss of Pcnt

> Piezo2 RA-MA currents appear to be independent of microtubule dynamics 


\section{RESULTS: Part 2}

\subsection{Verification of Piezo2-Mtmr2 interaction}

\section{Mtmr2 is expressed in the somatosensory system}

The myotubularin related protein 2 (Mtmr2) was identified as a potential interactor of Piezo2 in the mass spectrometry based interactomics screen. It was one of the high affinity interactors with a p-value of 0.00030 and a 9.96-fold enrichment compared to control (Narayanan et al., 2016). Mtmr2 has previously been described in the context of somatosensation and identified in mouse DRG neurons and Schwann cells (Previtali et al., 2003). Its mutation is linked to CMT4B1, a peripheral neuropathy (Bolino et al., 2004; Bolis et al., 2005; Previtali et al., 2007).

The first step in studying the Piezo2-Mtmr2 interaction was to characterize Mtmr2 expression in relation to Piezo2 in DRG neurons. For this purpose DRG tissue from Piezo2 ${ }^{\text {GFP }}$ mouse was used (Woo et al., 2014). The tissue was immunostained for GFP and Mtmr2, as described in the methods section (2.1.3). I found that $24.53 \pm 1.21 \% \mathrm{DRG}$ neurons were positive for GFP immunolabel indicating the presence of Piezo2 and 20.37 $\pm 2.01 \%$ DRG neurons were Mtmr2 immunolabel positive. Of the Piezo2 positive cells, $51.61 \pm 3.71 \%$ cells were found to be Mtmr2 positive (Figure 18). This indicates that Piezo2 and Mtmr2 are expressed in overlapping populations of DRG neurons. Of note, an RNA-seq analysis of mouse DRG showed a ubiquitous expression of Mtmr2 among all classified neuronal subtypes (Usoskin et al., 2015).

A

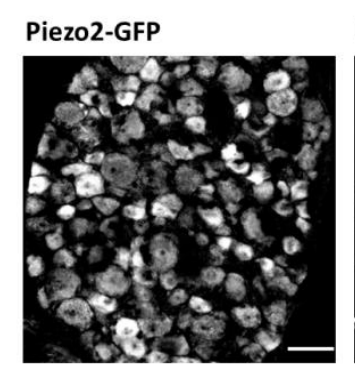

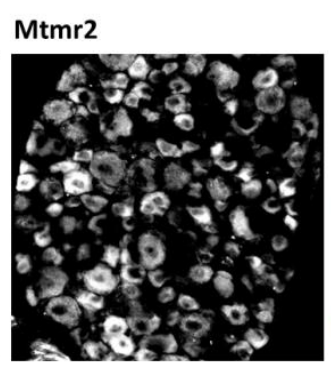

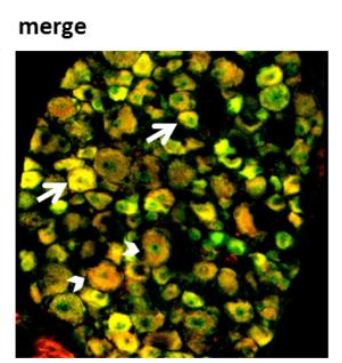

B

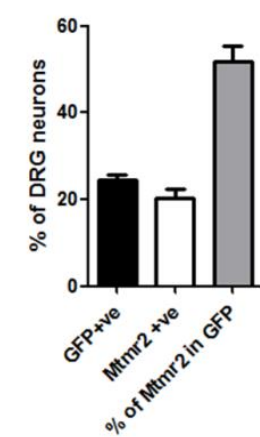

Figure 18: Mtmr2 expression in mouse DRG neurons

Representative images (A) and quantification of Piezo2-GFP and Mtmr2 in DRG neurons (B). DRG neurons from Piezo2 ${ }^{\text {GFP }}$ mice were immunolabelled for GFP $(24.53 \pm 1.21 \%)$ and Mtmr2 $(20.37 \pm 2.01 \%)$. A $51.61 \pm$ $3.71 \%$ overlap between Mtmr2 and Piezo2-GFP staining was observed ( $n>2000$ neurons from $N=3$ independent mice). Arrows indicate co-labelled cells while arrowheads mark Mtmr2 positive cells. Scale bar $=50 \mu \mathrm{m}$ 


\section{Mtmr2 and Piezo2 localize in close proximity in HEK293 cells}

Based on the finding that a subset of DRG neurons expresses both Piezo2 and Mtmr2, I asked if within these cells the two proteins were expressed in close proximity. Limitations of the anti-Mtmr2 antibody in primary cultures of DRG neurons prompted the use of heterologous HEK293 cells to study the interaction of Piezo2 and Mtmr2 within the cell.

Both proteins were co-expressed in HEK293 cells and proximity ligation assay (PLA) was carried out as described in the methods section (2.1.4).

Figure 19A shows representative PLA images of Piezo2 and Mtmr2 in HEK293 cells. I found a significantly higher PLA signal (puncta/area of cell) upon co-expression of Piezo2 with Mtmr2 compared to co-expression with mock plasmid (Mann Whitney test; Figure 19B). This difference was also clearly reflected in the fraction of positive area (Mann Whitney test; Figure 19C).

The results show that Piezo2 and Mtmr2 indeed localize in close proximity within HEK293 cells. Of note, the exact subcellular localization of Piezo2-Mtmr2 interaction was not probed, because the subcellular localization could be confounded by the overexpression system.

A

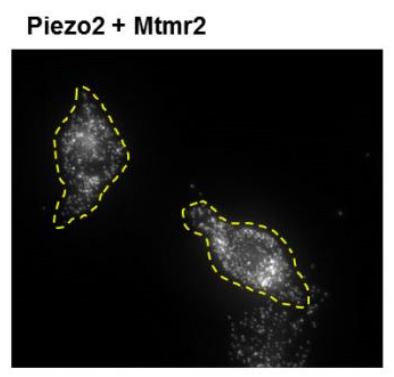

Piezo2 + mock

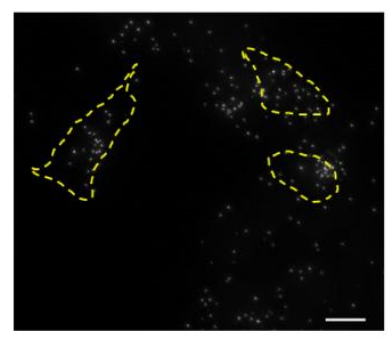

B

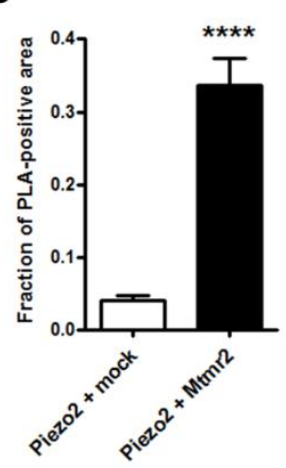

C

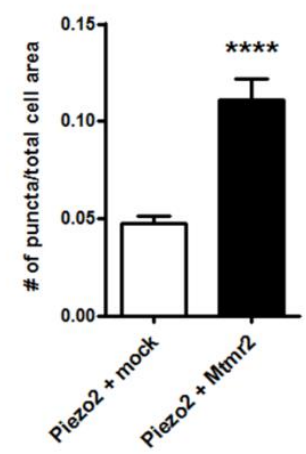

Figure 19: Piezo2 and Mtmr2 are expressed in close proximity in HEK293 cells

Representative images (A) and quantification of proximity ligation assay (PLA) for Piezo2 and Mtmr2 overexpressed in HEK293 cells (B). PLA signal is significantly higher when Piezo2 is co-expressed with Mtmr2 compared with mock plasmid. When quantified, the number of PLA puncta per cell area is significantly higher in Piezo2 + Mtmr2 compared to Piezo2 + mock (Piezo2 + mock: $0.05 \pm 0.004$ puncta/cell area; Piezo2 + Mtmr2: $0.11 \pm 0.01$ puncta/cell area; $n=54-60$ cells per condition; $p<0.0001$; Mann Whitney test). Fraction of positive area (C) was also significantly increased upon Piezo2 co-expression with Mtmr2 compared to mock (Piezo2 + mock: $0.04 \pm 0.007$; Piezo2 + Mtmr2: $0.34 \pm 0.04 ; n=54-60$ cells per condition; $p<0.0001$; Mann Whitney test). Dotted yellow lines have been used to demarcate cell boundaries. Scale bar $=10 \mu \mathrm{m} .{ }^{*}: p<0.05 ;{ }^{* *}: p<0.01 ;{ }^{* * *}: p<0.0001 ;{ }^{* * *}: p<0.00001$; data are not significant unless otherwise stated. 


\subsection{Functional characterization of Piezo2-Mtmr2 interaction in HEK293 cells}

\subsubsection{Mtmr2 co-expression suppresses Piezo2 RA-MA currents in HEK293 cells}

Piezo2 was overexpressed in HEK293 cells with either Mtmr2 or mock. MA currents were measured from these cells 48 hours after transfection.

Interestingly, Mtmr2 co-expression lead to a highly significant reduction in the Piezo2 mediated currents compared to mock (2-way ANOVA with Holm-Sidak's multiple comparison test; Figure 20A and B). Concomitantly, the activation threshold of the RAMA currents was significantly increased upon overexpression with Mtmr2 (Mann Whitney test; Figure 20C). (Of note, the number of cells for which activation threshold was measured is different from the cells in the stimulus-current curves because of the very low current magnitudes of Mtmr2 overexpressing HEK293 cells. Cells that did not cross the current magnitude even at $6 \mu \mathrm{m}$ stimulus were not included in the activation threshold analysis). Interestingly, the Stim $_{50}$ (stimulus required to elicit $50 \%$ of Imax) remained unchanged upon Mtmr2 co-expression (Mann Whitney test; Figure 20D). The inactivation time of the Piezo2 currents remained unchanged upon Mtmr2 co-expression with Piezo2 (Mann Whitney test; Figure 20E). The data indicate the Mtmr2 co-expression with Piezo2 suppresses Piezo2 mediated currents in HEK293 cells. Importantly, the defining property of RA-MA currents (fast inactivation $\tau$ ) remains unchanged upon Mtmr2 co-expression. 
A

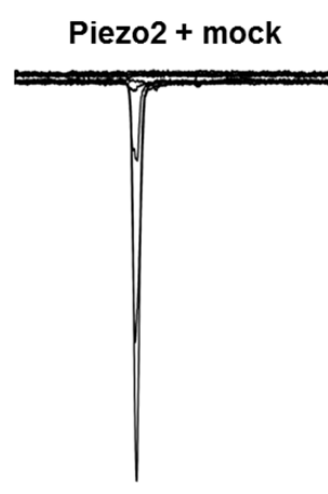

C

Activation threshold

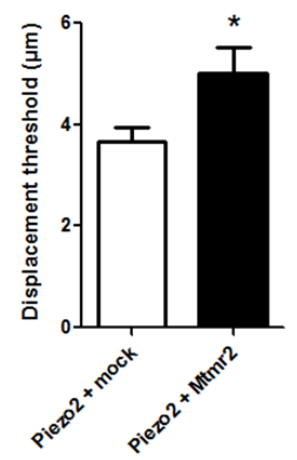

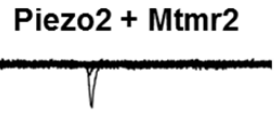

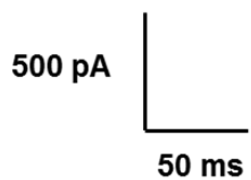

D

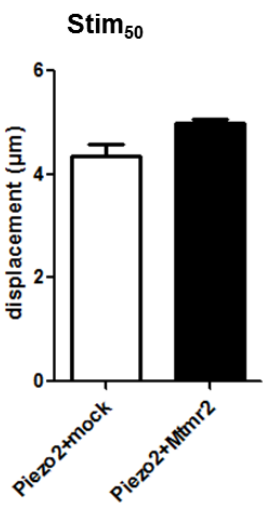

B

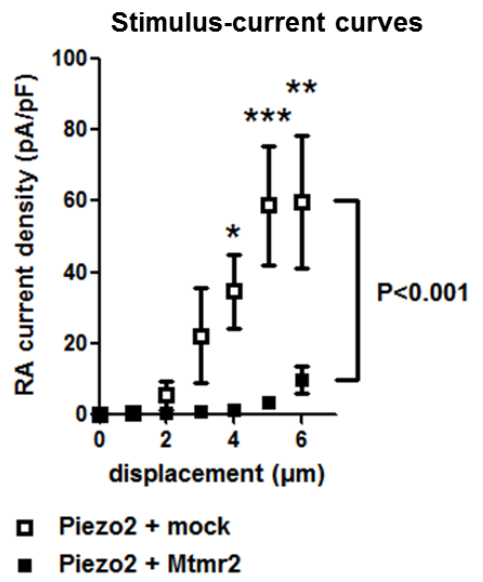

$\mathbf{E}$ Inactivation time constant

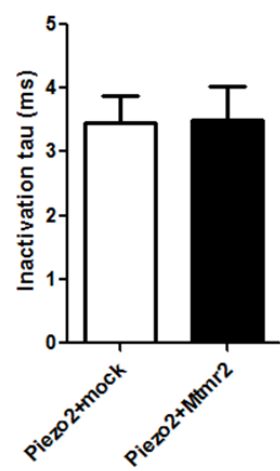

Figure 20: Mtmr2 co-expression with Piezo2 in HEK293 cells suppresses RA-MA currents

Representative traces of RA-MA currents in HEK293 cells overexpressing Piezo2 and mock or Piezo2 and Mtmr2 (A). Stimulus-current curves (B) show that Mtmr2 co-expression strongly suppresses Piezo2 mediated RA-MA currents in HEK293 cells (Piezo2 + mock: $n=11-17$ cells; Piezo2 + Mtmr2: $n=11-12$ cells; 2-way ANOVA reports a significant effect $(\mathrm{P}<0.001)$ Mtmr2 overexpression on RA-MA Piezo2 currents; Holm-Sidak's multiple comparison test was used to compare both conditions at individual stimulus magnitudes, results represented on the graph with '*'.). Activation threshold (C) of RA-MA currents upon Mtmr2 overexpression is significantly higher than mock (Piezo2 + mock: $3.67 \pm 0.29 \mu \mathrm{m}, \mathrm{n}=15$ cells; Piezo2 + Mtmr2: $5.00 \pm 0.52 \mu \mathrm{m}, \mathrm{n}=6$ cells; $\mathrm{p}=0.045$; Mann Whitney test). Stim ${ }_{50}$ of RA-MA currents (D) is unchanged upon Mtmr2 overexpression compared to mock (Piezo2 + mock: $4.34 \pm 0.24 \mu \mathrm{m}$; Piezo2 + Mtmr2: $4.98 \pm 0.09 \mu \mathrm{m} ; \mathrm{n}=11$ cells per condition; ns; Mann Whitney test). Inactivation time constant (T) of RA-MA currents $(E)$ was not significantly different between Piezo2 + mock and Piezo2 + Mtmr2 (Piezo2 + mock: $3.46 \pm 0.42 \mathrm{~ms}, \mathrm{n}=17$ cells; Piezo2 + Mtmr2: $3.48 \pm 0.56 \mathrm{~ms}, \mathrm{n}=12$ cells; $\mathrm{ns} ;$ Mann Whitney test). *: $p<0.05 ;{ }^{* *}: p<0.01 ;{ }^{* * *}: p<0.0001 ;{ }^{* * * *}: p<0.00001 ;$ data are not significant unless otherwise stated. 


\subsubsection{Mtmr2 co-expression does not affect Piezo1 mediated MA currents or voltage dependent Kv1.1 currents in HEK293 cells}

As shown in Figure 20B, Mtmr2 strongly suppresses Piezo2 mediated currents in HEK293 cells. In order to assess the specificity of this effect I measured Piezo1 mediated MA currents and Kv1.1 mediated voltage gated currents upon Mtmr2 overexpression. Interestingly, no significant difference was found either in Piezo1 currents or Kv1.1 currents upon co-expression of Mtmr2 compared to mock (2-way ANOVA with HolmSidak's multiple comparison test; Figure 21A and $\mathbf{B}$ respectively).

These results indicate that Mtmr2 co-expression does not in general suppress ion channel expression or function, and attests to the specificity of the Piezo2-Mtmr2 interaction. It also asserts that Mtmr2 overexpression does not affect the overall health of HEK293 cells.

A

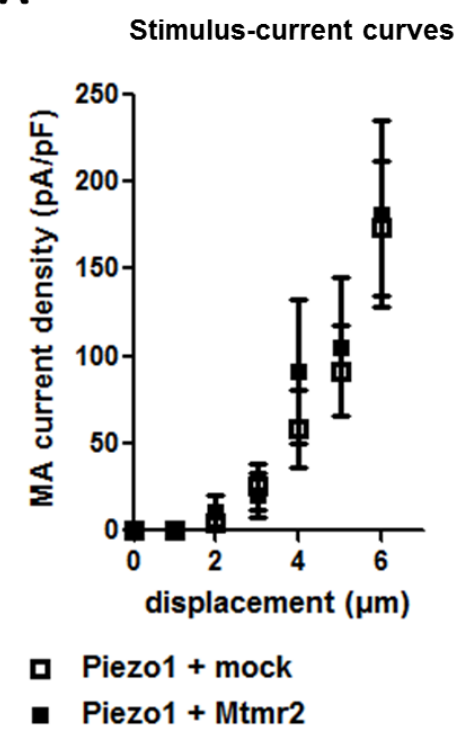

B

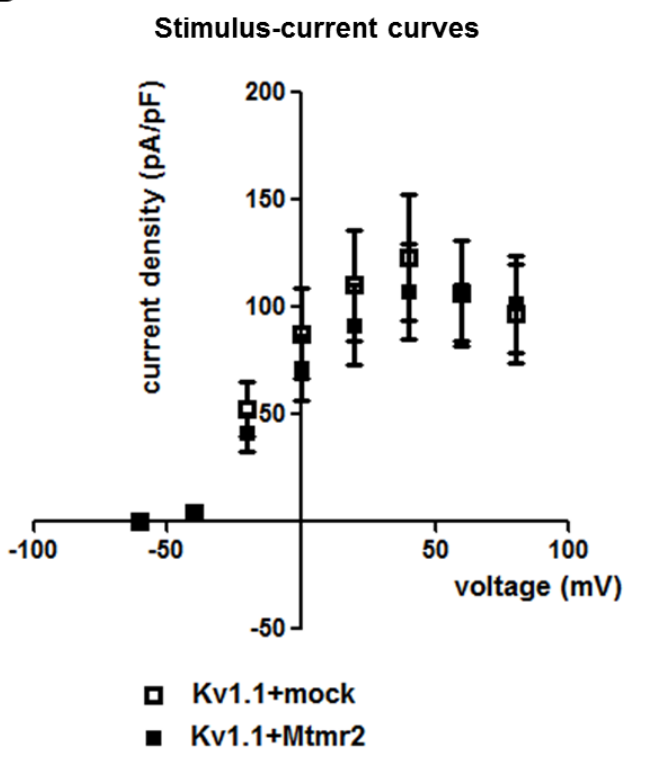

Figure 21: Mtmr2 co-expression does not affect Piezo1 or Kv1.1 currents in HEK293 cells

Stimulus-current curves (A) for Piezo1 currents in HEK293 cells co-expressed with mock or Mtmr2. Mtmr2 co-expression does not influence Piezo1 mediated currents (Piezo1 + mock: $n=19-25$ cells; Piezo1 + Mtmr2: $n=12-20$ cells; $n s ; 2$-way ANOVA with Holm-Sidak's multiple comparison test). Current-voltage curves (B) for Kv1.1 currents upon co-expression with mock or Mtmr2 plasmid. There is no significant effect of Mtmr2 coexpression on the Kv1.1 currents (Kv1.1 + mock: $n=23-24$ cells; Kv1.1 + Mtmr2: $n=25$ cells; ns; 2-way ANOVA with Holm-Sidak's multiple comparison test). ${ }^{*}: p<0.05 ;{ }^{* *}: p<0.01 ;{ }^{* * *}: p<0.0001 ;{ }^{* * *}: p<0.00001$; data are not significant unless otherwise stated. 


\subsection{Functional characterization of Piezo2-Mtmr2 interaction in DRG neurons \\ 4.3.1. Mtmr2 overexpression in DRG neurons decreases Piezo2 mediated current magnitude}

Based on the overexpression data in HEK293 cells it was important to study Piezo2Mtmr2 interaction in DRG neurons that express both proteins natively. For this purpose I first overexpressed Mtmr2 in DRG neurons to recapitulate the phenotype observed in HEK293 cells.

DRG neurons were cultured and transfected with the Mtmr2 construct and RA-MA currents were recorded 48 hours after transfection. Similar to HEK293 cells, Mtmr2 overexpression suppressed the native RA-MA currents in these neurons (Figure 22A). The stimulus-current curves show a significant decrease in the RA-MA current magnitude upon overexpression of Mtmr2 compared to mock (2-way ANOVA with Holm-Sidak's multiple comparison test; Figure 22B).

Surprisingly though, neither the activation threshold nor the Stim $_{50}$ of the RA-MA currents appears to have been significantly affected upon Mtmr2 overexpression (Mann Whitney test; Figure 22C and D). The difference in RA-MA regulation between HEK293 cells and DRG neurons could arise from either expression levels of the proteins, additional modulators that may be system exclusive or system specific differences in the Mtmr2Piezo2 interaction itself. To further characterize the Piezo2-Mtmr2 interaction in DRG neurons I used a siRNA based approach. 
A

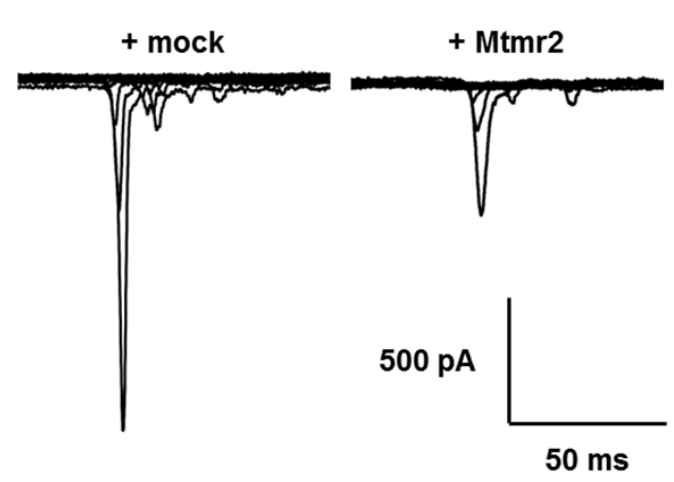

C

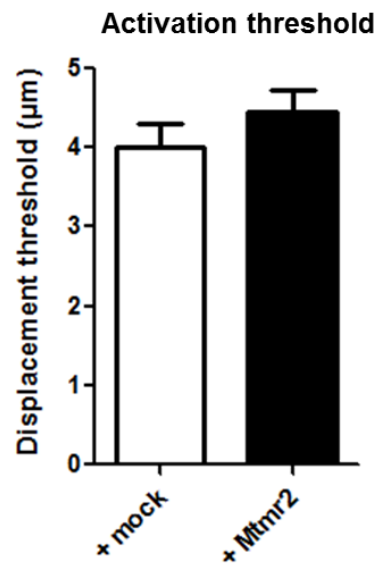

B

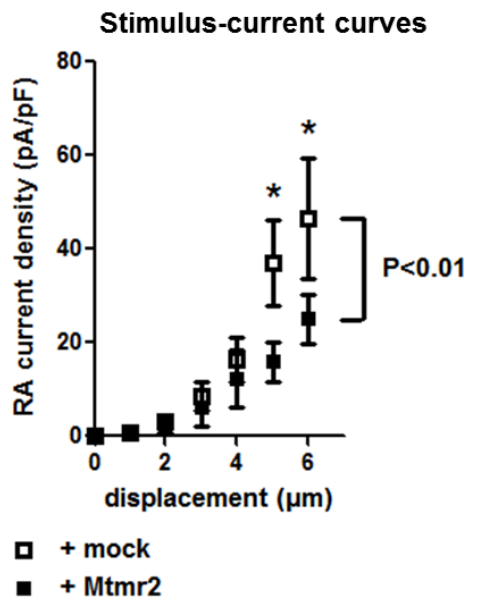

D

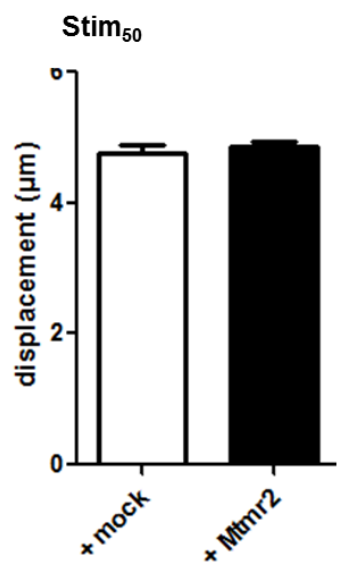

Figure 22: Mtmr2 overexpression in DRG neurons suppresses RA-MA currents

Representative RA-MA traces for overexpression of Mtmr2 or mock in DRG neurons (A). Stimulus-current curves (B) of RA-MA currents in DRG neurons upon Mtmr2 overexpression. Mtmr2 overexpression significantly decreases RA-MA currents in DRG neurons (+ mock: $n=22-28$ neurons; + Mtmr2: $n=27-30$ neurons; 2-way ANOVA reports a significant effect $(\mathrm{P}<0.01)$ of Mtmr2 overexpression on RA-MA Piezo2 currents; Holm-Sidak's multiple comparison test was used to compare both conditions at individual stimulus magnitudes, results are represented on the graph with '*'). Activation threshold of RA-MA currents (C) was unchanged upon overexpression of Mtmr2 (+ mock: $4.00 \pm 0.31 \mu \mathrm{m}, \mathrm{n}=23$ neurons; + Mtmr2: $4.46 \pm 0.26 \mu \mathrm{m}$ $\mathrm{n}=22$ neurons; ns; Mann Whitney test). Stim 50 of RA-MA currents (D) also remains unchanged upon Mtmr2 overexpression (+ mock: $4.74 \pm 0.14 \mu \mathrm{m}, \mathrm{n}=23$ neurons; + Mtmr2: $4.86 \pm 0.08 \mu \mathrm{m}, \mathrm{n}=28$ neurons; $\mathrm{ns}$; Mann Whitney test). ${ }^{*}: p<0.05 ;{ }^{* *}: p<0.01 ;{ }^{* * *}: p<0.0001 ;{ }^{* * *}: p<0.00001$; data are not significant unless otherwise stated. 


\subsubsection{Mtmr2 siRNA in DRG neurons effectively reduces Mtmr2 expression without affecting Piezo2}

\section{Mtmr2 mRNA is decreased upon Mtmr2 knockdown in DRG neurons}

Primary cultures of DRG neurons were transfected with Mtmr2 siRNA as described in the methods section (2.2.1). All analysis was done 72 hours after transfection. In order to assess the efficiency of the siRNA, quantitative PCR was performed as described in the methods section (2.2.2). Amount of Mtmr2 mRNA was quantified with reference to housekeeping genes GAPDH and actin. Mtmr2 siRNA significantly reduced the Mtmr2 mRNA compared to CTRL relative to both housekeeping genes (One sample t-test; Figure 23A).

Further, Piezo2 mRNA was also analyzed upon Mtmr2 knockdown and no significant difference was observed compared to CTRL (One sample t-test; Figure 23B). The above data show that the Mtmr2 siRNA is highly effective in reducing Mtmr2 mRNA in DRG neuron cultures, while Piezo2 mRNA remains unaffected.

A

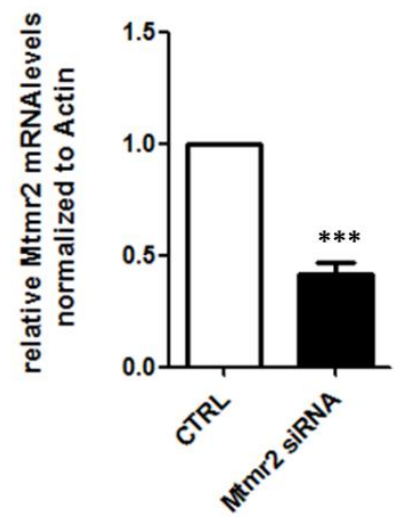

C

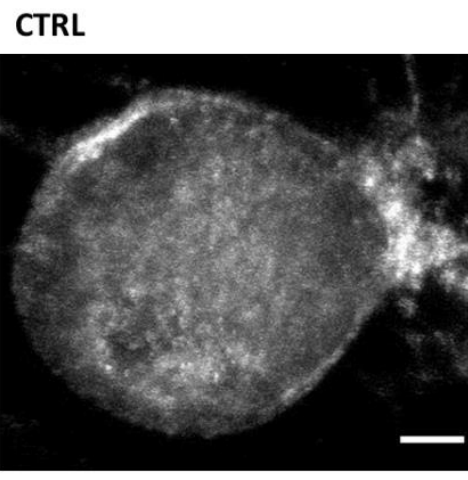

B

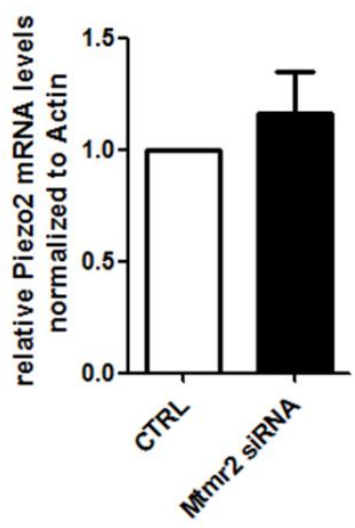

D
Mtmr2 siRNA

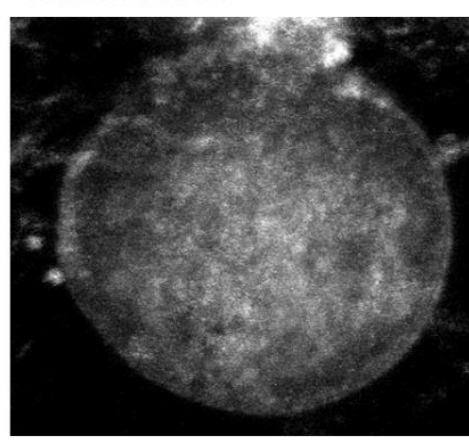

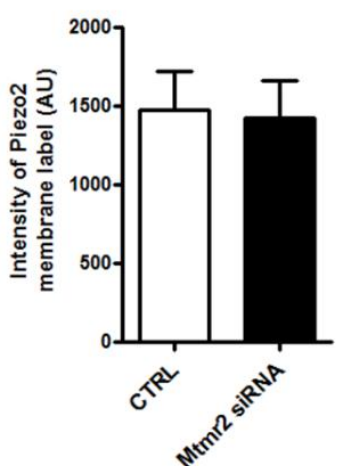


Figure 23: Knockdown of Mtmr2 in DRG neurons

Mtmr2 siRNA mediated knockdown in DRG neurons (A) significantly decreases Mtmr2 (Mtmr2 mRNA upon Mtmr2 siRNA, actin as reference: $0.42 \pm 0.05 ; p<0.0001$; One sample t-test; $N=8$ DRG cultures). No change in Piezo2 mRNA (B) was observed (Piezo2 mRNA upon Mtmr2 siRNA, actin as reference: $1.17 \pm 0.18$; ns; One sample t-test; N=8 DRG cultures). Representative images (C) and quantification (D) of Piezo2 membrane intensity upon Mtmr2 knockdown in DRG neurons. Membrane immunofluorescence of Piezo2GFP remained unchanged upon Mtmr2 knockdown (CTRL: $1470 \pm 246.7$ AU; Mtmr2 siRNA: $1424 \pm 237.5$ $\mathrm{AU}$; ns; Mann Whitney test; $\mathrm{n}=22$ neurons per condition). Scale bar $=5 \mu \mathrm{m}$. ${ }^{*}: \mathrm{p}<0.05 ;{ }^{* *}: \mathrm{p}<0.01 ;{ }^{* * *}$ : $p<0.0001 ;{ }^{* * * *}: p<0.00001$; data are not significant unless otherwise stated.

\section{Piezo2 membrane expression is unchanged upon Mtmr2 knockdown}

To study the effect of Mtmr2 knockdown on Piezo2 in DRG neurons, membrane expression of Piezo2 was measured using immunolabelling. DRG neurons from Piezo2 ${ }^{\text {GFP }}$ mice (Woo et al., 2014) were transfected with Mtmr2 siRNA and immunolabelled against GFP, 72 hours post transfection. Analysis of membrane intensity was done as described in the methods section (2.2.4). I found no significant difference in the membrane intensity of Piezo2-GFP staining upon transfection with Mtmr2 siRNA compared to CTRL (Mann Whitney test; Figure 23C and D). In conclusion, Mtmr2 knockdown changes neither Piezo2 mRNA levels nor the membrane expression of Piezo2.

\subsubsection{Mtmr2 knockdown potentiates Piezo2 mediated MA currents}

As described earlier Mtmr2 expression was suppressed in DRG neurons using siRNA. RA-MA currents were measured from the cells 72 hours after transfection. Remarkably, the RA-MA current magnitude was increased upon knockdown of Mtmr2 compared to CTRL (2-way ANOVA with Holm-Sidak's multiple comparison test; Figure 24B). Further analysis revealed that the activation threshold of the RA-MA currents was significantly decreased upon Mtmr2 knockdown (Mann Whitney test; Figure 24C). I also analyzed the Stim $_{50}$ of the RA-MA currents and found no significant difference between CTRL and Mtmr2 siRNA conditions (Mann Whitney test; Figure 24D). It is important to note here that the MA current data cannot be compared between the different experiments namely overexpression of Mtmr2 and knockdown of Mtmr2, because of the differences in the transfection conditions and recording days in vitro (DIV). Hence each set of data was performed with its own controls. 
The results show that Mtmr2 is capable of bi-directional regulation of Piezo2 RA-MA currents in DRG neurons

A

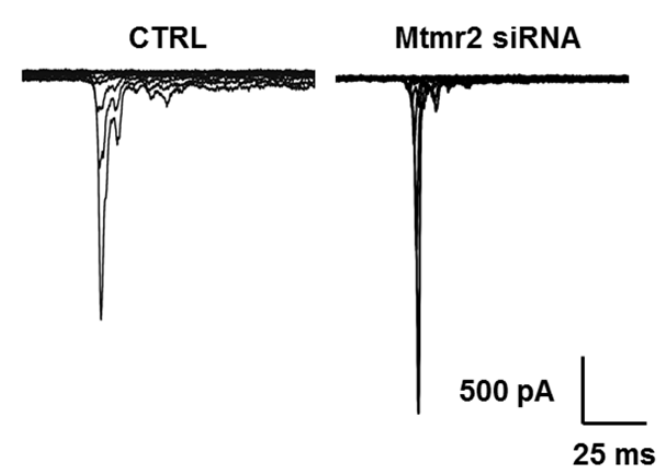

B

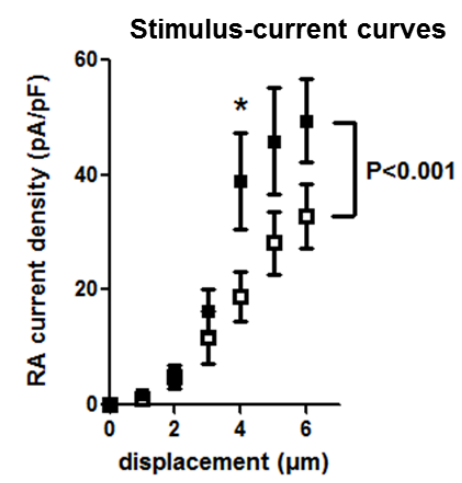

a CTRL

- Mtmr2 siRNA

D

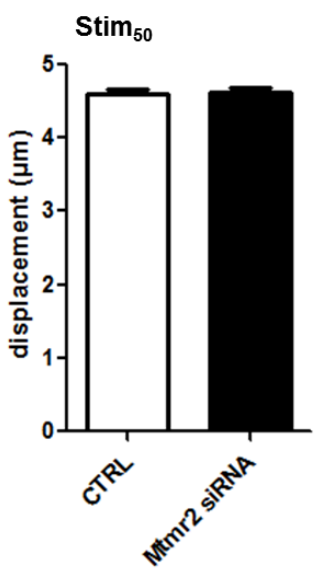

\section{Figure 24: Mtmr2 knockdown in DRG neurons potentiates RA-MA currents}

Representative traces of RA-MA currents in CTRL and Mtmr2 siRNA treated DRG neurons (A). Stimuluscurrent curves (B) show that Mtmr2 knockdown leads to significant increase in the Piezo2 mediated RA-MA currents (CTRL: $n=59-68$ neurons; Mtmr2 siRNA: $n=63-78$ neurons; 2-way ANOVA reports a significant effect $(P<0.001)$ of the loss of Mtmr2 on RA-MA Piezo2 currents; Holm-Sidak's multiple comparison test was used to compare both conditions at individual stimulus magnitudes, results are represented on the graph with (*)). Activation threshold (C) of Mtmr2 knockdown neurons is significantly lower than CTRL (CTRL: 3.95 $\pm 0.17 \mu \mathrm{m}, \mathrm{n}=65$ neurons; Mtmr2 siRNA: $3.44 \pm 0.17, \mathrm{n}=75$ neurons; $\mathrm{p}=0.033$; Mann Whitney test). Stim 50 of RA-MA currents (D) remains unchanged upon Pcnt knockdown (CTRL: $4.61 \pm 0.06 \mu \mathrm{m}, \mathrm{n}=58$ neurons; Mtmr2 siRNA: $4.62 \pm 0.06, n=63$ neurons; ns; Mann Whitney test). Of note, current traces and magnitude cannot be compared to data from other experiments because of differences in days in vitro (DIV) of recording and different transfection treatments. Hence all independent experiments were performed with respective controls. ${ }^{*}: p<0.05 ;{ }^{* *}: p<0.01 ;{ }^{* * *}: p<0.0001 ;{ }^{* \star *}: p<0.00001$; data are not significant unless otherwise stated. 


\subsubsection{Mtmr2 knockdown does not affect other MA currents but affects population distribution of MA currents}

As mentioned in the section 1.2.2, DRG neurons display three types of MA currents based in their inactivation time constant $(\tau)$.

Two of these MA currents, intermediate and slowly adapting currents (IA and SA) are not directly Piezo2 mediated (Coste et al., 2010; Dubin et al., 2012; Ranade et al., 2014) and can therefore be used to study the specificity of Piezo2-Mtmr2 interaction.

As shown in Figure 25A and $B$ neither the IA-MA nor the SA-MA currents were significantly changed upon Mtmr2 knockdown (2-way ANOVA with Holm-Sidak's multiple comparison test; Figure 25A and $B$ respectively).

This data provides additional proof for the specificity of the Piezo2-Mtmr2 interaction (in HEK293 cells it was seen that Mtmr2 does not interact with Piezo1 and Kv1.1; Figure 21).

Besides single cell properties, I also looked at the population of the MA currents in the DRG cultures treated with CTRL or Mtmr2 siRNA. Surprisingly, I found that there was a significant redistribution of the MA currents upon Mtmr2 knockdown. There was an increase in the proportion of RA-MA exhibiting cells with a concomitant decrease in the IA-MA exhibiting population (Chi-Square test; Figure 25C). This was a surprising observation considering there is no indication for the involvement of Piezo2 in the IA-MA. Surprisingly, the number of Piezo2 positive DRG neurons remains unchanged upon Mtmr2 knockdown (\% of Piezo2-GFP positive cells in CTRL: $29.59 \pm 4.97 \%$; Mtmr2 siRNA: $33.59 \pm 2.45 \%$; ns; Mann Whitney test; $N=3$ DRG cultures; data not shown) and no change in the overall Piezo2 mRNA was found (Figure 23B). Considering that the increased population of RA-MA currents is not contributed by increased number of Piezo2 positive cells, it is possible that Mtmr2 interacts with other ion channels (including the one generating IA-MA currents) and modulates their properties. Further understanding of this data awaits the characterization of the ion channels involved in generating IA-MA currents (Viatchenko-Karpinski and Gu, 2016). 
A

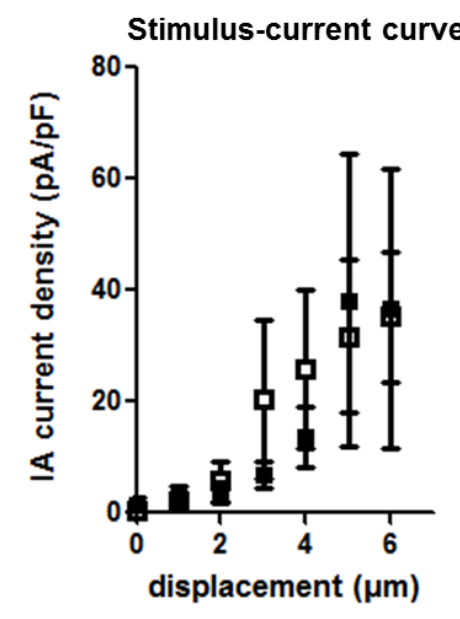

- CTRL

- Mtmr2 siRNA
B

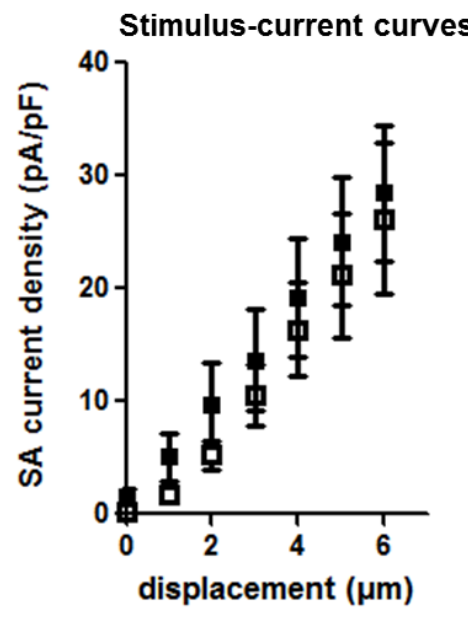

a CTRL

- Mtmr2 siRNA

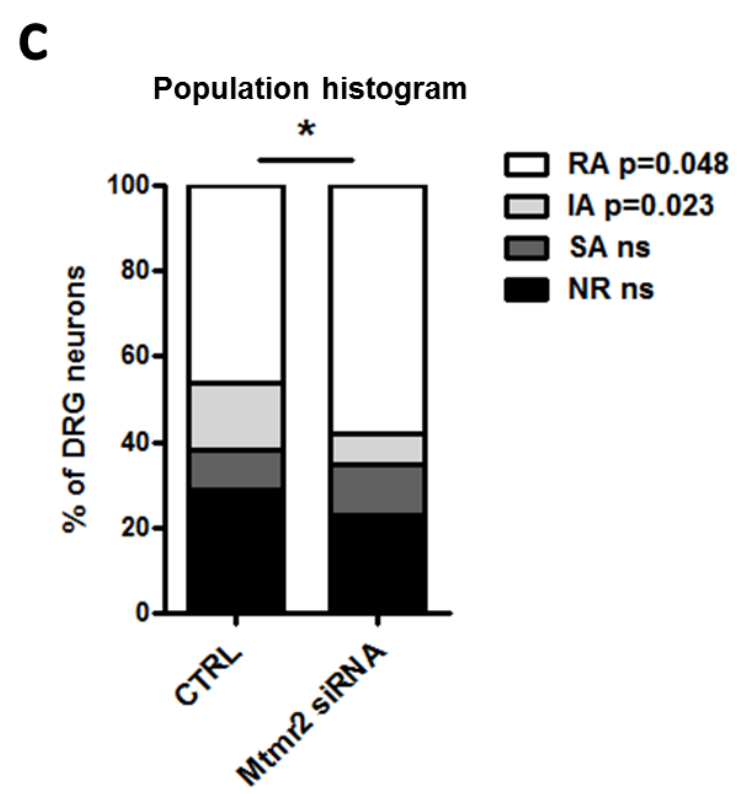

Figure 25: Mtmr2 knockdown does not modulate IA-MA and SA-MA currents

Stimulus-current curves of IA (A) and SA (B)-MA currents under CTRL or Mtmr2 siRNA conditions show no significant differences (IA-MA: CTRL: $n=24$ neurons; Mtmr2: $n=9-10$ neurons; ns. SA-MA: $n=16-17$ neurons per condition; ns; 2-way ANOVA with Holm-Sidak's multiple comparison test). Stacked histogram showing distribution of RA-MA, IA-MA, SA-MA and NR (non-responder) neurons in DRG cultures (C) treated with CTRL or Mtmr2 siRNA. RA-MA currents are significantly increased while the IA-MA currents are concomitantly decreased upon Mtmr2 knockdown (RA:IA:SA:NR; CTRL: 46:16:9:29; Mtmr2 siRNA: 58:7:12:23; $p=0.0439$; Chi-Square test; $>130$ neurons were analyzed per condition). Chi-square test for RAMA/total: $p=0.048$ and IA-MA/total: $p=0.023$. Numbers have been rounded off to reflect whole numbers. *: $p<0.05 ;{ }^{* *}: p<0.01 ;{ }^{* * *}: p<0.0001 ;{ }^{* * * *}: p<0.00001$; data are not significant unless otherwise stated. 


\subsection{Probing mechanism of Piezo2-Mtmr2 interaction}

In order to elucidate the mechanism of the Piezo2-Mtmr2 interaction, I focused on the cellular role of Mtmr2. As shown in Figure 8B, Mtmr2 is a phosphatase that catalysis the removal of the 3-phosphate group from $\mathrm{PI}(3) \mathrm{P}$ and $\mathrm{PI}(3,5) \mathrm{P}_{2}$ (Berger et al., 2002). PIPs have been known to regulate ion channels (Gamper and Shapiro, 2007) and recent evidence also suggests that Piezo2 can be modulated by phosphatidylinositols (Borbiro et al., 2015; Jia et al., 2016).

In light of this information, certain fundamental questions were asked. A) Is the catalytic role of Mtmr2 relevant to Mtmr2-Piezo2 interaction? B) Are the PIPs involved in the Mtmr2-Piezo2 interaction? and C) Can PIPs directly modulate Piezo2?

\subsubsection{Catalytically inactive Mtmr2 C417S does not influence RA-MA currents}

To know if the catalytic activity of Mtmr2 has a role to play in the Piezo2-Mtmr2 interaction, I generated an Mtmr2 mutant construct containing the C417S mutation (cysteine at position 417 modified to serine). This mutation renders the phosphatase catalytically inactive (Berger et al., 2002). If the catalysis of $\mathrm{PI}(3,5) \mathrm{P}_{2}$ and $\mathrm{PI}(3) \mathrm{P}$ would be essential to the interaction of Piezo2-Mtmr2, the catalytically inactive mutant would fail to recapitulate the Piezo2 RA-MA suppression caused by the overexpression with wild type Mtmr2, in HEK293 cells (Figure 20B). Extraordinarily, this was indeed found to be the case. The Mtmr2C417S construct fails to suppress Piezo2 mediated currents in HEK293 cells. In fact, the overexpression with Mtmr2C417S caused a mild but significant increase in the RA-MA currents. Stimulus-current curves (2-way ANOVA with Holm-Sidak's multiple comparison test; Figure 26A) show the small increase of RA-MA currents upon co-expression with Mtmr2C417S. Activation threshold (Mann Whitney test; Figure 26B) was unchanged upon overexpression of Piezo2 with the Mtmr2C417S construct. This result strongly suggests that Mtmr2 catalytic activity is essential for it to modulate Piezo2 currents and hints to the possibility of $\mathrm{PI}(3,5) \mathrm{P}_{2}$ and $\mathrm{PI}(3) \mathrm{P}$ being involved in the interaction. In order to check if the proximity of Piezo2 and Mtmr2 is affected by the C417S mutation, I performed proximity ligation assay. Interestingly, Mtmr2C417S despite its loss of enzymatic activity still localizes in close proximity to Piezo2 in HEK293 cells. Figure 26C shows representative PLA images of Piezo2 co-expressed with Mtmr2, Mtmr2C417S or mock in HEK293 cells. A significantly higher PLA signal was observed (puncta/area of cell) upon co-expression of Piezo2 with Mtmr2C417 compared to coexpression with Mock plasmid. 
There was no difference between the PLA signal upon co-expression of Piezo2 with Mtmr2 or Mtmr2C417S (Kruskal Wallis test with Dunn's Multiple Comparison Test; Figure 26D). Similar results were obtained when comparing fraction of positive area (Kruskal Wallis test with Dunn's Multiple Comparison Test; Figure 26E). This data indicates that enzymatically inactive Mtmr2 (Mtmr2C417S) remains capable of associating with Piezo2 but loses the ability to modulate Piezo2 RA-MA currents.

A

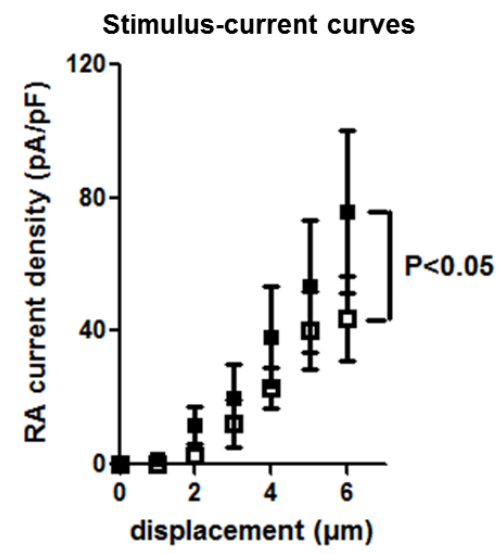

- Piezo2 + mock

- Piezo2 + Mtmr2C417S

C

Piezo2+Mtmr2C417S
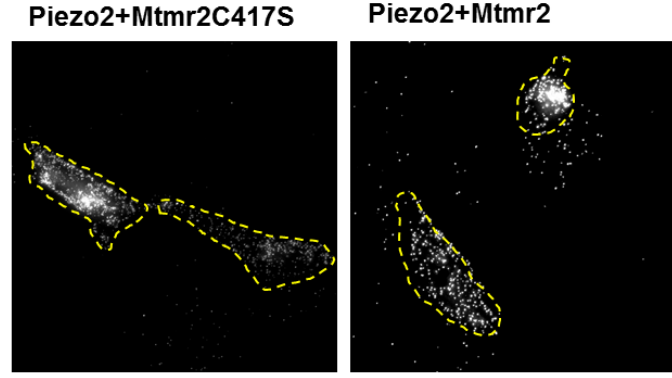

D

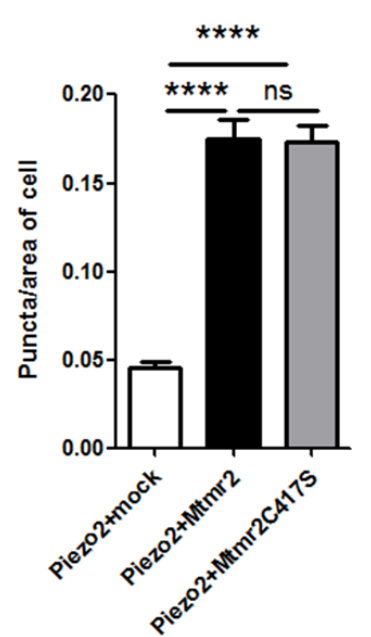

B

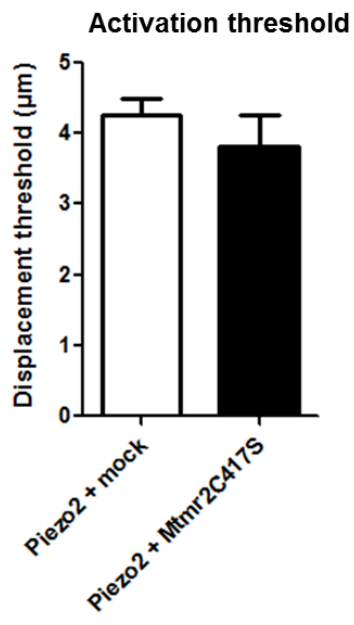

Piezo2+Mock

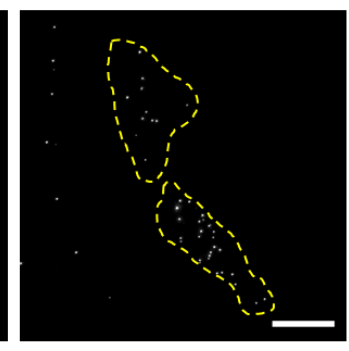

E

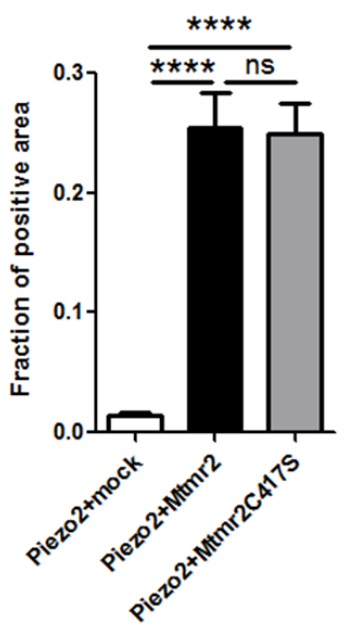




\section{Figure 26: Mtmr2C417S does not affect Piezo2 currents in HEK293 cells}

Stimulus-current curves for Piezo2 RA-MA currents in HEK293 cells upon co-expression with mock or Mtmr2C417S construct (A). Mtmr2C417S co-expression causes a mild but significant potentiation of Piezo2 RA-MA currents (Piezo2+mock: $n=24-33$ cells; Piezo2+Mtmr2C417S: $n=14-18$ cells; $P<0.05$; 2-way ANOVA. Holm-Sidak's multiple comparison test shows no significant differences at individual stimulus magnitudes). Activation properties (B) of RA-MA currents remain unchanged upon overexpression of Piezo2 with Mtmr2C417S compared to overexpression with mock (Piezo2+mock: $4.26 \pm 0.24 \mu \mathrm{m}, \mathrm{n}=27$ cells; Piezo2+Mtmr2C417S: $3.81 \pm 0.44 \mu \mathrm{m}, \mathrm{n}=16$ cells; $\mathrm{ns}$; Mann Whitney test). Representative images of proximity ligation assay for co-expression of Piezo2 with mock, Mtmr2 and Mtmr2C417S (C). PLA signal is significantly higher when Piezo2 is co-expressed with Mtmr2 or with Mtmr2C417S compared with mock plasmid. Both puncta/area of cell (D) and fraction of positive area (E) show that Mtmr2 and Mtmr2 C417S localize in close proximity to Piezo2 in HEK293 cells.

(Puncta/cell: Piezo2 + mock: $0.05 \pm 0.003$ puncta/cell, $n=75$ cells; Piezo2 + Mtmr2: $0.18 \pm 0.01$ puncta/cell, $\mathrm{n}=70$ cells; Piezo2 + Mtmr2C417S: $0.17 \pm 0.09$ puncta/cell, $\mathrm{n}=70$ cells; Piezo2 + mock versus Piezo2 + Mtmr2: $p<0.0001$; Piezo2 + mock versus Piezo2 + Mtmr2C417S: $p<0.0001 ;$ Piezo2 + Mtmr2 versus Piezo2 + Mtmr2C417S: ns; Kruskal Wallis test with Dunn's Multiple Comparison Test).

(Fraction of positive area: Piezo2 + mock: $0.01 \pm 0.003, n=75$ cells; Piezo2 + Mtmr2: $0.25 \pm 0.03, n=70$ cells; Piezo2 + Mtmr2C417S: $0.25 \pm 0.03, n=70$ cells; Piezo2 + mock versus Piezo2 + Mtmr2: $p<0.0001 ;$ Piezo2 + mock versus Piezo2 + Mtmr2C417S: $p<0.0001$; Piezo2 + Mtmr2 versus Piezo2 + Mtmr2C417S: ns; Kruskal Wallis test with Dunn's Multiple Comparison Test). Yellow dotted lines have been used to demarcate cell boundaries. Scale bar $=20 \mu \mathrm{m} .{ }^{*}: \mathrm{p}<0.05 ;{ }^{* *}: p<0.01 ;{ }^{* * *}: p<0.0001 ;{ }^{* * *}: p<0.00001$; data are not significant unless otherwise stated.

\subsubsection{Apilimod (and to a lesser extent Wortmannin) application can 'reverse' Mtmr2 knockdown phenotype}

Apilimod decreases RA-MA currents which were increased upon Mtmr2 knockdown $\underline{\text { neurons }}$

To answer if the PIPs are involved in the Piezo2-Mtmr2 interaction I used pharmacological manipulation of the PIP synthesis and turnover pathway. As shown in Figure 27A, Apilimod is a specific inhibitor of PIKfyve, the enzyme that generates $\mathrm{PI}(3,5) \mathrm{P}_{2}$ (Cai et al., 2013; Messenger et al., 2015). From the figure it can also be seen that decreasing Mtmr2 would cause an increase in the levels of $\mathrm{PI}(3,5) \mathrm{P}_{2}$. Therefore if $\mathrm{PI}(3,5) \mathrm{P}_{2}$ would be a relevant component of the Piezo2-Mtmr2 interaction, Apilimod application would be expected to 'rescue/reverse' the increased RA-MA currents caused by knockdown of Mtmr2. With this in mind, Mtmr2 knockdown DRG neurons were treated with Apilimod as described in the methods section (2.2.6). Figure 27B shows the stimulus-current curves for RA-MA currents in DRG neurons. Apilimod application significantly reduces the magnitude of RA-MA currents in Mtmr2 knockdown neurons (2way ANOVA with Holm-Sidak's multiple comparison test; Figure 27B). Concomitantly, 
the activation threshold of the RA-MA currents was increased significantly upon Apilimod treatment (Mann Whitney test; Figure 27D). This result points to a role for $\mathrm{PI}(3,5) \mathrm{P}_{2}$ in the Piezo2-Mtmr2 interaction.

Interestingly, Apilimod application on wild type DRG neurons did not have an impact on the RA-MA current magnitude (2-way ANOVA with Holm-Sidak's multiple comparison test; Figure 27E) or on activation threshold (Mann Whitney test; Figure 27F). This result may be explained by the low cellular expression of $\mathrm{PI}(3,5) \mathrm{P}_{2}$ under physiological conditions (Jin et al., 2016; McCartney et al., 2014a). Therefore an Apilimod mediated decrease of the already low $\mathrm{PI}(3,5) \mathrm{P}_{2}$ in wild type DRG neurons may not produce a cellular effect.

Wortmannin shows mild effect on RA-MA currents in Mtmr2 knockdown neurons

In cellular systems, Mtmr2 has two substrates $\mathrm{PI}(3,5) \mathrm{P}_{2}$ and $\mathrm{PI}(3) \mathrm{P}$. In the above section I looked at the role of the former because it was shown to be the preferential substrate (Berger et al., 2002). Following those results, I asked if PI(3)P may also be involved in the Piezo2-Mtmr2 interaction. Towards this goal, Wortmannin an inhibitor of the class III PI 3-kinase was used (Messenger et al., 2015). Mtmr2 knockdown DRG neuron cultures were treated with Wortmannin and RA-MA currents recorded. This treatment showed a mild but significant effect on RA-MA currents, as seen from the 2-way ANOVA. Though at the individual stimulus magnitudes, the two conditions were not significantly different. (2way ANOVA with Holm-Sidak's multiple comparison test; Figure 27C).

The activation threshold of cells treated with Wortmannin did not significantly differ from the cells treated with DMSO (Mann Whitney test; Figure 27D). These results suggest that $\mathrm{PI}(3) \mathrm{P}$ may play a small role in the Piezo2-Mtmr2 interaction. The relevance of this role and how it co-relates with $\mathrm{PI}(3,5) \mathrm{P}_{2}$ in Piezo2 regulation remains to be assessed. 
Figure 27: Mtmr2 knockdown can be rescued by Apilimod application

Schematic illustration of the $\mathrm{PI}(3) \mathrm{P}$ and $\mathrm{PI}(3,5) \mathrm{P}_{2}$ synthesis and turnover pathway, indicating the action of Apilimod and Wortmannin, which are blockers of PIKfyve and class III PI3-Kinase respectively (A). Stimuluscurrent curves of Piezo2 RA-MA currents in Mtmr2 knockdown neurons upon application of Apilimod (B). Apilimod treatment significantly reduces RA-MA currents (Mtmr2 siRNA+DMSO: $\mathrm{n}=21-27$ neurons; Mtmr2 siRNA+Apilimod: $n=24-30$ neurons; 2-way ANOVA reports that Apilimod treatment has a significant effect $(\mathrm{P}<0.001)$ on RA-MA Piezo2 currents; Holm-Sidak's multiple comparison test was used to compare both conditions at individual stimulus magnitudes, results are represented on the graph with '*'). Stimulus-current curves of RA-MA current in Mtmr2 knockdown DRG neurons upon application of Wortmannin (C). Wortmannin application has a significant effect on RA-MA currents (Mtmr2 siRNA+DMSO: $n=21-27$ neurons; Mtmr2 siRNA+Wortmannin: $n=27-29$ neurons; 2-way ANOVA ( $<<0.01$ ); Holm-Sidak's multiple comparison test showed no significant difference between individual current densities at different stimulus intensities). Activation threshold of RA-MA currents (D) is increased upon Apilimod treatment (Mtmr2 siRNA+DMSO: $3.52 \pm 0.24 \mu \mathrm{m}, \mathrm{n}=26$ neurons; Mtmr2 siRNA+Apilimod: $4.36 \pm 0.28 \mu \mathrm{m}, \mathrm{n}=27$ neurons; $\mathrm{p}=0.0179$; Mann Whitney test), while Wortmannin treatment did not change the activation threshold of RA-MA currents compared to DMSO application (Mtmr2 siRNA+DMSO: $3.52 \pm 0.24 \mu \mathrm{m}$; Mtmr2 siRNA+Wortmannin: $4.07 \pm$ $0.24 \mu \mathrm{m} ; \mathrm{ns} ; \mathrm{n}=27$ neurons per condition; Mann Whitney test). Stimulus-current curves of native RA-MA currents upon application of Apilimod (E). Apilimod application in wild type DRG neurons does not affect the Piezo2 RA-MA currents (+DMSO: $n=14-16$ neurons; +Apilimod: $n=8-13$ neurons; 2-way ANOVA with HolmSidak's multiple comparison test). Activation properties of RA-MA currents $(\mathbf{F})$ were also unchanged (+ DMSO: $3.88 \pm 0.44 \mu \mathrm{m}, \mathrm{n}=16$ neurons; + Apilimod: $3.31 \pm 0.52 \mu \mathrm{m}, \mathrm{n}=13$ neurons; $\mathrm{ns}$; Mann Whitney test). ${ }^{*}: p<0.05 ;{ }^{* *}: p<0.01{ }^{* * *}: p<0.0001 ;{ }^{* \star * *}: p<0.00001$; data are not significant unless otherwise stated. 
A

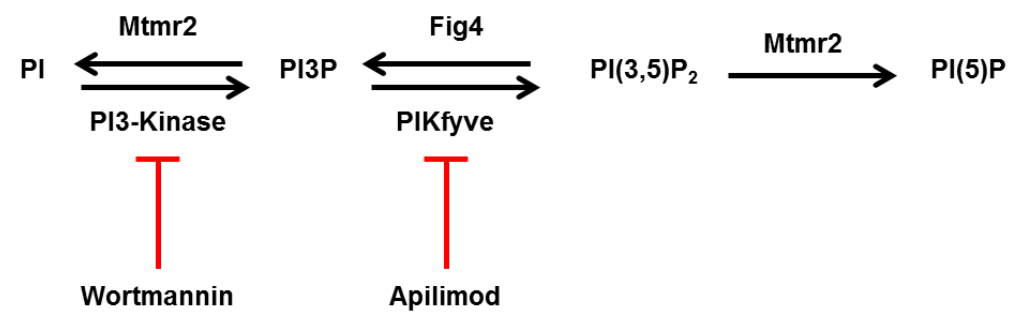

B

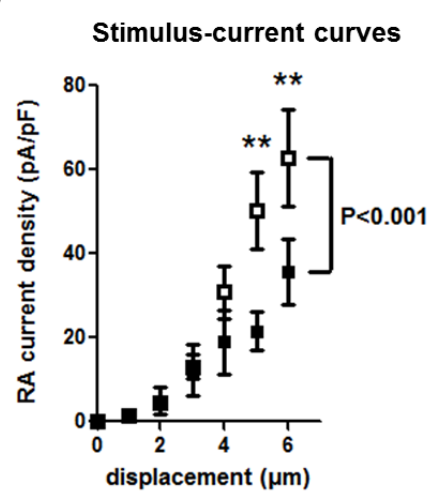

a Mtmr2 siRNA+ DMSO

- Mtmr2 siRNA + Apilimod
C

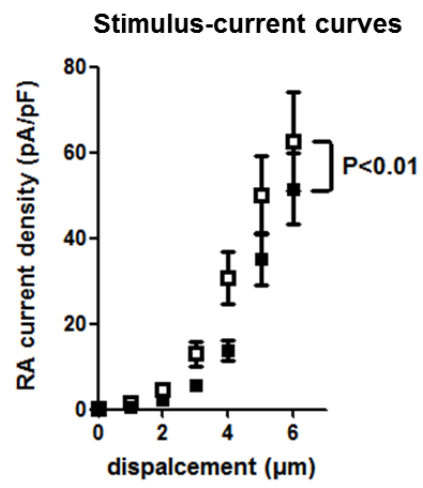

a Mtmr2 siRNA + DMSO

- Mtmr2 siRNA + Wortmannin
D

Activation threshold

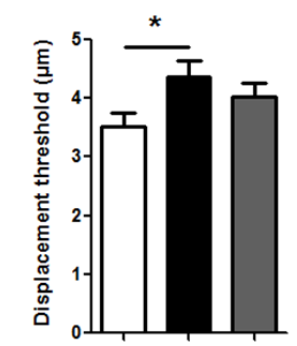

F Activation threshold

E Stimulus-current curves

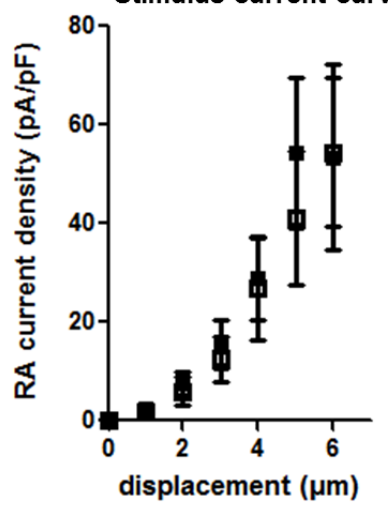

a + DMSO

- + Apilimod

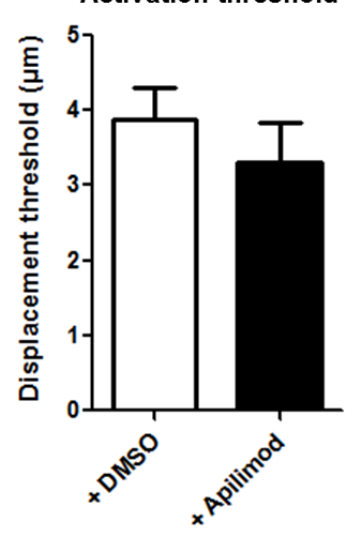

\subsubsection{Increasing levels of $\mathrm{PI}(3) \mathrm{P}$ and $\mathrm{PI}(3,5) \mathrm{P}_{2}$ in $\mathrm{DRG}$ neurons shows no effect}

From the scheme in Figure 27A, it can be inferred that loss of Mtmr2 increases $\mathrm{PI}(3,5) \mathrm{P}_{2}$. I attempted to recapitulate this effect using synthetic PIPs to artificially increase PIPs in DRG neurons. As described in the methods section (2.2.6), diC8 $\mathrm{PI}(3,5) \mathrm{P}_{2}$ or diC8 $\mathrm{PI}(3) \mathrm{P}$ (Echelon) was included in the intracellular recording solution during the measurement of MA currents (Dong et al., 2010). No significant effects were observed on RA-MA currents upon application of diC8 $\mathrm{PI}(3,5) \mathrm{P}_{2}$ or diC8 $\mathrm{PI}(3) \mathrm{P}$ to the DRG neurons (2way ANOVA with Holm-Sidak's multiple comparison test; Figure 28A). No significant differences were observed in the activation thresholds of the RA-MA currents either 
(Kruskal-Wallis test with Dunn's Multiple Comparison Test; Figure 28B). Of note, Dong and colleagues, used a similar approach to show the regulation of TRPML1 channels by $\mathrm{PI}(3,5) \mathrm{P}_{2}$ and $\mathrm{PI}(3) \mathrm{P}$ (Dong et al., 2010).

There could be several factors for the phospholipid addition not affecting RA-MA currents. The localization of the externally added PIPs may not correspond to the native cellular localization thus varying the action on targets. Additionally, the phosphatases either in the recording solution or inside the cell may break down the externally introduced PIPs before they can find their cellular target. Due to technical factors that may confound this data, a biochemical approach was used study Piezo2 and $\mathrm{PI}(3,5) \mathrm{P}_{2}$ interaction and the role Mtmr2 may play in it.

A

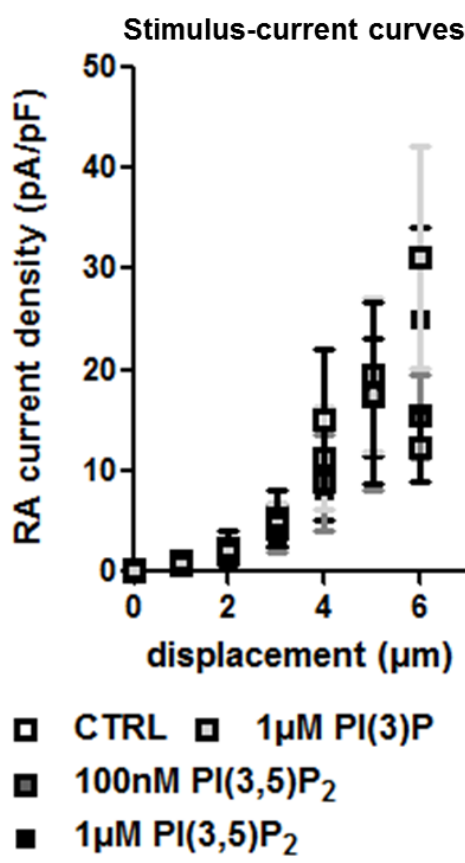

B

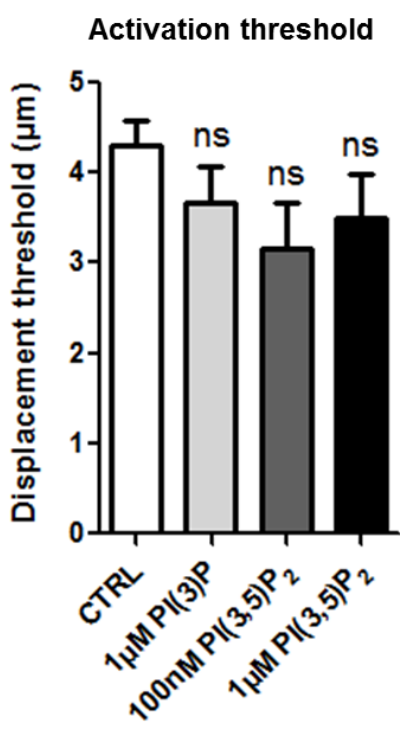

Figure 28: Synthetic PIP addition to DRG neurons does not affect RA-MA currents

Stimulus-current curves for RA-MA currents in DRG neurons upon addition of diC8 PIPs in intracellular solution (A) shows no difference between the applications of different concentrations of PIPs compared to control (CTRL: $\mathrm{n}=20-23$ neurons; $+1 \mu \mathrm{M} \mathrm{PI}(3) \mathrm{P}: \mathrm{n}=16$ neurons; $+100 \mathrm{nM} \mathrm{PI}(3,5) \mathrm{P}_{2}: \mathrm{n}=11-12$ neurons; $1 \mu \mathrm{M}$ $\mathrm{PI}(3,5) \mathrm{P}_{2}: \mathrm{n}=13-14$ neurons; ns; 2-way ANOVA with Holm-Sidak's multiple comparison test). Activation threshold upon application of diC8 PIPs intracellularly to DRG neurons (B) shows no significant differences compared to control (CTRL: $4.30 \pm 0.27 \mu \mathrm{m}, \mathrm{n}=23$ neurons; $+1 \mu \mathrm{M}$ PI(3)P: $3.67 \pm 0.40 \mu \mathrm{m}, \mathrm{n}=15$ neurons; $+100 \mathrm{nM} P \mathrm{Pl}(3,5) \mathrm{P}_{2}: 3.17 \pm 0.51 \mu \mathrm{m}, \mathrm{n}=12$ neurons; $1 \mu \mathrm{M} \mathrm{PI}(3,5) \mathrm{P}_{2}: 3.50 \pm 0.48 \mu \mathrm{m}, \mathrm{n}=14$ neurons; $\mathrm{ns}$; Kruskal-Wallis test with Dunn's Multiple Comparison Test). ${ }^{*}: p<0.05 ;{ }^{* *}: p<0.01 ;{ }^{* * *}: p<0.0001 ;{ }^{* * * *}$ : $p<0.00001$; data are not significant unless otherwise stated. 


\subsubsection{Piezo2 and Mtmr2 contain putative $\mathrm{PI}(3,5) \mathrm{P}_{2}$ binding motifs}

PIPs are known to bind to proteins through defined domains such as the FYVE domain, WD40 domain, PHD domain etc. or electrostatically to poly-basic regions with unstructured basic amino acid residues (Lysine or Arginine) (Dong et al., 2010; McCartney et al., 2014a). In order to glean if similar regions exist in the Piezo2 and Mtmr2 structures, I compared the structure of these proteins with a known $\mathrm{PI}(3,5) \mathrm{P}_{2}$ binding motif from the TRP channel, TRPML1. Dong and colleagues reported that $\mathrm{PI}(3,5) \mathrm{P}_{2}$ binds to the N-terminus region of TRML1 (Dong et al., 2010). Sequence comparison was done using $\mathrm{NCBI}$ protein blast (Protein [Internet]. Bethesda (MD): National Library of Medicine (US), National Center for Biotechnology Information).

Illustration in Figure 29 shows the results of this comparison. I found regions in both Piezo2 and Mtmr2 with considerable sequence similarity to the proposed $\mathrm{PI}(3,5) \mathrm{P}_{2}$ binding sequence of TRPML1. Worth noting is the interesting feature of these regions. The Piezo2 and Mtmr2 regions show similarity to mutually independent regions of the TRPML1 sequence and in effect combine to complete the $\mathrm{PI}(3,5) \mathrm{P}_{2}$ binding sequence of TRPML1.

In order to test, if the sequences identified in Piezo2 and Mtmr2 indeed bind to $\mathrm{PI}(3,5) \mathrm{P}_{2}$, the peptide-lipid binding assay was used as described in the methods section (2.2.5). Peptides of Piezo2 and Mtmr2 encompassing the regions mentioned in Figure 29 were purchased from GenScript (peptide sequences are provided in the supplementary table 5). Interestingly, the results show that both Piezo2 and Mtmr2 peptides bind to $\mathrm{PI}(3,5) \mathrm{P}_{2}$ (Figure 30A and $\mathbf{B}$ ). Besides $\mathrm{PI}(3,5) \mathrm{P}_{2}$, Mtmr2 also binds $\mathrm{PI}(3 \mathrm{P}), \mathrm{PI}(4) \mathrm{P}, \mathrm{PI}(5) \mathrm{P}$, and $\mathrm{PI}(4,5) \mathrm{P}_{2}$. Earlier studies have explored the binding of Mtmr2 to PIPs. It has been shown that the full length Mtmr2C417S and Mtmr2E276X (which contains only the PH-GRAM domain of the protein) binds to $\mathrm{PI}(3,5) \mathrm{P}_{2}, \mathrm{PI}(3,4,5) \mathrm{P}_{3}, \mathrm{PI}(4) \mathrm{P}$, and $\mathrm{PI}(5) \mathrm{P}$ (Berger et al., 2003). Another study also showed that Mtmr2 can bind to $\mathrm{PI}(3) \mathrm{P}$ and $\mathrm{PI}(3,5) \mathrm{P}_{2}$ (Begley et al., 2006).

In the case of Piezo2 peptide besides $\mathrm{PI}(3,5) \mathrm{P}_{2}$, it also bound to $\mathrm{PI}(4,5) \mathrm{P}_{2}$. This is an interesting observation because in 2015 Borbiro and colleagues showed that TRPV1 modulates Piezo2 currents through $\mathrm{PI}(4,5) \mathrm{P}_{2}$ depletion, though the $\mathrm{PI}(4,5) \mathrm{P}_{2}$ binding region of Piezo2 was not identified in the study (Borbiro et al., 2015).

In order to test the relevance of the basic amino acids to the Piezo2- $\mathrm{PI}(3,5) \mathrm{P}_{2}$ binding, I generated a $3 Q$ mutant of the Piezo2 peptide (Figure 29; Piezo2 3Q mutant; QQILQYFWMS). The basic amino acid residues in the Piezo2 peptide were changed to neutral Glutamine (Q). Remarkably, the Piezo2 $3 \mathrm{Q}$ mutant does not bind to $\mathrm{PI}(3,5) \mathrm{P}_{2}$ (Figure 30C). This observation indicated, that the basic residues in the Piezo2 peptide sequence are essential for the binding to $\mathrm{PI}(3,5) \mathrm{P}_{2}$. Considering the interesting bi-partite 
arrangement of the Piezo2 and Mtmr2 peptide sequences as shown in Figure 29, I asked if the Piezo2 and Mtmr2 peptide bind to each other. For this the peptide-peptide overlay assay was used as described in the methods section (2.2.5). As shown in Figure 30D, the Mtmr2 peptide binds to the Piezo2 peptide, but not to the Piezo2 3Q mutant described earlier. Another Piezo2 peptide (Piezo2X) was generated encompassing a region similar to the Piezo2 peptide. It contained similar basic amino acid residues as the Piezo peptide, only arranged differently (LWMIRNRRKYAMISSP). The fact that the Mtmr2 peptide fails to bind to Piezo2 $X$ peptide indicates that the Mtmr2-Piezo2 interaction not only requires the presence of basic amino acids but also depends on the exact amino acid sequence. Additional controls including TRPA1 and TRPV1 peptides were all negative (data not shown). This data points to a possible tripartite interaction between Piezo2-Mtmr2 and $\mathrm{PI}(3,5) \mathrm{P}_{2}$. The exact structural make up of this interaction remains to be studied. It is important to note here that the peptide regions defined here may not be the only $\mathrm{PI}(3,5) \mathrm{P}_{2}$ binding regions in Piezo2 and Mtmr2. In fact, a study describing the Mtmr2- $\mathrm{PI}(3,5) \mathrm{P}_{2}$ binding pocket identified other regions in the protein that can bind to $\mathrm{PI}(3,5) \mathrm{P}_{2}$ (Begley et al., 2006). Of note, in order to study the relevance of the region shown above in Piezo2 function, I designed a Piezo2 3Q mutant construct (the basic amino acids in the Piezo2 region described in Figure 29 were changed to Glutamine $(Q))$. The mutant construct failed to express as a functional channel in HEK293 cells and hence RA-MA currents could not be measured.

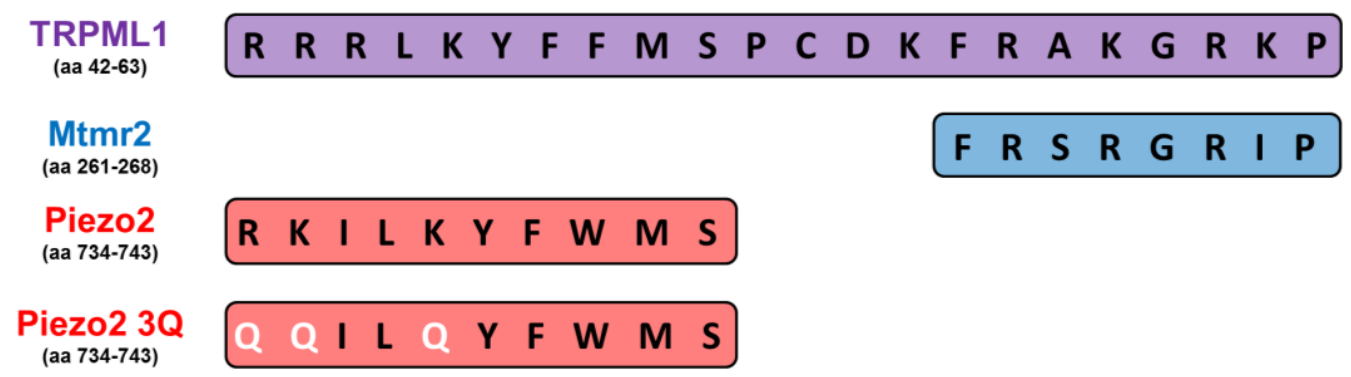

Figure 29: PIP $_{2}$ binding sequences in Piezo2, Mtmr2 and TRPML1

Schematic illustration of the protein sequences which show the $\mathrm{PI}(3,5) \mathrm{P}_{2}$ binding regions of the TRPML1, Mtmr2 and Piezo2. TRPML1 binds to $\mathrm{PI}(3,5) \mathrm{P}_{2}$ through its $\mathrm{N}$-terminal region as described in Dong et al. (2010). Mtmr2 and Piezo2 sequences that have significant sequence similarities were identified using NCBI protein Blast (Protein [Internet]. Bethesda (MD): National Library of Medicine (US), National Center for Biotechnology Information). Additionally, Piezo2 $3 \mathrm{Q}$ peptide is also shown, in which the basic amino acids have been mutated to neutral $(Q$, Glutamine) residues. 


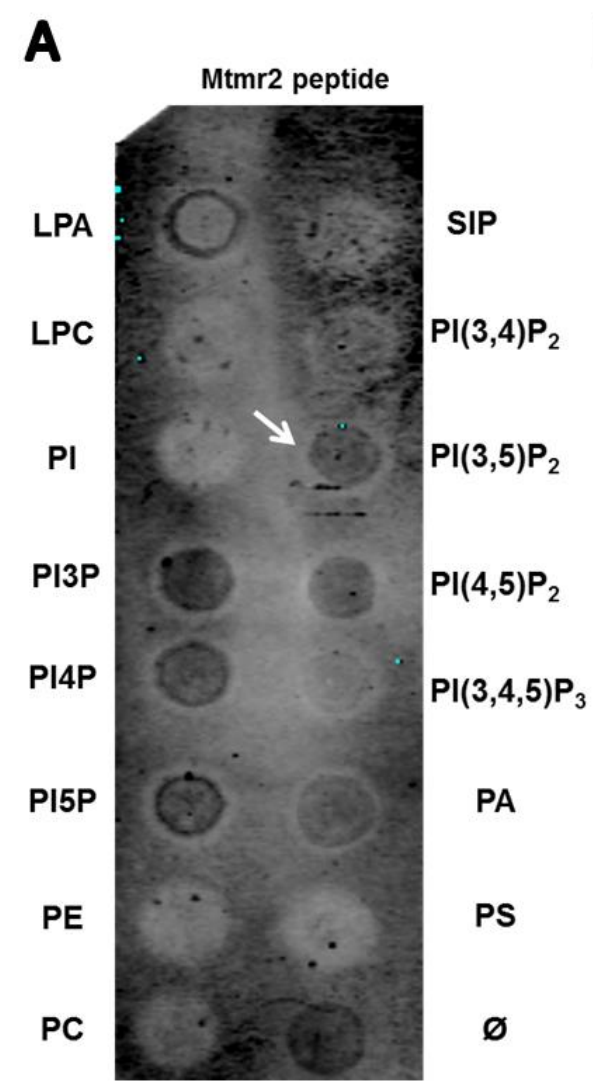

B

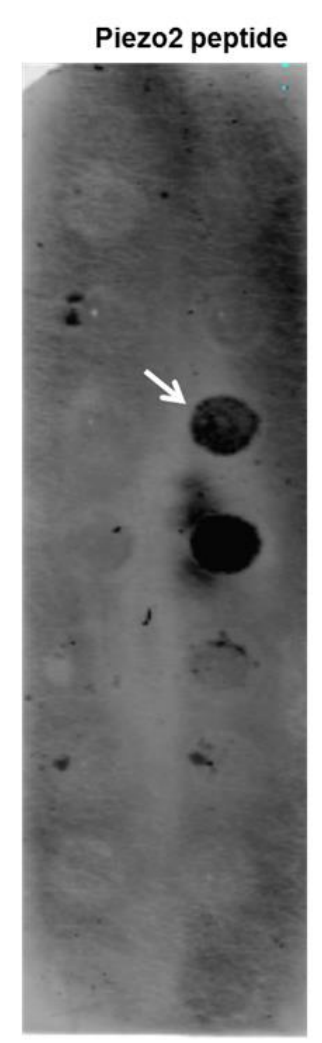

C

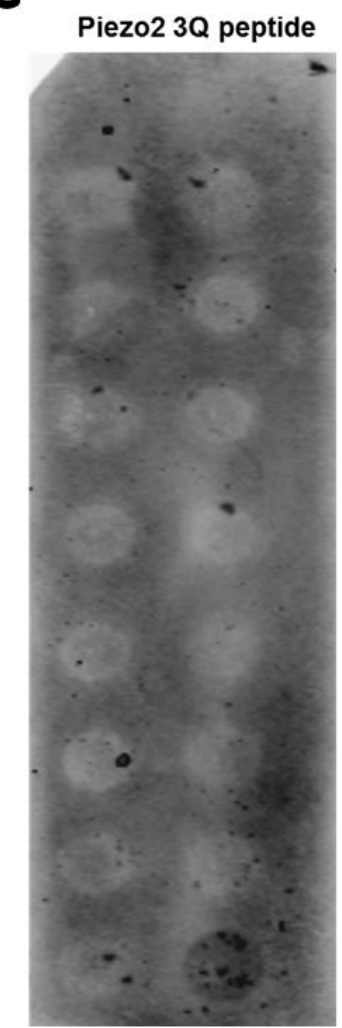

D

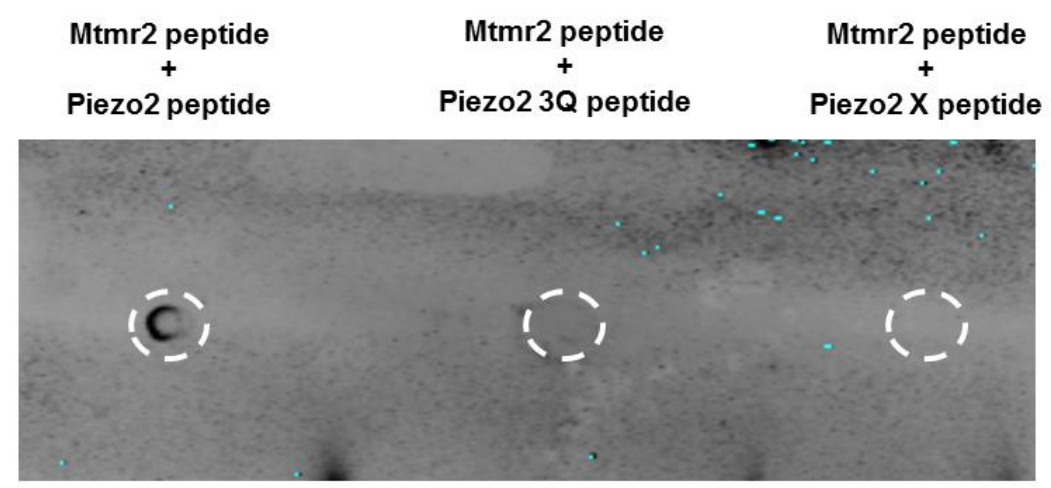

Figure 30: Piezo2 and Mtmr2 bind to $\mathrm{PI}(3,5) \mathrm{P}_{2}$

Representative immunoblot of Mtmr2 peptide on PIP strip (A) shows that Mtmr2 binds to $\mathrm{PI}(3,5) \mathrm{P}_{2}$ (arrow). Mtmr2 also binds to $\mathrm{PI}(4,5) \mathrm{P}_{2}, \mathrm{PI}(3) \mathrm{P}, \mathrm{PI}(4) \mathrm{P}$ and $\mathrm{PI}(5) \mathrm{P}$. Representative immunoblot of PIP strip bound to Piezo2 peptide (B) shows that Piezo2 binds to $\mathrm{PI}(3,5) \mathrm{P}_{2}$ (arrow) and $\mathrm{PI}(4,5) \mathrm{P}_{2}$. Representative immunoblot of PIP strip bound to Piezo2 $3 Q$ peptide (C). The $3 Q$ peptide contains the basic amino acids changed to neutral glutamine (Q). Piezo2 $3 \mathrm{Q}$ does not bind to $\mathrm{PI}(3,5) \mathrm{P}_{2}$. Representative dot blot (D) shows Piezo2 and Mtmr2 peptides bind to each other. As control, Mtmr2 does not bind to the Piezo2 $3 Q$ peptide or to the Piezo2 $X$ peptide. (Piezo2 $X$ peptide was generated encompassing a region similar to the Piezo2 peptide containing basic amino acids; for sequence information please refer to Supplementary Table 5). Dotted lines indicate the region of peptide application. 


\subsubsection{Physiological manipulation of PIP levels in DRG neurons}

So far the experiments point to a role for $\mathrm{PI}(3,5) \mathrm{P}_{2}$, in the Piezo2-Mtmr2 interaction and that the three may form a tripartite interaction complex. As described earlier, $\mathrm{PI}(3,5) \mathrm{P}_{2}$ is a low abundance phosphatidylinositol whose levels change in response to cellular stress. Osmotic shock in eukaryotic cells was shown to transiently increase the cellular levels of $\mathrm{PI}(3,5) \mathrm{P}_{2}$. The increase was shown to last for 10 minutes and then drop to baseline immediately (McCartney et al., 2014a). Based on this evidence, osmotic stress was used in DRG neurons to further analyze the interplay of $\mathrm{PIP}_{2}$ levels and Piezo2 function. It has already been shown that Piezo2 RA-MA currents in DRG neurons are modulated by osmotic stress (Jia et al., 2016), without affecting single channel properties. The study showed that osmotic swelling causes potentiation of RA-MA currents in rat DRG neurons due to changes in the static plasma membrane tension.

\section{Extracellular hypotonic stress potentiates Piezo2 mediated current magnitude in DRG} neurons

I tested this osmotic stress paradigm in DRG cultures from mice and found that extracellularly applied hypotonic solution indeed caused potentiation of the Piezo2 RAMA currents. The RA-MA current magnitude in hypotonic extracellular solution treated cells was significantly higher than control (2-way ANOVA with Holm-Sidak's multiple comparison test; Figure 31A). Strikingly, Apilimod application partially attenuated the potentiation of RA-MA currents (2-way ANOVA with Holm-Sidak's multiple comparison test; Figure 31B). Furthermore, the activation threshold of the RA-MA currents was significantly decreased upon treatment with hypotonic extracellular solution (Green bar; 1-way ANOVA with Uncorrected Fisher's LSD; Figure 31C). This decrease of threshold could be rescued by the application of Apilimod (Red bar; 1-way ANOVA with Uncorrected Fisher's LSD; Figure 31C). In the above mentioned study Jia and colleagues also reported that prolonged exposure of DRG neurons to hypotonic stress causes a slowing down of the inactivation (Jia et al., 2016). In order to ensure that the MA currents measured here were of the RA-MA category, I tested the inactivation time constant of the currents. The inactivation time constant $(\tau)$ was unchanged upon application of hypotonic stress compared to isotonic extracellular solution (Isotonic extra solution: $2.93 \pm 0.41 \mathrm{~ms}, \mathrm{n}=22$ neurons; Hypotonic extra solution: $2.88 \pm 0.24 \mathrm{~ms}, \mathrm{n}=24$ neurons; ns; Mann Whitney test). 
Apilimod reverses the hypersensitivity caused by hypotonic saline injection in mouse paw

Remarkably, the effect of hypotonic stress in vitro and its rescue by Apilimod application could be recapitulated in behavior experiments in mice. Responses to innocuous mechanical stimuli are a commonly used measure to study Piezo2 function in vivo (Eijkelkamp et al., 2013; Poole et al., 2014; Ranade et al., 2014). Mouse paw is stimulated using a mechanical filament and the force which elicits a withdrawal response is measured. Lower forces required to elicit withdrawal response indicates hypersensitivity of the paw. Unilateral injection of hypotonic saline (0.5X dPBS) to the mouse plantar hind paw surface leads to a hypersensitivity to innocuous mechanical stimuli (please refer to methods section for details). Quantification of mechanical threshold (force, g) illustrates the tactile allodynia induced upon injection of hypotonic saline when comparing ipsilateral (injected paw; green triangle) versus contralateral (uninjected paw; green circle) (2-way ANOVA for repeated measures followed by HolmSidak's multiple comparison test; Figure 31D). Additionally, in line with the data shown in Figure 31B and C, Apilimod co-injection with hypotonic saline to the hind paw plantar surface, significantly increases the hind paw mechanical threshold indicating an alleviation of the tactile allodynia (2-way ANOVA for repeated measures followed by Holm-Sidak's multiple comparison test; Figure 31D). It is relevant to note here that Apilimod alone injected in mouse paw does not influence mechanical sensitivity (data not shown), which is in line with the in vitro data shown in Figure 27E and F. Based on these data it can be said that $\mathrm{PI}(3,5) \mathrm{P}_{2}$ has a role to play in modulating mechanical sensitivity both in vitro and in vivo. The extent to which this regulation is mediated by Piezo2 remains to be tested. Other proteins like TRPV4 through its role as an osmo-sensor may be affected by the application of hypotonic stress (Jia et al., 2016; Lechner et al., 2011; Liu et al., 2007; Quallo et al., 2015). Interestingly in vitro, Jia and colleagues showed that the Piezo2 potentiation in DRG neurons upon exposure to osmotic stress is independent of TRPV4 function (Jia et al., 2016). 
Figure 31: Apilimod rescues potentiation of RA-MA currents and mechanical allodynia, caused by osmotic stress

Stimulus-current curves of RA-MA currents in DRG neurons upon exposure to hypotonic extracellular stress (A) shows a significant potentiation of RA-MA currents compared to control (Isotonic extracellular solution: $n=23-29$ neurons; Hypotonic extracellular solution: $n=26-29$ neurons; 2-way ANOVA reports that hypotonic extracellular stress has a significant effect $(P<0.0001)$ on RA-MA Piezo2 currents; Holm-Sidak's multiple comparison test was used to compare both conditions at individual stimulus magnitudes, results are represented on the graph with '*'). (B) Apilimod application alleviates the RA-MA potentiation caused by the hypotonic stress (Isotonic extracellular solution: $n=23-29$ neurons; Hypotonic extracellular solution: $n=26-29$ neurons (same datasets as above); Hypotonic extracellular solution + Apilimod: $n=14-15$ neurons). 2-way ANOVA suggests that Apilimod can alleviate the Piezo2 RA-MA potentiation $(P<0.05)$. Holm-Sidak's multiple comparison test was used to compare both conditions at individual stimulus magnitudes, results are represented on the graph with "*'. Activation threshold of RA-MA currents under hypotonic extracellular stress (C) shows a pattern consistent with the stimulus-current curves (Extracellular isotonic solution: $3.74 \pm$ $0.29 \mu \mathrm{m}, \mathrm{n}=23$ neurons; Extracellular hypotonic solution: $2.90 \pm 0.26 \mu \mathrm{m}, \mathrm{n}=29$ neurons; Extracellular hypotonic solution + Apilimod: $3.80 \pm 0.35, \mathrm{n}=15$ neurons; 1 -way ANOVA with Uncorrected Fisher's LSD). *: $p<0.05 ;{ }^{* *}: p<0.01 ;{ }^{* * *}: p<0.0001 ;{ }^{* * *}: p<0.00001$; data are not significant unless otherwise stated. Behavioral data corroborate the above shown in vitro data. Quantification of mouse hind paw withdrawal threshold (force, g) after unilateral injection of hypotonic saline co-injected with vehicle (DMSO) or Apilimod (D). Reduction of withdrawal threshold of injected (ipsilateral, ipsi) paw compared to non-injected (contralateral, contra) paw indicates tactile allodynia i.e. lowered mechanical thresholds ( $\mathrm{N}=10$ animals per condition). Apilimod co-application significantly attenuates tactile allodynia (Hypotonic saline + DMSO ipsilateral; Hypotonic saline + Apilimod ipsilateral; $\mathrm{N}=10$ animals per condition; ${ }^{*} \mathrm{p}<0.05,{ }^{* *} \mathrm{p}<0.01$ black font; 2-way ANOVA for repeated measures followed by Holm-Sidak's multiple comparison test). Comparing corresponding ipsilateral versus contralateral paws within one condition (2-way ANOVA for repeated measures followed by Holm-Sidak's multiple comparisons test; \#p<0.05, \#\#p<0.001; Hypotonic + Vehicle, green colour; Hypotonic + Apilimod, red colour). All measurements were made in three time intervals 0-30 minutes, 30-60 minutes and 60-90 minutes after injection. 
A

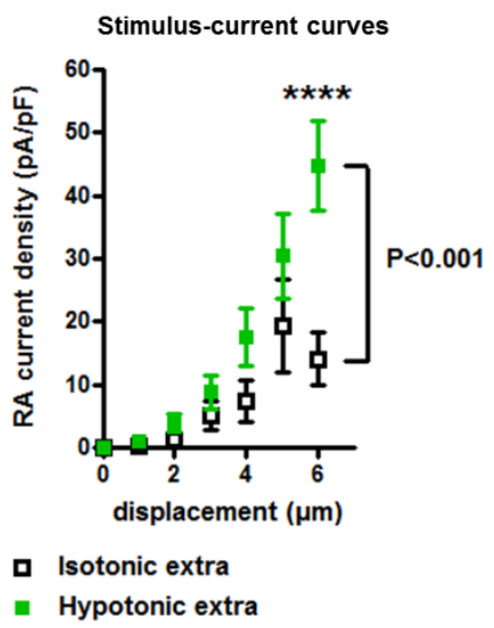

C

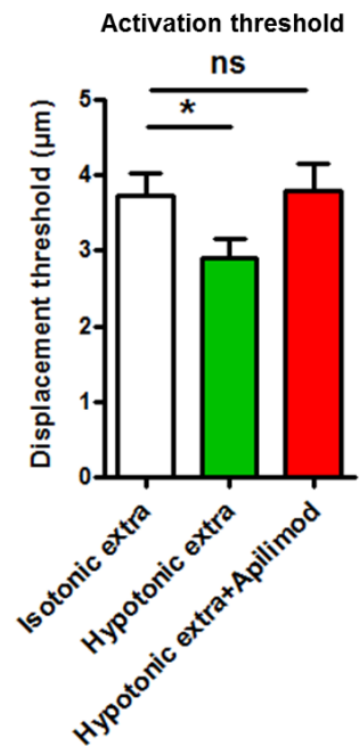

B

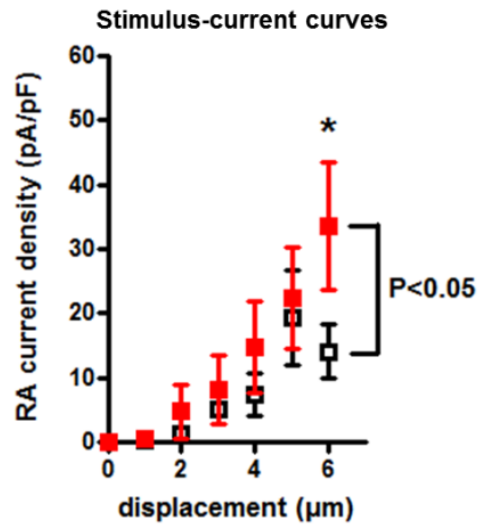

口 Isotonic extra

- Hypotonic extra+Apilimod
D

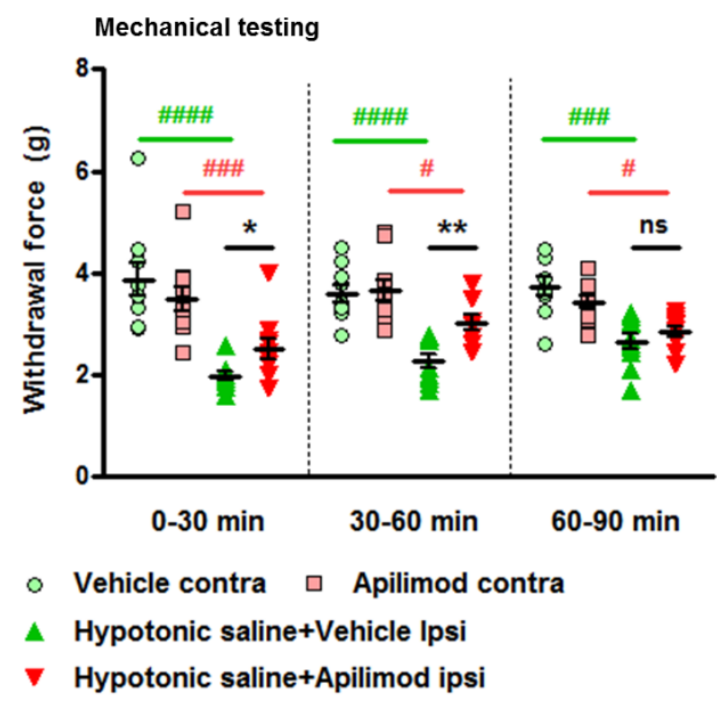

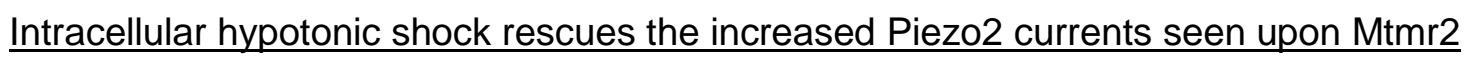
knockdown

Additional proof that $\mathrm{PI}(3,5) \mathrm{P}_{2}$ is indeed a relevant factor for modulating RA-MA currents came from re-examining the Mtmr2 knockdown. Mtmr2 knockdown in DRG neurons would be expected to increase the cellular levels of $\mathrm{PI}(3,5) \mathrm{P}_{2}$ as can be appreciated from the scheme in Figure 26A. From the literature and previous experiments it is known that osmotic stress can manipulate $\mathrm{PI}(3,5) \mathrm{P}_{2}$ levels (McCartney et al., 2014a). 
In lieu of these observations, I tested if the application of osmotic stress (intracellular hypotonicity, which would be expected to decrease $\mathrm{PI}(3,5) \mathrm{P}_{2}$ levels) would cause reversal of the RA-MA phenotype observed upon Mtmr2 knockdown (Figure 24). Strikingly, the application of hypotonic intracellular solution to Mtmr2 knockdown neurons indeed reduces the RA-MA current magnitude compared to application of isotonic intracellular solution (2-way ANOVA followed by Holm-Sidak's test; Figure 32A). Activation threshold of the RA-MA currents remained unchanged upon this treatment (Mann Whitney test; Figure 32B). This result reinforces the idea that $\mathrm{PI}(3,5) \mathrm{P}_{2}$ plays an important part in the Mtmr2-Piezo2 interaction and may also be involved in direct modulation of Piezo2.

A

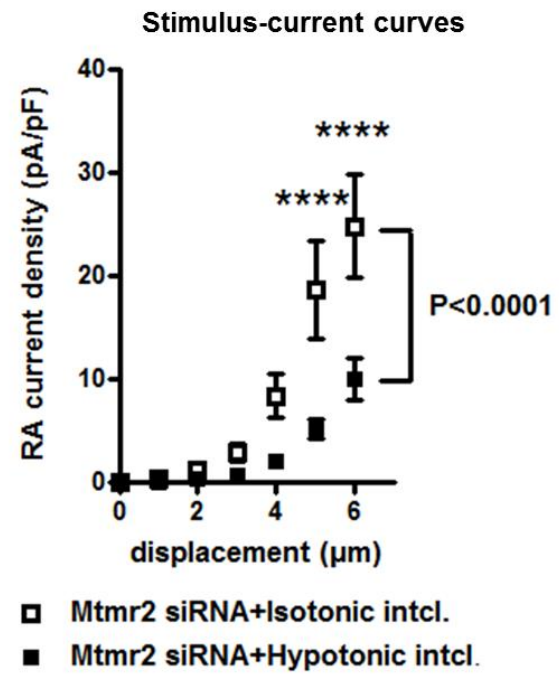

B

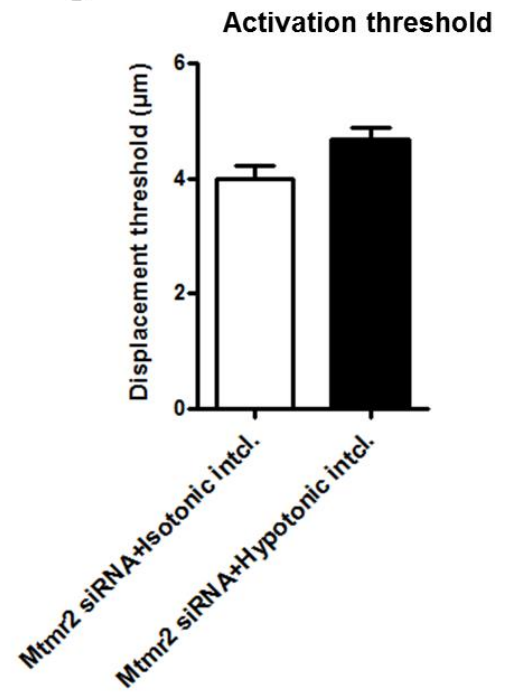

Figure 32: Hypotonic intracellular stress rescues RA-MA potentiation upon loss of Mtmr2

Stimulus-current curves of RA-MA currents in DRG neurons treated with Mtmr2 siRNA upon exposure to hypotonic extracellular stress (A). As shown in Figure 24, treatment of DRG neurons with Mtrm2 siRNA causes potentiation of Piezo2 RA-MA currents. Application of intracellular hypotonic stress to the Mtmr2 siRNA treated neurons, caused a significant decrease in the potentiated RA-MA currents (Mtmr2 siRNA + isotonic intracellular solution: $n=30$ neurons; Mtmr2 siRNA + hypotonic extracellular solution: $n=28$ neurons; 2-way ANOVA suggested a significant $(P<0.0001)$ effect on the RA-MA currents. Holm-Sidak's multiple comparison test was performed to compare both conditions at individual stimulus magnitude, $p$ values are indicated on the graph with '*'). (B) Activation threshold of RA-MA currents was unchanged upon application of the hypotonic stress (Mtmr2 siRNA + isotonic intracellular solution: $4.00 \pm 0.23 \mu \mathrm{m}, \mathrm{n}=26$ neurons; Mtmr2 siRNA + hypotonic intracellular solution: $4.68 \pm 0.20 \mu \mathrm{m}, \mathrm{n}=19$ neurons; ns; Mann Whitney test). ${ }^{*}: p<0.05 ;{ }^{* *}: p<0.01 ;{ }^{* * *}: p<0.0001 ;{ }^{* * *}: p<0.00001$; data are not significant unless otherwise stated. 


\subsection{Summary of part 2}

$>$ Myotubularin related protein (Mtmr2) was identified as a high probability interactor of Piezo2

$>$ Co-expression of Piezo2 with Mtmr2 suppresses RA-MA (Piezo2) currents in HEK293 cells.

> Upon overexpression in DRG neurons, Mtmr2 suppresses native RA-MA currents.

$>$ Loss of Mtmr2 in DRG neurons potentiates Piezo2 mediated RA-MA currents specifically

$>$ Mtmr2 phosphatase activity is required for the modulation of RA-MA currents seen in HEK293 cells

$>\mathrm{Pl}(3,5) \mathrm{P}_{2}$ ( and to a lesser extent $\left.\mathrm{PI}(3) \mathrm{P}\right)$ is involved in the modulation of Piezo2 currents

$>$ Basic amino acid rich peptide sequences in Piezo2 and Mtmr2 can bind $\mathrm{PI}(3,5) \mathrm{P}_{2}$

$>$ Hypotonic cellular stress potentiates Piezo2 through changes in cellular levels of $\mathrm{PI}(3,5) \mathrm{P}_{2}$

> Hypotonic stress induced mechanical allodynia in mouse paw, also mediated via changes in $\mathrm{PI}(3,5) \mathrm{P}_{2}$ levels 


\section{DISCUSSION}

\subsection{Relevance of studying Piezo2 protein complexes}

Piezo2 is the bona fide MA channel in mouse somatosensory neurons (Coste et al., 2010; Coste et al., 2012). It is essential for light touch and proprioception in mouse (Florez-Paz et al., 2016; Ranade et al., 2014; Woo et al., 2015). There is also accumulating evidence for the role of Piezo2 in mechanical allodynia and hyperalgesia (Dubin et al., 2012; Eijkelkamp et al., 2013; Ferrari et al., 2015; Lolignier et al., 2014). Despite these advances in the understanding of Piezo2, the structure and regulation of the channel remains uncharacterized. Recent studies have explored the structure of Piezo1 (the other member of the mammalian Piezo family) (Coste et al., 2015; Ge et al., 2015; Wu et al., 2016a) and an understanding of the Piezo2 structure may soon follow. Yet the regulation of the Piezo2 ion channel is hardly understood. Figure 4 illustrates the regulators/interactors of Piezo2 that have been identified so far. Stoml3 (Stomatin-like protein 3) directly interacts with Piezo2 and modulates its activity by influencing local membrane properties (Poole et al., 2014; Qi et al., 2015). Loss of Stoml3 in mice causes a reduction in the tactile discrimination performance and a loss of mechanical allodynia upon chronic constriction injury (Wetzel et al., 2007). Another known regulator of Piezo2 is transcription factor, Runx1. Loss of Runx1 from a subset of somatosensory neurons causes a decrease in the number of Piezo2 positive small diameter neurons (Lou et al., 2013). Besides this, CamKII and Kv1.1 are regulators of mouse somatosensory mechanotransduction which have not yet been shown to be directly linked to Piezo2 (Hao et al., 2013; Yu et al., 2015).

Considering that the sense of touch is a combination of several modalities, it requires the components of the system to have a large and varied stimulus-response range. Some of these variations are generated by anatomical structures including the HTMRs and LTMRs (Abraira and Ginty, 2013), yet I believe that some of this variation must arise from the modulation of molecular components including Piezo2. Ion channels often exist as part of multi-protein complexes which are involved in their transport, regulation etc. (Levitan, 2006). Our group set out to identify if Piezo2 in somatosensory DRG neurons also exists as part of such a complex and used a mass-spectrometry based interactomics approach to identify potential components of said complex (Narayanan et al., 2016). 


\subsubsection{Identified proteins and possible relevance to Piezo2 function}

Through the screen we identified 36 potential interactors of Piezo2 in DRG neurons (Figure 6B; Narayanan et al. (2016)). These identified proteins are involved in protein transport, cellular localization, protein autophosphorylation, cell cycle regulation etc. Some of the proteins identified in the screen have already been implicated in somatosensation. Potassium channel subunits Kcna1 and Kcna2 were implicated in mechanotransduction. Interestingly, Kcna1 $\left(\mathrm{K}_{\mathrm{v}} 1.1\right)$ displays MA currents which regulates mechanical sensitivity of C-HTMRs and A $\beta$-mechanoreceptors (Hao et al., 2013). Another protein identified on the screen is the calmodulin-dependent protein kinase II (CamKII). CamKII is involved in calcium-mediated cell signaling and activity dependent changes in neurons (Hudmon and Schulman, 2002). CamKII has recently been implicated in the adaptation of low-threshold A $\beta$-mechanoreceptors (Yu et al., 2015). Whether these two proteins are direct modulators of Piezo2 remains to be studied.

\subsubsection{Challenges and limitations of this approach}

Our study in 2016 is the first to systematically identify the interactors of Piezo2 in mouse somatosensory neurons (Narayanan et al., 2016). Certain aspects of this study merit discussion. An important one is the use of the DRG tissue (where cell bodies of the DRG neurons lie) to identify the Piezo2 interactors. It is implicit that Piezo2 expressed in the peripheral tissue (e.g. skin) would be more ideal to study its role and regulation in fine touch. This was considered at the conceptualization of the study and various factors prompted the study to be carried out in the tissue containing the cell bodies. Firstly, technical difficulties make the study of protein complexes in peripheral nerve endings, complicated. The peripheral nerve endings are very sparsely located in tissues such as skin and further Piezo2 is only expressed in a subset of these nerve endings (Abraira and Ginty, 2013; Ranade et al., 2014; Woo et al., 2014). It would therefore be methodologically challenging to obtain enough material for an immunoprecipitation based analysis. Secondly, it is known that Piezo2 is expressed both peripheral nerve endings and DRG neurons (Coste et al., 2010; Ranade et al., 2014). Of note, Piezo2 was first identified as the MA channel in murine somatosensation, in the cells bodies of DRG neurons (Coste et al., 2010). Additionally several studies have shown that manipulating Piezo2 in sensory neuron cell bodies can affect Piezo2-mediated behaviors in vivo (Eijkelkamp et al., 2013; Ranade et al., 2014) indicating that properties of Piezo2 in the cell bodies are reflective of its role in tactile behavior. Therefore it is expected that the components required for the function of Piezo2 are present at both cell body and peripheral levels. Despite this, it cannot be ruled out that there may be interactors of 
Piezo2 that may be specific to cell bodies or peripheral nerve endings. One way to address this issue, is to orthogonally validate the interactomics approach using in vitro functional studies and characterization of the identified interactors in vivo and study their role in regulating Piezo2 mediated behaviors. This can prove useful to discriminate between interactors that may be cell body specific and interactors that play a role in Piezo2 mediated behaviors. Therefore, an interactomics screen in the cell bodies has the potential to identify meaningful interactors of mechano-gated channel, Piezo2.

Although our interactomics screen as mentioned in the last section was capable of identifying biologically significant interactors of Piezo2, it is important to note that the method has its limitations. Though the conditions of immunoprecipitation in DRG neurons were as close as biochemically possible to the in vivo situation, this approach may miss transient or weak interactions. Hence the list of interactors identified by us may not represent the complete interactome of Piezo2 (Narayanan et al., 2016). One key Piezo2 interactor that we did not find on our screen was Stoml3. As has been mentioned several times in this study, Stoml3 modulates Piezo2 activity in DRG neurons and regulates Piezo2 mediated behaviors in mice (Poole et al., 2014; Qi et al., 2015; Wetzel et al., 2007). There could be several reasons for the failure of our screen to identify Stoml3. Firstly, Stoml3 may transiently regulate Piezo2, making its identification through immunoprecipitation difficult. Furthermore, originally Piezo2-Stoml3 direct binding has only been seen in HEK293 cells (Poole et al., 2014). It is therefore possible that in DRG neurons the proteins do not interact directly but rather through other intermediates. Lastly, the cellular abundance or stoichiometry of proteins may play an important role in their identification through immunoprecipitation. This may explain the difference between an overexpression system like HEK293 cells and native system of DRG neurons. In conclusion, though the interactomics screen provides a robust resource for the exploration of Piezo2 regulation (Narayanan et al., 2016), it is important to keep in mind that this in no way represents the complete Piezo2 interactome.

\subsection{Identification of Pericentrin (Pcnt) as a Piezo2 interactor in DRG neurons}

After the identification of potential Piezo2 interactors, the first step was the orthogonal validation of the interactors to show that the screen indeed identified meaningful interactors of Piezo2. Of the identified components of the putative Piezo2 interactome, Pcnt came up as an unlikely interactor of Piezo2. It is a protein known for its role in cell cycle regulation and organization of the microtubule organizing complex (MTOC) and has not been extensively studied in the context of sensory systems (Delaval and Doxsey, 2010). Pcnt was chosen for the orthogonal validation of the screen for two reasons; 
firstly, considering it was an unlikely interactor of Piezo2, a validation of its interaction with Piezo2 would highlight the robustness of our interactomics screen; and secondly, a study in Drosophila showed that loss of Pcnt homolog (d-PLP) in flies caused mechanosensory deficits in the fly (Martinez-Campos et al., 2004). This indicated a possible link for Pcnt to somatosensory mechanotransduction. Based on these factors I proceeded to characterize the role of Pcnt in Piezo2 function.

The data obtained in this study indicate that Piezo2 and Pcnt are expressed in close apposition in the somatosensory endings in the skin and in DRG neuron cell bodies. The identification of Pcnt in the somatosensory endings came as a surprise, since Pcnt has never been reported in similar structures and its described roles so far do not anticipate such localization (Delaval and Doxsey, 2010). Due to limitations of experimental techniques we could not study this localization in more detail (Narayanan et al., 2016). Therefore the function of this close apposition of Pcnt with Piezo2 in the nerve endings remains unknown. It would be interesting to use high resolution microscopy to gain a better understanding of these peripheral endings. It is relevant to note here that the cutaneous endings (LTMRs) are engulfed by terminal Schwann cells (Li et al., 2011; Shenton et al., 2014). It therefore cannot be excluded that the Pcnt immunolabel seen in these structures arises in the Schwann cells (as is also hinted from our own data showing apposition of Pcnt immunolabel with Schwann cell marker, S100). Interestingly, Schwann cells have been shown to form cellular junctions with the terminal endings which could be relevant for cellular cross-talk or regulation (Kaidoh and Inoué, 2000). Hence, if the Pcnt localized to the Schwann cells could still be a regulator of neuronal properties.

In the cell bodies of DRG neurons, loss of Pcnt potentiates Piezo2 RA-MA currents with remarkable specificity. In addition I also found the level of membrane Piezo2 was increased upon loss of Pcnt. An increase in the number of Piezo2 channels on the membrane could explain the increased magnitude of MA currents. It remains to be seen how Pcnt contributes to increase in membrane expression of the channel.

Interestingly, Pcnt besides its role in cell cycle regulation is also known to be part of the microtubule organizing complex (MTOC) (Chen et al., 2004; Doxsey et al., 1994). Based on this knowledge, regulation of microtubule based transport of Piezo2 to the membrane was explored as a potential mechanism for Piezo2-Pcnt interaction. Contrary to expectations, Nocodazole, an inhibitor of microtubule stability did not affect Piezo2 mediated currents (Dammermann, 2002). This could be either because Piezo2 transport to the plasma membrane is not mediated by microtubules or could hint to multiple mechanism for Piezo2 transport which could have compensatory functions. It is known that stability of actin cytoskeleton plays a crucial role in Piezo2 function (Jia et al., 2016). Whether it has a role in localization of Piezo2 to plasma membrane remains to be tested. 
Notably, the role of cytoplasmic Pcnt has not been elucidated so far. As we and others have observed (Chen et al., 2014; Jurczyk et al., 2010; Miyoshi et al., 2009; Mühlhans and GießI, 2012; Narayanan et al., 2016), besides its centrosomal and MTOC localization, Pcnt is also expressed in the cytoplasm in certain cell types. According to immunostaining data (Figure 13B and C), nucleofection of DRG neurons with Pcnt siRNA, decreases Pcnt expression in both the centrosomal and cytoplasm. Therefore it is possible that the potentiation of Piezo2 currents seen here could be due to loss of cytoplasmic Pcnt.

Interestingly, one study found Pcnt enriched in the secretory vesicles in the cytoplasm of pancreatic $\beta$-cells. RNAi mediated knockdown of Pcnt in these cells resulted in hypersecretion of these vesicles (Jurczyk et al., 2010). This result presents the idea that cytoplasmic Pcnt may be involved in regulation of secretory vesicles and thereby Piezo2 localization to the plasma membrane. This hypothesis can be tested by treating Pcnt knockdown DRG neurons with inhibitors of exocytosis. If loss of Pcnt indeed dysregulates vesicle exocytosis, the inhibitor would be expected to rescue the effect.

Of note, our interactomics screen also identified pericentriolar material 1 (PCM1), an already known interactor of Pcnt (Dammermann, 2002; Li et al., 2001). It is therefore possible that Pcnt and PCM1 form a complex that controls Piezo2 function. It would be interesting to explore this multi-protein interaction and its relevance to Piezo2 function.

In order to characterize the Piezo2-Pcnt interaction and understand its mechanism, a somatosensory system specific inducible knockdown of Pcnt would be a valuable tool. It is important to note here that due to the role of Pcnt in cell cycle regulation, a systemic knockdown of Pcnt is prenatally lethal (Chen et al., 2014). Therefore a knockdown specific to the somatosensory neurons would help analyze the relevance of Pcnt in Piezo2 mediated behaviors in vivo.

Nonetheless, identification of Pcnt as a meaningful interactor of Piezo2 in DRG neurons reinforces the validity of our interactomics approach and opens the possibilities of characterizing several meaningful modulators of Piezo2 function.

\subsection{Identification of Mtmr2 as a Piezo2 interactor in DRG neurons}

Through the immunoprecipitation based interactomics approach several interactors of Piezo2 were identified by our group (Narayanan et al., 2016). Among the identified proteins, we found three members of a family of phosphatases namely, Mtmr1, Mtmr2 and Mtmr5 (Sbf1). These proteins are members of the myotubularin phosphatase family that are involved in dephosphorylating phosphatidylinositols at the 3-phosphate position (Bolis et al., 2007). Of these, Mtmr2 has been strongly linked to the somatosensory system in lieu of its role in the peripheral neuropathy CMT4B1 (Bolino et al., 2004; Bolis 
et al., 2005; Previtali et al., 2007). Patients with CMT4B1, display severe sensory deficits in the distal limbs with pronounced effect on the sense of vibration (Previtali et al., 2007). Intriguingly, patients with loss of function mutations in Piezo2 also show general losses in vibration detection besides loss in discriminatory touch (Chesler et al., 2016).

The other two phosphatases, Mtmr1 and Mtmr5, identified in our screen were also high probability Piezo2 interactors. Mtmr1, despite being the first identified member of the MTMR family, has not been studied extensively. It displays similar structural features and substrate specificity as Mtmr2 (Bong et al., 2016). And, Mtmr5 is an enzymatically inactive member of the MTMR family and is known to bind to Mtmr2 and increase its enzymatic activity and dictate its subcellular localization (Kim et al., 2003).

Based on available evidence and defined expression in the somatosensory system, I proceeded to characterize Mtmr2 as a potential functional interactor of Piezo2 in DRG neurons. As shown in the results section (section 4.2), I found that loss of Mtmr2 from DRG neurons selectively potentiates Piezo2 mediated RA-MA currents. Concomitantly, overexpression of Mtmr2 in DRG neurons and HEK293 cells suppresses the endogenous or overexpressed Piezo2 RA-MA currents, respectively. Additionally, the catalytic activity of Mtmr2 was found to be essential for its regulation of Piezo2 mediated RA-MA currents. Of note, another member of the MTMR family, Mtmr6 was shown to inhibit $\mathrm{K}_{\mathrm{Ca}} 3.1$ potassium channel activity, through depletion of $\mathrm{PI}(3) \mathrm{P}$ (Srivastava et al., 2005). Therefore it is not entirely unprecedented that Mtmr phosphatases regulate ion channel function.

Remarkably, probing further into the mechanism of Mtmr2-Piezo2 interaction showed that $\mathrm{PI}(3,5) \mathrm{P}_{2}$ may be involved in the interaction. As shown in Figure 8B, Mtmr2 uses $\mathrm{PI}(3) \mathrm{P}$ and $\mathrm{PI}(3,5) \mathrm{P}_{2}$ (the latter preferentially; Berger et al. (2002)) as substrates. An inhibition of Mtmr2 would be expected to increase both $\mathrm{PI}(3,5) \mathrm{P}_{2}$ and $\mathrm{PI}(3) \mathrm{P}$. I used two approaches to counteract this increase of $\mathrm{PI}(3,5) \mathrm{P}_{2}$ upon Mtmr2 knockdown. Apilimod application, to block synthesis of $\mathrm{PI}(3,5) \mathrm{P}_{2}$ (scheme in Figure 27A) and hypotonic intracellular stress to decrease $\mathrm{PI}(3,5) \mathrm{P}_{2}$ levels. Apilimod is a specific blocker of the $\mathrm{PI}(3,5) \mathrm{P}_{2}$ synthesizing enzyme PIKfyve (Vaccari et al., 2011). Its application reverses the Piezo2 potentiation seen upon Mtmr2 knockdown. I also tested the possibility of $\mathrm{PI}(3) \mathrm{P}$ being involved in the modulation of Piezo2 using Wortmannin an inhibitor of the class III PI 3-Kinase which synthesizes PI(3)P (Figure 27A) (Messenger et al., 2015). These experiments showed that Wortmannin application had a much smaller effect on Piezo2 RA-MA currents compared to Apilimod treatment. This could be due to a smaller contribution of $\mathrm{PI}(3) \mathrm{P}$ (compared to $\mathrm{PI}(3,5) \mathrm{P}_{2}$ ) to the Piezo2 potentiation. However, Wortmannin activity is known to be rather unspecific and it is known to also block the class I PI 3-kinase which is involved in the synthesis of $\mathrm{PI}(3,4,5) \mathrm{P}_{3}$ from $\mathrm{PI}(4,5) \mathrm{P}_{2}$ (Messenger et al., 2015). Hence 
(i) Wortmannin could have lower efficiency towards class III PI 3-kinase in our system (Messenger et al., 2015) or (ii) its modulation of the $\mathrm{PI}(4,5) \mathrm{P}_{2}$ pathway may influence the RA-MA currents. It is relevant to note here that activation of TRPV1 suppresses RA-MA currents by depletion of $\mathrm{PI}(4,5) \mathrm{P}_{2}$. Hence, Wortmannin which would be expected to increase $\mathrm{PI}(4,5) \mathrm{P}_{2}$ levels (Messenger et al., 2015) could cause a potentiation of Piezo2 through this alternative pathway thereby confounding the study of $\mathrm{PI}(3) \mathrm{P}$ effect on Piezo2 RA-MA currents. Though these data prove inconclusive in elucidating the role for $\mathrm{PI}(3) \mathrm{P}$ in the regulation of Piezo2, they provide interesting hints towards cross-talk between membrane lipids in regulation of Piezo2. Notwithstanding, the Apilimod data strongly indicate a role for $\mathrm{PI}(3,5) \mathrm{P}_{2}$ in the interaction between Piezo2 and Mtmr2. In order to test this using another method, I exploited the fact the $\mathrm{PI}(3,5) \mathrm{P}_{2}$ levels are transiently but robustly modulated in response to osmotic stress (Previtali et al., 2007). Osmotic stress applied intracellularly to decrease levels of $\mathrm{PI}(3,5) \mathrm{P}_{2}$ was able to counteract the Piezo2 RA-MA potentiation caused by the loss of Mtmr2. Both these approaches indicate that $\mathrm{PI}(3,5) \mathrm{P}_{2}$ (and to a yet unknown extent $\mathrm{PI}(3) \mathrm{P}$ ) may play a role in the Piezo2 and Mtmr2 interaction.

Additional evidence that Piezo2, Mtmr2 and $\mathrm{PI}(3,5) \mathrm{P}_{2}$ interact on a cellular level came from biochemical binding assays. I found evidence to indicate that Piezo2 can bind directly to $\mathrm{PI}(3,5) \mathrm{P}_{2}$. I identified a poly-basic intracellular peptide sequence in the Piezo2 structure which could bind $\mathrm{PI}(3,5) \mathrm{P}_{2}$ in vitro. Interestingly, the same Piezo2 fragment was also able to bind an Mtmr2 $\mathrm{Pl}(3,5) \mathrm{P}_{2}$-binding peptide. The Piezo2-Mtmr2 binding appeared to be independent of the presence or absence of $\mathrm{PI}(3,5) \mathrm{P}_{2}$. Further, the binding of the Piezo2 peptide to $\mathrm{PI}(3,5) \mathrm{P}_{2}$ and $\mathrm{Mtmr}$ peptide was dependent on the basic amino acids in the sequence as shown by the inability of the mutant $3 Q$ peptide to bind $\mathrm{PI}(3,5) \mathrm{P}_{2}$. The data hint towards a potential tripartite interaction between Piezo2, Mtmr2 and $\mathrm{PI}(3,5) \mathrm{P}_{2}$ mediated by charged amino acids.

The next question was if $\mathrm{PI}(3,5) \mathrm{P}_{2}$ can independently regulate Piezo2. Towards this goal Apilimod was applied to untreated DRG neurons and RA-MA currents measured. Curiously RA-MA currents remained unaffected, which could be explained by the low baseline expression of $\mathrm{PI}(3,5) \mathrm{P}_{2}$ in cells. Therefore under physiological conditions, decreasing $\mathrm{PI}(3,5) \mathrm{P}_{2}$ may not have a cellular consequence. Additionally I also tried to artificially increase the levels of cellular $\mathrm{PI}(3,5) \mathrm{P}_{2}$ by addition of synthetic diC8 $\mathrm{PI}(3,5) \mathrm{P}_{2}$ to DRG neurons. No effect on RA-MA currents was observed even at saturating concentrations of $\mathrm{PI}(3,5) \mathrm{P}_{2}$. This could be explained either by mis-localization of synthetics PIPs in the cells or because of a fast turnover of $\mathrm{PI}(3,5) \mathrm{P}_{2}$ through the action of cellular phosphatases. 
However, paradigms involving application of osmotic stress to DRG neurons provides evidence for a direct regulation of Piezo2 mediated currents and behavior by $\mathrm{PI}(3,5) \mathrm{P}_{2}$. As described earlier, subjecting cells to osmotic stress regulates levels of $\mathrm{PI}(3,5) \mathrm{P}_{2}$ transiently and drastically. Additionally, osmotic stress was also shown to potentiate Piezo2 mediated currents and behaviors (as seen from our own data Figure $\mathbf{3 1}$ and previously published data from Jia and colleagues (Jia et al., 2016)). Interestingly, upon application of PIKfyve blocker Apilimod, to neurons subjected to osmotic stress, the potentiation of Piezo2 currents can be reversed. Furthermore osmotic stress induced mechanical allodynia in mouse paw can be reversed by application of Apilimod. These data point to a direct regulation of the Piezo2 channel by $\mathrm{PI}(3,5) \mathrm{P}_{2}$.

There are several possibilities of how $\mathrm{PI}(3,5) \mathrm{P}_{2}$ and Mtmr2 may regulate Piezo2. Mtmr2 may bind Piezo2 to facilitate its enrichment in $\mathrm{PI}(3,5) \mathrm{P}_{2}$ rich micro domains within the plasma membrane. A schematic of this hypothesis is illustrated in Figure 33 . In the proposed mechanism Mtmr2 binds to Piezo2 at the plasma membrane and under normal conditions, limits the amount of $\mathrm{PI}(3,5) \mathrm{P}_{2}$ by catalyzing its rapid turnover. Loss of Mtmr2 (through Mtmr2 siRNA) causes an increase in the local $\mathrm{PI}(3,5) \mathrm{P}_{2}$. This increased $\mathrm{PI}(3,5) \mathrm{P}_{2}$ potentiates Piezo2 currents through a yet unknown mechanism. On the other hand, an increase in cellular Mtmr2 (through Mtrm2 overexpression) causes increased turnover of $\mathrm{PI}(3,5) \mathrm{P}_{2}$ levels decreasing it below baseline. This causes a suppression of the Piezo2 activity.

A highly speculative scenario for this potentiation is that the electrostatic binding of Piezo2 to the excessive $\mathrm{PI}(3,5) \mathrm{P}_{2}$ causes structural changes in the channel causing an increase in the channel activity. A similar regulation is seen with TRPML1 where it was proposed that $\mathrm{PI}(3,5) \mathrm{P}_{2}$ sensitizes TRPML1 making it easier to activate (Dong et al., 2010; Shen et al., 2011). A comparable process may operate during $\mathrm{PI}(3,5) \mathrm{P}_{2}$ regulation of Piezo2, though in order to study this, a detailed understanding of the Piezo2 structure and the mechanism of its conduction would be required. Another possibility is that $\mathrm{PI}(3,5) \mathrm{P}_{2}$ acts as a secondary messenger and initiates downstream signaling cascades which influence Piezo2 function. The $\mathrm{PI}(4,5) \mathrm{P}_{2}$ mediated regulation of Piezo2 upon TRPV1 activation relies on such a pathway (Borbiro et al., 2015).

In order to study the Piezo2-Mtmr2-PI(3,5) $\mathrm{P}_{2}$ interaction further, we need tools to faithfully visualize the proteins in the native system and how they may re-distribute/re-arrange upon application of stimuli. Though there are fluorescent probes that can detect membrane lipids in native cells, none of them have been shown to be useful for the detection of $\mathrm{PI}(3,5) \mathrm{P}_{2}$ (Hammond et al., 2015; Li et al., 2013). The extremely low cellular abundance of $\mathrm{PI}(3,5) \mathrm{P}_{2}$ poses a challenge to explore its role under physiological conditions. Furthermore, there are two Mtmr2 knockout mice used commonly in the field 
as models for CMT4B1 (Bolino et al., 2004; Bonneick et al., 2005). We unfortunately did not have access to these mice for this study, but a somatosensory characterization of these mice could provide interesting hints towards the role of Mtmr2 in Piezo2 mediated behaviors. Also, whether Mtmr2-Piezo2 interaction has a role to play in the peripheral neuropathy (CMT4B1) could be answered through experiments with these mice.

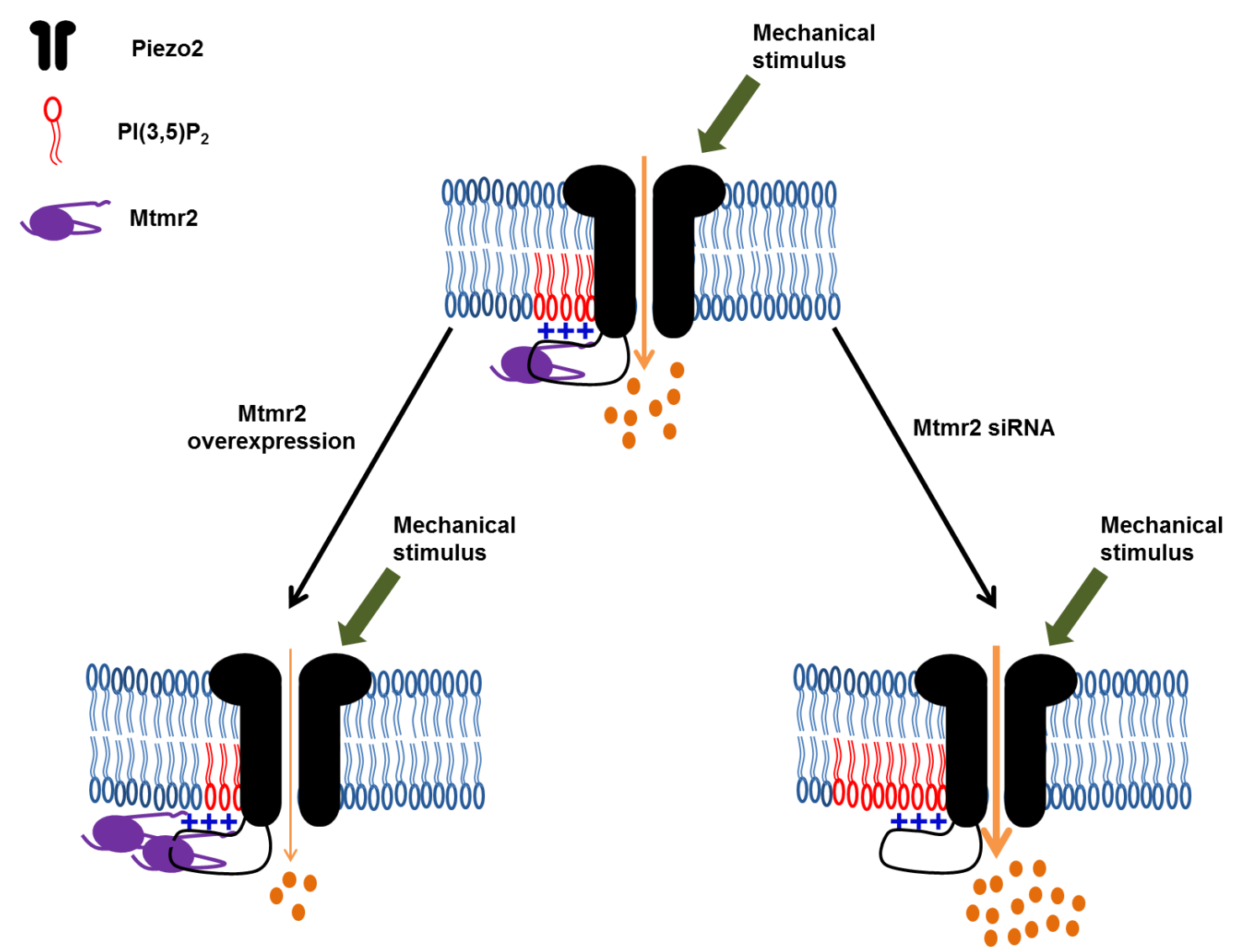

Figure 33: Illustration of Piezo2-PI(3,5) $\mathrm{P}_{2}-\mathrm{Mtmr2}$ interaction

Under normal conditions Mtmr2 binds to Piezo2 and maintains it close to the local $\mathrm{PI}(3,5) \mathrm{P}_{2}$ membrane pools. Under these conditions, Mtmr2 maintains the amount of $\mathrm{PI}(3,5) \mathrm{P}_{2}$ by regulating its turnover. Loss of Mtmr2 (through Mtmr2 siRNA) leads to an increase in the local levels of $\mathrm{PI}(3,5) \mathrm{P}_{2}$ leading to a potentiation of Piezo2 (possibly through structural changes in Piezo2). Further, overexpression of Mtmr2 in the cells causes increased turnover of $\mathrm{PI}(3,5) \mathrm{P}_{2}$, decreasing it cellular availability. This leads to a suppression of the Piezo2 mediated RA-MA currents. How $\mathrm{PI}(3,5) \mathrm{P}_{2}$ availability regulates the Piezo2 function remains to be seen, it could be speculated that either structural changes in Piezo2 or action of secondary messenger mediated signaling cascades could play a role. 


\subsection{Piezo2 function is controlled by a defined set of interacting proteins}

In this study I characterized two interactors of Piezo2 in somatosensory neurons. Both proteins were identified through a mass-spectrometry based interactomics approach. The immunoprecipitation was done using the commercial Piezo2 antibody (NBP1-78624; Narayanan et al. (2016)) which was tested to reliably detect Piezo2 in mass-spectrometry experiments (Narayanan et al., 2016).

In 2014, Woo and colleagues reported the generation of an anti-Piezo2 antibody, which showed specificity on tissue from Piezo2 ${ }^{G F P}$ and skin-specific Piezo2 knockout mice (Woo et al., 2014). Using this antibody, we performed an additional immunoprecipitation based mass-spectrometry screen (Performed by Dr. Tom Rouwette and Julia Sondermann, Somatosensory Signaling and Systems Biology group). Surprisingly we found a distinct set of interactors using this antibody. (Of note, this experiment was only repeated twice and complete statistics could not be performed). Yet the difference in the list of proteins identified is surprising. There may be various explanations for the differences we observed. First, the two antibodies differ in the region of Piezo2 they bind (Antigenic peptide for NBP1-78624; Novus Biologicals) corresponds to the 1600-1650 amino acids region, close to C-term of Piezo2; Antigenic peptide for anti-Piezo2 antibody from Woo and colleagues corresponds to 351-485 amino acid peptide, close to the $\mathrm{N}$-term of the protein). This could affect the secondary structure of the protein and thereby influence the binding properties. Additionally, depending on the strength of anti-Piezo2 antibody binding to Piezo2, it could also influence the binding properties of the interacting partners. Therefore, identification of weak or transient interactors may vary depending on the strength of the anti-Piezo2 antibody.

Due to the significant differences in the protein lists, I tested if the proteins identified on the new screen also constituted bona fide Piezo2 interactors. For this purpose, two proteins namely; MAP7 domain containing protein (MAP7d2) and Lunapark (Lnp) were chosen. MAP7d2 was identified as a X-linked maternal imprinted gene in humans which is expressed highly in the brain (Niida and Yachie, 2011). Though there have not been many studies on MAP7d2, recently Tymanskyj and colleagues showed the MAP7 regulates the development of axon collateral branches in DRG neurons. These axon collaterals are essential for DRG neurons to form multiple synaptic connections (Tymanskyj et al., 2017). Lunapark was identified in 2003 by Spitz and colleagues in the developing digits with similar profile as development related Hox genes (Spitz et al., 2003). It was later shown to be required for ER network formation. Structurally, Lnp contains two transmembrane domains and a zinc finger motif (Chen et al., 2013). Both proteins are newly discovered and there is not much information on their cellular function and no roles have been described in the context of somatosensation. As a preliminary 
analysis, I tested if suppression of these proteins in DRG neurons would impact Piezo2 mediated RA-MA currents. Surprisingly I did not observe any impact on the Piezo2 mediated currents upon loss of either of the proteins (data not shown). This could be because the tested proteins may simply be part of a complex modulating Piezo2 and there may be other cellular components with compensatory functions. The lack of functional interaction could also mean that the new anti-Piezo2 antibody could not robustly identify Piezo2 interactors. Nevertheless, these experiments do demonstrate that not all proteins expressed in DRG neurons can influence Piezo2 function. Combining this with the functional data reporting interaction of Piezo2 with Pcnt and Mtmr2, it underlines the fact that there is indeed a defined complex of proteins that regulate Piezo2 function in vitro. The lack of consensus between the two mass spectrometry screens also highlights the need to study the Piezo2 interactors through multiple approaches. Complementary methods to identify interactors of Piezo2 could include bioinformatics approaches based on genomic information or protein structures, genomic approaches like yeast two-hybrid screening and biochemical approaches using cross-linking of associated proteins prior to co-immunoprecipitation (Ivanov et al., 2011). Further orthogonal validation of the interactions is essential to establish proteins as bona fide interactors of Piezo2 in vitro and in vivo. 


\section{REFERENCES}

Abraira, V.E., and Ginty, D.D. (2013). The sensory neurons of touch. Neuron 79, 618-639.

Alcaino, C., Knutson, K., Gottlieb, P.A., Farrugia, G., and Beyder, A. (2017). Mechanosensitive ion channel Piezo2 is inhibited by D-GsMTx4. Channels 11, 1-9.

Arnadóttir, J., and Chalfie, M. (2010). Eukaryotic mechanosensitive channels. Annual review of biophysics 39, 111-137.

Avenali, L., Narayanan, P., Rouwette, T., Cervellini, I., Sereda, M., Gomez-Varela, D., and Schmidt, M. (2014). Annexin A2 Regulates TRPA1-Dependent Nociception. Journal of Neuroscience 34, 14506-14516.

Bagriantsev, S.N., Gracheva, E.O., and Gallagher, P.G. (2014). Piezo proteins: Regulators of mechanosensation and other cellular processes. Journal of Biological Chemistry 289.

Begley, M.J., Taylor, G.S., Brock, M.A., Ghosh, P., Woods, V.L., and Dixon, J.E. (2006). Molecular basis for substrate recognition by MTMR2, a myotubularin family phosphoinositide phosphatase. Proc Natl Acad Sci U S A 103, 927-932.

Berger, P., Bonneick, S., Willi, S., Wymann, M., and Suter, U. (2002). Loss of phosphatase activity in myotubularin-related protein 2 is associated with Charcot-Marie-Tooth disease type 4B1. Human molecular genetics 11, 1569-1579.

Berger, P., Schaffitzel, C., Berger, I., Ban, N., and Suter, U. (2003). Membrane association of myotubularin-related protein 2 is mediated by a pleckstrin homology-GRAM domain and a coiled-coil dimerization module. Proceedings of the National Academy of Sciences of the United States of America 100, 12177-12182.

Bolino, A., Bolis, A., Previtali, S.C., Dina, G., Bussini, S., Dati, G., Amadio, S., Del Carro, U., Mruk, D.D., Feltri, M.L., et al. (2004). Disruption of Mtmr2 CMT4B1-like neuropathy with myelin outfolding and impaired spermatogenesis. Journal of Cell Biology 167, 711-721.

Bolino, A., Marigo, V., Ferrera, F., Loader, J., Romio, L., Leoni, A., Di Duca, M., Cinti, R., Cecchi, C., Feltri, M.L., et al. (2002). Molecular characterization and expression analysis of Mtmr2, mouse homologue of MTMR2, the myotubularin-related 2 gene, mutated in CMT4B. Gene 283, 17-26.

Bolis, A., Coviello, S., Bussini, S., Dina, G., Pardini, C., Previtali, S.C., Malaguti, M., Morana, P., Del Carro, U., Feltri, M.L., et al. (2005). Loss of Mtmr2 phosphatase in Schwann cells but not in motor neurons causes Charcot-Marie-Tooth type 4B1 neuropathy with myelin outfoldings. The Journal of neuroscience : the official journal of the Society for Neuroscience 25, 8567-8577.

Bolis, A., Coviello, S., Visigalli, I., Taveggia, C., Bachi, A., Chishti, A.H., Hanada, T., Quattrini, A., Previtali, S.C., Biffi, A., et al. (2009). Dlg1, Sec8, and Mtmr2 regulate membrane homeostasis in Schwann cell myelination. The Journal of neuroscience : the official journal of the Society for Neuroscience 29, 8858-8870. 
Bolis, A., Zordan, P., Coviello, S., and Bolino, A. (2007). Myotubularin-related (MTMR) phospholipid phosphatase proteins in the peripheral nervous system. Molecular Neurobiology 35, 308-316.

Bong, S.M., Son, K.-B., Yang, S.-W., Park, J.-W., Cho, J.-W., Kim, K.-T., Kim, H., Kim, S.J., Kim, Y.J., and Lee, B.I. (2016). Crystal Structure of Human Myotubularin-Related Protein 1 Provides Insight into the Structural Basis of Substrate Specificity. PloS one 11, e0152611.

Bonneick, S., Boentert, M., Berger, P., Atanasoski, S., Mantei, N., Wessig, C., Toyka, K.V., Young, P., and Suter, U. (2005). An animal model for Charcot-Marie-Tooth disease type 4B1. Human Molecular Genetics 14, 3685-3695.

Borbiro, I., Badheka, D., and Rohacs, T. (2015). Activation of TRPV1 channels inhibits mechanosensitive Piezo channel activity by depleting membrane phosphoinositides. Science Signalling 8, 1-12.

Brehm, P., Kullberg, R., and Moody-Corbett, F. (1984). Properties of Non-Junctional Acetylcholine Receptor Channels On Innervated Muscle Of Xenopus Laevis. The Journal of Physiology 350, 631-648.

Buchman, J.J., Tseng, H.C., Zhou, Y., Frank, C.L., Xie, Z., and Tsai, L.H. (2010). Cdk5rap2 interacts with pericentrin to maintain the neural progenitor pool in the developing neocortex. Neuron 66, 386-402.

Cai, X., Xu, Y., Cheung, A.K., Tomlinson, R.C., Alc??zar-Rom??n, A., Murphy, L., Billich, A., Zhang, B., Feng, Y., Klumpp, M., et al. (2013). PIKfyve, a class III PI Kinase, is the target of the small molecular IL-12/IL-23 inhibitor apilimod and a player in toll-like receptor signaling. Chemistry and Biology 20, 912-921.

Chalfie, M. (2009). Neurosensory mechanotransduction. Nature Reviews Molecular Cell Biology 10, 44-52.

Chen, C.-T., Hehnly, H., Yu, Q., Farkas, D., Zheng, G., Redick, Sambra D., Hung, H.-F., Samtani, R., Jurczyk, A., Akbarian, S., et al. (2014). A Unique Set of Centrosome Proteins Requires Pericentrin for Spindle-Pole Localization and Spindle Orientation. Current Biology 24, 2327-2334.

Chen, D., Purohit, A., Halilovic, E., Doxsey, S.J., and Newton, A.C. (2004). Centrosomal Anchoring of Protein Kinase C $\beta$ II by Pericentrin Controls Microtubule Organization, Spindle Function, and Cytokinesis. Journal of Biological Chemistry 279, 4829-4839.

Chen, S., Novick, P., and Ferro-Novick, S. (2013). ER network formation requires a balance of the dynamin-like GTPase Sey1p and Lnp1p, a member of the Lunapark family. Nature Cell Biology 14, 707-716.

Chesler, A.T., Szczot, M., Bharucha-Goebel, D., Čeko, M., Donkervoort, S., Laubacher, C., Hayes, L.H., Alter, K., Zampieri, C., Stanley, C., et al. (2016). The Role of $<i>P I E Z O 2</ i>$ in Human Mechanosensation. New England Journal of Medicine 375, 1355-1364. 
Clague, M.J., and Lorenzo, Ó. (2005). The myotubularin family of lipid phosphatases. Traffic 6, 1063-1069.

Coste, B., Houge, G., Murray, M.F., Stitziel, N., Bandell, M., Giovanni, M.a., Philippakis, A., Hoischen, A., Riemer, G., Steen, U., et al. (2013). Gain-of-function mutations in the mechanically activated ion channel PIEZO2 cause a subtype of Distal Arthrogryposis. Proceedings of the National Academy of Sciences of the United States of America 110, 4667-4672.

Coste, B., Mathur, J., Schmidt, M., Earley, T.J., Ranade, S., Petrus, M.J., Dubin, A.E., and Patapoutian, A. (2010). Piezo1 and Piezo2 are essential components of distinct mechanically-activated cation channels. Science 330, 55-60.

Coste, B., Murthy, S.E., Mathur, J., Schmidt, M., Mechioukhi, Y., Delmas, P., and Patapoutian, A. (2015). Piezo1 ion channel pore properties are dictated by C-terminal region. Nature communications 6, 7223.

Coste, B., Xiao, B., Santos, J.S., Syeda, R., Grandl, J., Spencer, K.S., Kim, S.E., Schmidt, M., Mathur, J., Dubin, A.E., et al. (2012). Piezo proteins are pore-forming subunits of mechanically activated channels. Nature 483, 176-181.

Dammermann, a. (2002). Assembly of centrosomal proteins and microtubule organization depends on PCM-1. The Journal of cell biology 159, 255-266.

Delaval, B., and Doxsey, S.J. (2010). Pericentrin in cellular function and disease. The Journal of cell biology 188, 181-190.

Delmas, P., and Coste, B. (2013). Mechano-gated ion channels in sensory systems. Cell 155, 278-284.

Delmas, P., Hao, J., and Rodat-Despoix, L. (2011). Molecular mechanisms of mechanotransduction in mammalian sensory neurons. Nature reviews Neuroscience 12, 139-153.

Dong, X.-p., Shen, D., Wang, X., Dawson, T., Li, X., Zhang, Q., Cheng, X., Zhang, Y., Weisman, L.S., Delling, M., et al. (2010). PI(3,5)P2 Mucolipin Ca2+ Controls Membrane Traffic by Direct Activation of Release Channels in the Endolysosome. Nature 1, 1-21.

Doxsey, S.J., Stein, P., Evans, L., Calarco, P.D., and Kirschner, M. (1994). Pericentrin, a highly conserved centrosome protein involved in microtubule organization. Cell 76, 639-650.

Drew, L.J., and Wood, J.N. (2007). FM1-43 is a permeant blocker of mechanosensitive ion channels in sensory neurons and inhibits behavioural responses to mechanical stimuli. Molecular pain 3,1.

Dubin, A.E., Schmidt, M., Mathur, J., Petrus, M.J., Xiao, B., and Coste, B. (2012). Inflammatory Signals Enhance Piezo2-Mediated Mechanosensitive Currents. CellReports 2, 511-517. 
Eijkelkamp, N., Linley, J.E., Torres, J.M., Bee, L., Dickenson, a.H., Gringhuis, M., Minett, M.S., Hong, G.S., Lee, E., Oh, U., et al. (2013). A role for Piezo2 in EPAC1-dependent mechanical allodynia. Nature communications 4, 1682.

Endoh-Yamagami, S., Karkar, K.M., May, S.R., Cobos, I., Thwin, M.T., Long, J.E., Ashique, A.M., Zarbalis, K., Rubenstein, J.L.R., and Peterson, A.S. (2010). A mutation in the pericentrin gene causes abnormal interneuron migration to the olfactory bulb in mice. Developmental Biology 340, 41-53.

Ernstrom, G.G., and Chalfie, M. (2002). Genetics of sensory mechanotransduction. Annual review of genetics 36, 411-453.

Ferrari, L.F., Bogen, O., Green, P., and Levine, J.D. (2015). Contribution of Piezo2 to endothelium-dependent pain. Molecular Pain 11, 65.

Florez-Paz, D., Bali, K.K., Kuner, R., and Gomis, A. (2016). A critical role for Piezo2 channels in the mechanotransduction of mouse proprioceptive neurons. Scientific Reports 6 , 25923.

Gamper, N., and Shapiro, M.S. (2007). Regulation of ion transport proteins by membrane phosphoinositides. Nat Rev Neurosci 8, 921-934.

Ge, J., Li, W., Zhao, Q., Li, N., Chen, M., Zhi, P., Li, R., Gao, N., Xiao, B., and Yang, M. (2015). Architecture of the mammalian mechanosensitive Piezo1 channel. Nature 527.

Gillespie, P.G., and Walker, R.G. (2001). Molecular basis of mechanosensory transduction. Nature 413, 194-202.

Griffith, E., Walker, S., Martin, C.-A., Vagnarelli, P., Stiff, T., Vernay, B., Al Sanna, N., Saggar, A., Hamel, B., Earnshaw, W.C., et al. (2008). Mutations in pericentrin cause Seckel syndrome with defective ATR-dependent DNA damage signaling. Nature genetics 40, $232-$ 236.

Guharay, F., and Sachs, F. (1984). Stretch-activated single ion channel currents in tissuecultured embryonic chick skeletal muscle. The Journal of physiology 352, 685-701.

Hammond, G.R.V., Takasuga, S., Sasaki, T., and Balla, T. (2015). The ML1Nx2 phosphatidylinositol 3,5-bisphosphate probe shows poor selectivity in cells. PLoS ONE 10,113.

Hanack, C., Moroni, M., Lima, W.C., Wende, H., Kirchner, M., Adelfinger, L., SchrenkSiemens, K., Tappe-Theodor, A., Wetzel, C., Kuich, P.H., et al. (2015). GABA blocks pathological but not acute TRPV1 pain signals. Cell 160, 759-770.

Hao, J., and Delmas, P. (2011). Recording of mechanosensitive currents using piezoelectrically driven mechanostimulator. Nature protocols 6, 979-990.

Hao, J., Padilla, F., Dandonneau, M., Lavebratt, C., Lesage, F., Noël, J., and Delmas, P. (2013). Kv1.1 channels act as mechanical brake in the senses of touch and pain. Neuron 77 , 899-914. 
Hong, G.-s., Lee, B., Wee, J., Chun, H., Kim, H., Jung, J., Cha, J.Y., Riew, T.-R., Kim, G.H., Kim, I.-B., et al. (2016). Tentonin 3/TMEM150c Confers Distinct Mechanosensitive Currents in Dorsal-Root Ganglion Neurons with Proprioceptive Function. Neuron 91, 107-118.

Hu, J., Chiang, L.-Y., Koch, M., and Lewin, G.R. (2010). Evidence for a protein tether involved in somatic touch. The EMBO journal 29, 855-867.

Huang da, W., Sherman, B.T., and Lempicki, R.A. (2009). Systematic and integrative analysis of large gene lists using DAVID bioinformatics resources. Nature Protocols 4, 44-57. Huang, D.W., Sherman, B.T., and Lempicki, R.A. (2009). Bioinformatics enrichment tools: Paths toward the comprehensive functional analysis of large gene lists. Nucleic Acids Research 37, 1-13.

Hudmon, A., and Schulman, H. (2002). Neuronal CA2+/calmodulin-dependent protein kinase II: the role of structure and autoregulation in cellular function. Annual Review of Biochemistry 71, 473-510.

Ikeda, R., Cha, M., Ling, J., Jia, Z., Coyle, D., and Gu, J.G. (2014). Merkel cells transduce and encode tactile stimuli to drive Aß-afferent impulses. Cell 157, 664-675.

Ivanov, a.S., Zgoda, V.G., and Archakov, a.I. (2011). Technologies of protein interactomics: A review. Russian Journal of Bioorganic Chemistry 37, 4-16.

Jia, Z., Ikeda, R., Ling, J., Viatchenko-Karpinski, V., and Gu, J.G. (2016). Regulation of Piezo2 mechanotransduction by static plasma membrane tension in primary afferent neurons. Journal of Biological Chemistry, jbc.M115.692384.

Jin, N., Lang, M.J., and Weisman, L.S. (2016). Phosphatidylinositol 3,5-bisphosphate: regulation of cellular events in space and time. Biochemical Society Transactions 44, 177184.

Julius, D., and Basbaum, a.I. (2001). Molecular mechanisms of nociception. Nature 413, 203-210.

Jurczyk, A., Gromley, A., Redick, S., San Agustin, J., Witman, G., Pazour, G.J., Peters, D.J., and Doxsey, S. (2004). Pericentrin forms a complex with intraflagellar transport proteins and polycystin-2 and is required for primary cilia assembly. Journal of Cell Biology 166, 637-643. Jurczyk, A., Pino, S.C., O'Sullivan-Murphy, B., Addorio, M., Lidstone, E.a., Diiorio, P., Lipson, K.L., Standley, C., Fogarty, K., Lifshitz, L., et al. (2010). A novel role for the centrosomal protein, pericentrin, in regulation of insulin secretory vesicle docking in mouse pancreatic beta-cells. PloS one 5, e11812.

Kaidoh, T., and Inoué, T. (2000). Intercellular junctions between palisade nerve endings and outer root sheath cells of rat vellus hairs. The Journal of comparative neurology 420, 419427. 
Kamajaya, A., Kaiser, Jens T., Lee, J., Reid, M., and Rees, Douglas C. (2014). The Structure of a Conserved Piezo Channel Domain Reveals a Topologically Distinct $\beta$ Sandwich Fold. Structure 22, 1520-1527.

Kandel, E.R., Schwartz, J.H., and Jessell, T.M. (2000). Principles of Neural Science.

Kim, S.-A., Vacratsis, P.O., Firestein, R., Cleary, M.L., and Dixon, J.E. (2003). Regulation of myotubularin-related (MTMR)2 phosphatidylinositol phosphatase by MTMR5, a catalytically inactive phosphatase. Proceedings of the National Academy of Sciences of the United States of America 100, 4492-4497.

Laporte, J., Blondeau, F., Buj-Bello, A., Tentler, D., Kretz, C., Dahl, N., and Mandel, J.L. (1998). Characterization of the myotubularin dual specificity phosphatase gene family from yeast to human. Human Molecular Genetics 7, 1703-1712.

Lechner, S.G., Markworth, S.r., Poole, K., Smith, E.S.J., Lapatsina, L., Frahm, S., May, M., Pischke, S., Suzuki, M., Iba??ez-Tallon, I.s., et al. (2011). The molecular and cellular identity of peripheral osmoreceptors. Neuron 69, 332-344.

Lee, H.W., Kim, Y., Han, K., Kim, H., and Kim, E. (2010). The phosphoinositide 3phosphatase MTMR2 interacts with PSD-95 and maintains excitatory synapses by modulating endosomal traffic. The Journal of neuroscience : the official journal of the Society for Neuroscience 30, 5508-5518.

Levitan, I.B. (2006). Signaling protein complexes associated with neuronal ion channels. Nature Neuroscience 9, 305-310.

Li, L., and Ginty, D.D. (2014). The structure and organization of lanceolate mechanosensory complexes at mouse hair follicles. eLife 2014, 1-24.

Li, L., Rutlin, M., Abraira, Victoria E., Cassidy, C., Kus, L., Gong, S., Jankowski, Michael P., Luo, W., Heintz, N., Koerber, H.R., et al. (2011). The Functional Organization of Cutaneous Low-Threshold Mechanosensory Neurons. Cell 147, 1615-1627.

Li, Q., Hansen, D., Killilea, a., Joshi, H.C., Palazzo, R.E., and Balczon, R. (2001). Kendrin/pericentrin-B, a centrosome protein with homology to pericentrin that complexes with PCM-1. Journal of cell science 114, 797-809.

Li, X., Wang, X., Zhang, X., Zhao, M., Tsang, W.L., Zhang, Y., Yau, R.G.W., Weisman, L.S., and $\mathrm{Xu}, \mathrm{H}$. (2013). Genetically encoded fluorescent probe to visualize intracellular phosphatidylinositol 3,5-bisphosphate localization and dynamics. Proceedings of the National Academy of Sciences of the United States of America 110, 21165-21170.

Liu, L., Chen, L., Liedtke, W., and Simon, S.a. (2007). Changes in osmolality sensitize the response to capsaicin in trigeminal sensory neurons. Journal of neurophysiology 97,2001 2015.

Lolignier, S., Eijkelkamp, N., and Wood, J.N. (2014). Mechanical allodynia. Pflugers Archiv : European journal of physiology, 133-139. 
Lorenzo, O., Urbé, S., and Clague, M.J. (2006). Systematic analysis of myotubularins: heteromeric interactions, subcellular localisation and endosome related functions. Journal of cell science 119, 2953-2959.

Lou, S., Duan, B., Vong, L., Lowell, B.B., and Ma, Q. (2013). Runx1 controls terminal morphology and mechanosensitivity of VGLUT3-expressing C-mechanoreceptors. The Journal of neuroscience : the official journal of the Society for Neuroscience 33, 870-882.

Maksimovic, S., Nakatani, M., Baba, Y., Nelson, A.M., Marshall, K.L., Wellnitz, S.a., Firozi, P., Woo, S.-H., Ranade, S., Patapoutian, A., et al. (2014). Epidermal Merkel cells are mechanosensory cells that tune mammalian touch receptors. Nature 509,617-621.

Martinez-Campos, M., Basto, R., Baker, J., Kernan, M., and Raff, J.W. (2004). The Drosophila pericentrin-like protein is essential for cilia/flagella function, but appears to be dispensable for mitosis. The Journal of cell biology 165, 673-683.

McCartney, A.J., Zhang, Y., and Weisman, L.S. (2014a). Phosphatidylinositol 3,5bisphosphate: low abundance, high significance. Bioessays 36, 52-64.

McCartney, A.J., Zolov, S.N., Kauffman, E.J., Zhang, Y., Strunk, B.S., Weisman, L.S., and Sutton, M.A. (2014b). Activity-dependent PI(3,5)P2 synthesis controls AMPA receptor trafficking during synaptic depression. Proceedings of the National Academy of Sciences of the United States of America 111, E4896-4905.

McMillin, M.J., Beck, A.E., Chong, J.X., Shively, K.M., Buckingham, K.J., Gildersleeve, H.I.S., Aracena, M.I., Aylsworth, A.S., Bitoun, P., Carey, J.C., et al. (2014). Mutations in PIEZO2 cause Gordon syndrome, Marden-Walker Syndrome, and distal arthrogryposis type 5. American Journal of Human Genetics 94.

Messenger, S.W., Thomas, D.D.H., Cooley, M.M., Jones, E.K., Falkowski, M.A., August, B.K., Fernandez, L.A., Gorelick, F.S., and Groblewski, G.E. (2015). Early to Late Endosome Trafficking Controls Secretion and Zymogen Activation in Rodent and Human Pancreatic Acinar Cells. Cmgh 1, 695-709.

Mironova, Y.A., Lenk, G.M., Lin, J.P., Lee, S.J., Twiss, J.L., Vaccari, I., Bolino, A., Havton, L.A., Min, S.H., Abrams, C.S., et al. (2016). PI(3,5)P2 biosynthesis regulates oligodendrocyte differentiation by intrinsic and extrinsic mechanisms. eLife 5, 1-29.

Miyoshi, K., Asanuma, M., Miyazaki, I., Matsuzaki, S., Tohyama, M., and Ogawa, N. (2006). Characterization of pericentrin isoforms in vivo. Biochemical and biophysical research communications 351, 745-749.

Miyoshi, K., Kasahara, K., Miyazaki, I., Shimizu, S., Taniguchi, M., Matsuzaki, S., Tohyama, M., and Asanuma, M. (2009). Pericentrin, a centrosomal protein related to microcephalic primordial dwarfism, is required for olfactory cilia assembly in mice. FASEB journal : official publication of the Federation of American Societies for Experimental Biology 23, 3289-3297. 
Mo, G., Bernier, L.-P., Zhao, Q., Chabot-Doré, A.-J., Ase, A.R., Logothetis, D., Cao, C.-Q., and Séguéla, P. (2009). Subtype-Specific Regulation of P2X3 and P2X2/3 Receptors by Phosphoinositides in Peripheral Nociceptors. Molecular Pain 5, 1744-8069-1745-1747.

Mühlhans, J., Brandstätter, J.H., and Giessl, A. (2011). The centrosomal protein pericentrin identified at the basal body complex of the connecting cilium in mouse photoreceptors. PloS one 6, e26496.

Mühlhans, J., and GießI, A. (2012). Pericentrin in health and disease: Exploring the patchwork of pericentrin splice variants. Communicative and Integrative Biology 5, 304-307.

Narayanan, P., Sondermann, J., Rouwette, T., Karaca, S., Urlaub, H., Mitkovski, M., GomezVarela, D., and Schmidt, M. (2016). Native Piezo2 interactomics identifies pericentrin as a novel regulator of Piezo2 in somatosensory neurons. Journal of Proteome Research 15, 2676-2687.

Nauli, S.M., Alenghat, F.J., Luo, Y., Williams, E., Vassilev, P., Li, X., Elia, A.E.H., Lu, W., Brown, E.M., Quinn, S.J., et al. (2003). Polycystins 1 and 2 mediate mechanosensation in the primary cilium of kidney cells. Nature genetics 33, 129-137.

Ng, A.A., Logan, A.M., Schmidt, E.J., and Robinson, F.L. (2013). The CMT4B diseasecausing phosphatases Mtmr2 and Mtmr13 localize to the Schwann cell cytoplasm and endomembrane compartments, where they depend upon each other to achieve wild-type levels of protein expression. Human Molecular Genetics 22, 1493-1506.

Niida, Y., and Yachie, A. (2011). MAP7D2 is a brain expressing X-linked maternal imprinted gene in humans. Nature Preceedings: Pre-publication Research and Preliminary Findings.

Poole, K., Herget, R., Lapatsina, L., Ngo, H.-D., and Lewin, G.R. (2014). Tuning Piezo ion channels to detect molecular-scale movements relevant for fine touch. Nature communications 5, 3520 .

Previtali, S.C., Quattrini, A., and Bolino, A. (2007). Charcot-Marie-Tooth type 4B demyelinating neuropathy: deciphering the role of MTMR phosphatases. Expert reviews in molecular medicine 9, 1-16.

Previtali, S.C., Zerega, B., Sherman, D.L., Brophy, P.J., Dina, G., King, R.H.M., Salih, M.M., Feltri, L., Quattrini, A., Ravazzolo, R., et al. (2003). Myotubularin-related 2 protein phosphatase and neurofilament light chain protein, both mutated in CMT neuropathies, interact in peripheral nerve. Human Molecular Genetics 12, 1713-1723.

Purohit, A., Tynan, S.H., Valle, R., and Doxsey, S.J. (1999). Direct interaction of pericentrin with cytoplasmic dynein light intermediate chain contributes to mitotic spindle organization. Journal of Cell Biology 147, 481-491.

Qi, Y., Andolfi, L., Frattini, F., Mayer, F., Lazzarino, M., and Hu, J. (2015). Membrane stiffening by STOML3 facilitates mechanosensation in sensory neurons. Nature Communications 6, 8512 . 
Quallo, T., Vastani, N., Horridge, E., Gentry, C., Parra, A., Moss, S., Viana, F., Belmonte, C., Andersson, D.a., and Bevan, S. (2015). TRPM8 is a neuronal osmosensor that regulates eye blinking in mice. Nature Communications 6, 7150.

Ranade, S.S., Syeda, R., and Patapoutian, A. (2015). Mechanically activated ion channels. Neuron 87, 1162-1179.

Ranade, S.S., Woo, S.-H., Dubin, A.E., Moshourab, R.a., Wetzel, C., Petrus, M., Mathur, J., Bégay, V., Coste, B., Mainquist, J., et al. (2014). Piezo2 is the major transducer of mechanical forces for touch sensation in mice. Nature 516, 121-125.

Roudaut, Y., Lonigro, A., Coste, B., Hao, J., Delmas, P., and Crest, M. (2012). Touch sense: functional organization and molecular determinants of mechanosensitive receptors. Channels (Austin, Tex) 6, 234-245.

Schindelin, J., Rueden, C.T., Hiner, M.C., and Eliceiri, K.W. (2015). The ImageJ ecosystem: An open platform for biomedical image analysis. Molecular Reproduction and Development 82, 518-529.

Schrenk-Siemens, K., Wende, H., Prato, V., Song, K., Rostock, C., Loewer, A., Utikal, J., Lewin, G.R., Lechner, S.G., and Siemens, J. (2014). PIEZO2 is required for mechanotransduction in human stem cell-derived touch receptors. Nature neuroscience 18, 10-16.

Shen, D., Wang, X., and Xu, H. (2011). Pairing Phosphoinositides with Calcium lons in Endolysosomal Dynamics. Bioessays 33, 448-457.

Shenton, F., Bewick, G.S., and Banks, R.W. (2014). A study of the expression of small conductance calcium-activated potassium channels (SK1-3) in sensory endings of muscle spindles and lanceolate endings of hair follicles in the rat. PloS one 9, e107073.

Soattin, L., Fiore, M., Gavazzo, P., Viti, F., Facci, P., Raiteri, R., Difato, F., Pusch, M., and Vassalli, M. (2016). The biophysics of piezo1 and piezo2 mechanosensitive channels. Biophysical Chemistry 208.

Söderberg, O., Gullberg, M., Jarvius, M., Ridderstråle, K., Leuchowius, K.-J., Jarvius, J., Wester, K., Hydbring, P., Bahram, F., Larsson, L.-G., et al. (2006). Direct observation of individual endogenous protein complexes in situ by proximity ligation. Nature methods 3 , 995-1000.

Spitz, F., Gonzalez, F., and Duboule, D. (2003). A global control region defines a chromosomal regulatory landscape containing the HoxD cluster. Cell 113, 405-417.

Srivastava, S., Li, Z., Lin, L., Liu, G., Ko, K., Coetzee, W.A., and Skolnik, E.Y. (2005). The Phosphatidylinositol 3-Phosphate Phosphatase Myotubularin- Related Protein 6 ( MTMR6) Is a Negative Regulator of the Ca 22 -Activated K 2 Channel K Ca 3 . 1. Society 25, 36303638. 
Syeda, R., Xu, J., Dubin, A.E., Coste, B., Mathur, J., Huynh, T., Matzen, J., Lao, J., Tully, D.C., Engels, I.H., et al. (2015). Chemical activation of the mechanotransduction channel Piezo1. elife 4.

Tymanskyj, S.R., Yang, B., Falnikar, A., Lepore, A.C., and Ma, L. (2017). MAP7 Regulates Axon Collateral Branch Development in Dorsal Root Ganglion Neurons. The Journal of Neuroscience 37, 1648-1661.

Usoskin, D., Furlan, A., Islam, S., Abdo, H., Lönnerberg, P., Lou, D., Hjerling-Leffler, J., Haeggström, J., Kharchenko, O., Kharchenko, P.V., et al. (2015). Unbiased classification of sensory neuron types by large-scale single-cell RNA sequencing Dmitry. Nature neuroscience 18, 145-153.

Vaccari, I., Dina, G., Tronchere, H.I.n., Kaufman, E., Chicanne, G.t., Cerri, F., Wrabetz, L., Payrastre, B., Quattrini, A., Weisman, L.S., et al. (2011). Genetic interaction between MTMR2 and FIG4 phospholipid phosphatases involved in Charcot-Marie-Tooth neuropathies. PLoS Genetics 7.

Viatchenko-Karpinski, V., and Gu, J.G. (2016). Mechanical sensitivity and electrophysiological properties of acutely dissociated dorsal root ganglion neurons of rats. Neuroscience Letters 634, 70-75.

Vilceanu, D., and Stucky, C.L. (2010). TRPA1 mediates mechanical currents in the plasma membrane of mouse sensory neurons. PloS one 5, e12177.

Walsh, B.W., Lenhart, J.S., Schroeder, J.W., and Simmons, L.A. (2012). Far western blotting as a rapid and efficient method for detecting interactions between DNA replication and DNA repair proteins. Methods Mol Biol 922, 161-168.

Wetzel, C., Hu, J., Riethmacher, D., Benckendorff, A., Harder, L., Eilers, A., Moshourab, R., Kozlenkov, A., Labuz, D., Caspani, O., et al. (2007). A stomatin-domain protein essential for touch sensation in the mouse. Nature 445, 206-209.

Woo, S.-H., Lukacs, V., de Nooij, J.C., Zaytseva, D., Criddle, C.R., Francisco, A., Jessell, T.M., Wilkinson, K.a., and Patapoutian, A. (2015). Piezo2 is the principal mechanotransduction channel for proprioception. Nature Neuroscience, 1-8.

Woo, S.-H., Ranade, S., Weyer, A.D., Dubin, A.E., Baba, Y., Qiu, Z., Petrus, M., Miyamoto, T., Reddy, K., Lumpkin, E.a., et al. (2014). Piezo2 is required for Merkel-cell mechanotransduction. Nature 509, 622-626.

Wu, J., Goyal, R., Grandl, J., Coste, B., Coste, B., Coste, B., Ge, J., Li, J., Woo, S.H., Maksimovic, S., et al. (2016a). Localized force application reveals mechanically sensitive domains of Piezo1. Nature Communications 7.

Wu, J., Lewis, A.H., and Grandl, J. (2016b). Touch, Tension, and Transduction - The Function and Regulation of Piezo lon Channels. Trends in Biochemical Sciences 42. 
Xu, X.Z.S. (2016). Demystifying Mechanosensitive Piezo Ion Channels. Neuroscience Bulletin 32.

Yan, Z., Zhang, W., He, Y., Gorczyca, D., Xiang, Y., Cheng, L.E., Meltzer, S., Jan, L.Y., and Jan, Y.N. (2013). Drosophila NOMPC is a mechanotransduction channel subunit for gentletouch sensation. Nature 493.

Yu, H., Pan, B., Weyer, A., Wu, H.-E., Meng, J., Fischer, G., Vilceanu, D., Light, A.R., Stucky, C., Rice, F.L., et al. (2015). CaMKII Controls Whether Touch Is Painful. The Journal of Neuroscience 35 14086-14102.

Zhao, Q., Wu, K., Geng, J., Zhi, P., Zhang, M., Correspondence, B.X., Chi, S., Wang, Y., and Xiao, B. (2016). Ion Permeation and Mechanotransduction Mechanisms of Mechanosensitive Piezo Channels. Neuron, 1-16.

Zimmerman, W.C., Sillibourne, J., Rosa, J., and Doxsey, S.J. (2004). Mitosis-specific anchoring of gamma tubulin complexes by pericentrin controls spindle organization and mitotic entry. Molecular biology of the cell 15, 3642-3657.

Zolov, S.N., Bridges, D., Zhang, Y., Lee, W.-w., Riehle, E., Verma, R., and Lenk, G.M. (2012). In vivo, Pikfyve generates $\mathrm{PI}(3,5) \mathrm{P} 2$, which serves as both a signaling lipid and the major precursor for PI5P. Proceedings of the National Academy of Sciences 109, 17472 17477.

Zu, Y., Gong, Y., Wan, L., Lv, Y., Cui, S., Jin, X., Li, C., and Chen, X. (2015). Pericentrin Is Related to Abnormal $\beta$-Cell Insulin Secretion through F-Actin Regulation in Mice. Plos One 10, e0130458. 


\section{ACKNOWLEDGEMENTS}

To begin with I would like to thank Dr. Manuela Schmidt and Dr. David Gomez Varela, for having me in the lab and providing a stimulating and fulfilling environment to complete my thesis. Most of the techniques used in this thesis were actually taught to me by either Manuela or David, therefore I must credit them for almost the entirety of my scientific expertise.

I would also like to thank the members of the lab. Sergej Zeiter, our lab technician without who's expertise in preparing DRG cultures, this work would not have been possible; Julia Sondermann who joined the lab shortly before me and helped set up a lot of the techniques used in this work; Niklas Micheal and Meike Hutte, doctoral students in the lab, who provided valuable input for the project. I must also thank a former post doc in the lab Dr. Tom Rouwette, who along with Julia was involved in the initial stages of the project and provided the framework for me to carry out my work. I also thank MPI EM for hosting our lab and providing numerous resources which have been invaluable to this work.

I also extend my deepest gratitude to the IMPRS Neuroscience program as part of which I first arrived in Göttingen. Special thanks to Prof. Michael Hörner and Sandra Drube, for providing assistance and counselling whenever needed. I would also like to thank the members of my thesis committee, Prof. Luis Pardo and Prof. Martin Göpfert, who have provided great feedback during discussions and guided the project all along.

I would also like to acknowledge the funding sources that have supported this project: GGNB, IMPRS Neuroscience and DFG CRC 889, Göttingen.

A special thank you to all my friends in Göttingen, especially to the class of 2012-13 of the IMPRS program, for making this time in Göttingen so great. I have to thank my flatmates (and ex flatmate) Sindhuja Gowrisankaran, Shrutee Jakhanwal and Luis Ramos for all the great food, discussions and support! You guys made these years in Göttingen a lot more fun (:)

And finally I would like to thank the most important people in my life, my parents! Thank you papa for always believing in me and letting me chose my own path. Amma, thank you being there and supporting me even when others might have had their doubts. I owe everything I have achieved so far and everything I will achieve to you both. It was hard staying so far away from you, but I hope I can make you proud wherever I am! I love you both. A special thank you to my grandparents! I hope I made you proud. I also thank my extended family, aunts, uncles and all my cousins who are the closest I ever had to siblings! I love you all :)

I dedicate this work to all those who have had to leave their homes whether by their choice or not...

All you who sleep tonight

Far from the ones you love,

No hand to left or right

And emptiness above -

Know that you aren't alone

The whole world shares your tears,

Some for two nights or one,

And some for all their years.

-Vikram Seth 


\section{Supplementary tables}

Table 1: Details of plasmids used in this study

\begin{tabular}{|l|l|}
\hline plasmid name & source \\
\hline pCMVSport6-Piezo2-GST IRES GFP & Kind gift from Prof. Ardem Patapoutian \\
\hline pCMV6-Mtmr2-myc-DDK & Origene \\
\hline pCMV6-Mtmr2 C417S-myc-DDK & Self-generated \\
\hline pCMVSport6-Pericentrin & Self-generated \\
\hline pCMVSport6 & Kind gift from Prof. Ardem Patapoutian \\
\hline pCDNA-Piezo1-IRES-myc & Kind gift from Prof. Ardem Patapoutian \\
\hline pCDNA3.1-myc-His & Invitrogen \\
\hline pGEM-T easy-Kv 1.1-HA & Source not available \\
\hline pCDNA3-GST & Kind gift from Prof. Ardem Patapoutian \\
\hline
\end{tabular}

Table 2: Details of antibodies used in this study

\begin{tabular}{|l|l|l|l|}
\hline antibody & $\begin{array}{l}\text { source, catalogue } \\
\text { number }\end{array}$ & application & dilution \\
\hline Chicken anti-GFP & $\begin{array}{l}\text { Life Technologies, } \\
\text { A10262 }\end{array}$ & $\begin{array}{l}\text { Immunohistochemistry, } \\
\text { Immunocytochemistry }\end{array}$ & $1: 500$ \\
\hline Mouse anti-FLAG & Sigma Aldrich, F1804 & Immunoblotting & $1: 500$ \\
\hline Mouse anti-myc & Santa Cruz, sc-47694 & $\begin{array}{l}\text { Immunocytochemistry, } \\
\text { Immunoblotting }\end{array}$ & $\begin{array}{l}1: 100 \\
1: 100\end{array}$ \\
\hline Mouse anti-Tuj-488 & $\begin{array}{l}\text { HISS Diagnostics, A488- } \\
\text { 435L }\end{array}$ & Immunohistochemistry & $1: 500$ \\
\hline Mouse anti-S100 & Chemicon, MAB079-1 & Immunohistochemistry & $1: 100$ \\
\hline Rabbit anti-GST & Santa Cruz, sc-459 & $\begin{array}{l}\text { Immunocytochemistry, } \\
\text { Immunoblotting, }\end{array}$ & $\begin{array}{l}1: 500 \\
1: 100\end{array}$ \\
\hline Rabbit anti-mtmr2 & Biotechne, NBP1-33724 & Immunohistochemistry & $1: 500$ \\
\hline Rabbit anti-Pcnt & Abcam, ab4448 & $\begin{array}{l}\text { Immunohistochemistry, } \\
\text { Immunocytochemistry }\end{array}$ & $\begin{array}{l}1: 500 \\
1: 500\end{array}$ \\
\hline Rabbit anti-Pcnt & $\begin{array}{l}\text { Biolegend (Covance), } \\
923701\end{array}$ & $\begin{array}{l}\text { Immunohistochemistry, } \\
\text { Immunocytochemistry, } \\
\text { Immunoblotting }\end{array}$ & $\begin{array}{l}1: 750 \\
1: 750 \\
1: 1000\end{array}$ \\
\hline
\end{tabular}




\begin{tabular}{|l|l|l|l|}
\hline Rabbit anti-Piezo2 & $\begin{array}{l}\text { Novus Biologicals, } \\
\text { NBP1-785324SS }\end{array}$ & Immunohistochemistry & $1: 200$ \\
\hline Rabbit anti-Piezo2 & $\begin{array}{l}\text { Novus Biologicals, } \\
\text { NBP1-785338SS }\end{array}$ & Immunohistochemistry & $1: 200$ \\
\hline WGA-555 & $\begin{array}{l}\text { Thermo Fisher Scientific, } \\
\text { W32464 }\end{array}$ & Immunocytochemistry & $1: 200$ \\
\hline Alexa Fluor 488 & Life Technologies & $\begin{array}{l}\text { Immunocytochemistry, } \\
\text { Immunohistochemistry }\end{array}$ & $1: 250$ \\
\hline Alexa Fluor 564 & Life Technologies & $\begin{array}{l}\text { Immunocytochemistry, } \\
\text { Immunohistochemistry }\end{array}$ & $1: 250$ \\
\hline Alexa Fluor 555 & Life Technologies & $\begin{array}{l}\text { Immunocytochemistry, } \\
\text { Immunohistochemistry }\end{array}$ & $1: 250$ \\
\hline $\begin{array}{l}\text { Alexa Fluor 647 } \\
\text { Frobe anti-mouse } \\
\text { PLUS }\end{array}$ & $\begin{array}{l}\text { Life Technologies } \\
\text { Fragment Donkey }\end{array}$ & $\begin{array}{l}\text { Immunocytochemistry, } \\
\text { Immunohistochemistry }\end{array}$ & $1: 250$ \\
\hline $\begin{array}{l}\text { Alexa Fluor 680 } \\
\text { Mrobe anti-rabbit }\end{array}$ & Life Technologies & Immunoblotting & $1: 8000$ \\
\hline $\begin{array}{l}\text { IRDye } 800 C W \\
\text { Duolink insitu PLA }\end{array}$ & $\begin{array}{l}\text { Sigma-COR } \\
\text { Imoldrich }\end{array}$ & Immunoblotting & $1: 8000$ \\
\hline
\end{tabular}

Table 3: Details of siRNA used in this study

\begin{tabular}{|l|l|l|}
\hline siRNA & Company & Sequence \\
\hline $\begin{array}{l}\text { SiGENOME Mouse Pcnt (18541) } \\
\text { siRNA-SMARTpool }\end{array}$ & Thermo scientific & $\begin{array}{l}\text { CAACAGAGAGGAACUGAAU } \\
\text { GGUCCCAGCUUGAGUCUAU } \\
\text { GAGAAACUGUCCAUAAUCA } \\
\text { GAGAUUAGCCUCCAUGUUU }\end{array}$ \\
\hline $\begin{array}{l}\text { GeneSolution siRNA (Cat No. } \\
\text { 1027416) } \\
\text { Mm_Mtmr2 }\end{array}$ & Qiagen & $\begin{array}{l}\text { TGAGATTATTATGGCATAGTA } \\
\text { CTGTTACTCTACTTTATCTAA } \\
\text { GAGGCTTGTGTCAGATCCAAA } \\
\text { CAGCTGGAAGACTTTACTAAT }\end{array}$ \\
\hline
\end{tabular}




\begin{tabular}{|l|l|l|}
\hline $\begin{array}{l}\text { siGENOME Mouse Lnp (69605) } \\
\text { siRNA- SMARTpool }\end{array}$ & Dharmacon & $\begin{array}{l}\text { GAACAGGUACGCCCUCAUA } \\
\text { CAAGUCACCUGUUCUCAGA } \\
\text { CUGUUUACCUGUUUAUUG } \\
\text { UGGCAUGGCUUUGAAGGAA }\end{array}$ \\
\hline $\begin{array}{l}\text { siGENOME Mouse Map/d2 } \\
\text { (78283) siRNA- SMARTpool }\end{array}$ & Dharmacon & $\begin{array}{l}\text { GAGGAAAGGCUGCGCAUAG } \\
\text { GAGCCAAGCUUCAGUAUGA } \\
\text { CAACAUCAACCAUGAAUUU } \\
\text { GCGCAUGGCUGACAAGUAU }\end{array}$ \\
\hline $\begin{array}{l}\text { ON-TARGETplus Non-targeting } \\
\text { siRNA\#1 }\end{array}$ & Dharmacon & UGGUUUACAUGUCGACUAA \\
\hline $\begin{array}{l}\text { AllStars Negative Control siRNA } \\
\text { (Cat no. 1027280) }\end{array}$ & Qiagen & Not available \\
\hline
\end{tabular}

Table 4: Details of primers used in this study

\begin{tabular}{|l|l|}
\hline Primer name & Primer sequence (5'-3') \\
\hline GAPDH_forward & CAATGAATACGGCTACAGCAAC \\
\hline GAPDH_reverse & TTACTCCTTGGAGGCCATGT \\
\hline Actin_forward & GATCAAGATCATTGCTCCTCCTG \\
\hline Actin_reverse & CAGCTCAGTAACAGTCCGCC \\
\hline Piezo2_forward & AGGCAGCACATAGGATGGAT \\
\hline Piezo2_reverse & GCAGGGTCGCTTCAGTGTA \\
\hline Pericentrin_forward & CAAGGAGCACACCAGCAAGA \\
\hline Pericentrin_reverse & CGTCCACACACTACGCAAGACT \\
\hline Mtmr2_forward & TGTACCCCACCATTGAAGAAA \\
\hline Mtmr2_reverse & TAAGAGCCCCTGCAAGAATG \\
\hline Lunapark_forward & GTGCTCTGGATAGAATTGTTGAATAC \\
\hline Lunapark_reverse & TGCTGACATATGAGGGCGTA \\
\hline Map7d2_forward & TTTGGACACAGGGACCACTAC \\
\hline Map7d2_reverse & TCATGGTTGATGTTGAAAGTTTG \\
\hline Mtmr2 mutagenesis primer_forward & GTGGTACACTCCAGTGATGGATG \\
\hline Mtmr2 mutagenesis primer_reverse & CACAGACGTCTTCCCAGA \\
\hline Pericentrin EcorV primer & AGCGATATCATGGAAGACGAGCAGGAG \\
\hline Pericentrin Spe1 primer & GCAACTAGTTCACTGTTTAATCTTCTGGTGG \\
\hline
\end{tabular}


Table 5: Details of peptides used in this study

\begin{tabular}{|l|l|l|}
\hline Peptide name & Company & Peptide sequence \\
\hline Piezo2 peptide-FLAG tagged & GenScript & EWWRKILKYFWMSVVIDYKDDDDKQNN \\
\hline Mtmr2 peptide-myc tagged & GenScript & VASFRSRGRIPVLSEQKLISEEDLQNN \\
\hline $\begin{array}{l}\text { Piezo2 3Q peptide- FLAG } \\
\text { tagged }\end{array}$ & GenScript & EWWQQILQYFWMSVVIDYKDDDDKQNN \\
\hline Piezo2 X- FLAG tagged & GenScript & LWMIRNRRKYAMISSPDYKDDDDKQNN \\
\hline TRPA1 peptide & $\begin{array}{l}\text { Alamone } \\
\text { labs }\end{array}$ & NSTGIINETSDHSE \\
\hline TRPV1 peptide & $\begin{array}{l}\text { Alamone } \\
\text { labs }\end{array}$ & EDAEVFKDSMVPGEK \\
\hline
\end{tabular}

Table 6: Details of reagents used in this study

\begin{tabular}{|l|l|}
\hline Product & Company \\
\hline 10X PBS (Phosphate-buffered saline) pH 7.4 & Life Technologies \\
\hline Agarose & Roth \\
\hline Apilimod & Cayman chemicals \\
\hline ATP (adenosine 5'-triphosphate) magnesium salt & Sigma-Aldrich \\
\hline Bluejuice loading buffer 10X & Life Technologies \\
\hline BSA (Bovine serum albumin) & Sigma-Aldrich \\
\hline CaCl 2 & Roth \\
\hline Collagenase & Worthington \\
\hline Complete ULTRA protease inhibitor cocktail tablets & Roche \\
\hline CsCl & Sigma-Aldrich \\
\hline DDM (Dodecyl-ß-D-maltoside) & Roth \\
\hline DMEM (Dulbecco's modified eagle medium), GlutaMAX & Life Technologies \\
\hline DMEM (Dulbecco's modified eagle medium)/F-12, \\
GlutaMAX & Life Technologies \\
\hline DMSO (dimethyl sulfoxide) & \\
\hline Donkey serum & Sigma-Aldrich \\
\hline Dosium deoxycholate & Dianova \\
\hline DTT (1,4- Dithiol-dl-threit-lsg.) & Roth \\
\hline Duolink insitu detection reagents orange & Sigma-Aldrich \\
\hline Duolink insito probemaker plus & Sigma-Aldrich \\
\hline
\end{tabular}




\begin{tabular}{|c|c|}
\hline DTT (DI- Dithiothretol) & Sigma-Aldrich \\
\hline Dynabeads Protein $\mathrm{G}$ & Life Technologies \\
\hline EDTA (ethylenediaminetetraaacetic acid) & Roth \\
\hline EGTA (ethylene glycol tetraacetic acid) & Roth \\
\hline FBS (Fetal bovine serum) & Life Technologies \\
\hline FugeneHD transfection reagent & Promega \\
\hline Glucose & Roth \\
\hline Glutathione & Thermo Science \\
\hline Glycerol & Roth \\
\hline Goat serum & Dianova \\
\hline Growth factors (NGF, GDNF, BDNF, NT-3, NT-4) & R\&D Systems \\
\hline GTP (guanosine 3'-triphosphate) sodium salt & Sigma-Aldrich \\
\hline $\begin{array}{l}\text { HEPES (4-2-hydroxyethyl)-1-piperazineethanesulfonic } \\
\text { acid) }\end{array}$ & Life Technologies \\
\hline Horse serum & Life Technologies \\
\hline $\mathrm{KCl}$ & Roth \\
\hline Laminin & Life Technologies \\
\hline Library Efficiency DH5 $\alpha$ Competent Cells & ThermoFisher Scientific \\
\hline $\mathrm{MgCl} 2$ & Roth \\
\hline Milk powder & Roth \\
\hline $\mathrm{NaCl}$ & Roth \\
\hline $\mathrm{NaOH}$ & Roth \\
\hline $\begin{array}{l}\text { Nitrocellulose Pre-Cut Blotting Membranes, } 0.45 \mu \mathrm{m} \\
\text { pore size }\end{array}$ & Thermo Fisher Scientific \\
\hline Nocodazole & Sigma-Aldrich \\
\hline NucleoSpin RNA extraction kit & Macherey Nagel \\
\hline NuPAGE blotting kit & Life Technologies \\
\hline NuPAGE 3-8\% Tris-Acetate Protein Gels & Thermo Fisher Scientific \\
\hline OCT (optimal cutting temperature) compound & Sakura \\
\hline OneTaq One-Step RT-PCR Kit & New England Biolabs \\
\hline OPTIMEM medium & Life Technologies \\
\hline Papain & Worthington \\
\hline Penicillin/Streptomycin & Life Technologies \\
\hline PFA (paraformaldehyde) & Science services \\
\hline $\mathrm{PI}(3,5) \mathrm{P}_{2}$ & Echelon \\
\hline
\end{tabular}




\begin{tabular}{|l|l|}
\hline PI(3)P & Echelon \\
\hline Pierce Glutathione Magnetic beads & Thermo Science \\
\hline PIP Strips & Echelon \\
\hline Poly-D-Lysine & Milipore \\
\hline Power SYBR green PCR master mix & Life Technologies \\
\hline Q5 Site-directed mutagenesis kit & New England Biolabs \\
\hline QuantiTech Rev. Transcription Kit & Qiagen \\
\hline RPMI (Roswell Park Memorial Institute) medium & Life Technologies \\
\hline Slowfade Gold antifade reagent with DAPI & Life Technologies \\
\hline Sucrose & Merck \\
\hline Tris-acetate gel (3-8\%) & Thermo Fisher Scientific \\
\hline Tris-HCl & Roth \\
\hline Triton X-100 & Roth \\
\hline Tween 20 & Roth \\
\hline Wortmannin & Sigma-Aldrich \\
\hline
\end{tabular}


$\underline{\text { Table 7: List of abbreviations }}$

\begin{tabular}{|c|c|}
\hline AMPA & a-amino-3-hydroxy-5methyl-4isoxazolepropionic acid \\
\hline AMPAR & a-amino-3-hydroxy-5methyl-4isoxazolepropionic acid receptor \\
\hline ANOVA & Analysis of Variance \\
\hline $\mathrm{AU}$ & Arbitrary units \\
\hline BDNF & Brain-derived neurotrophic factor \\
\hline Camk11 & $\mathrm{Ca}^{2+} /$ calmodulin-dependent protein kinase type II \\
\hline CED & C-terminal extracellular domain \\
\hline CLSM & Confocal laser scanning microscopy \\
\hline CMT4B1 & Charcot-Marie-Tooth disease Type 4B1 \\
\hline CTL & C-terminal loop \\
\hline CTRL & Control \\
\hline $\mathrm{DEG} / \mathrm{ENaC}$ & Degenerin/epithelial sodium channel \\
\hline DIV & Days in vitro \\
\hline DNA & Deoxyribonucleic acid \\
\hline DRG & Dorsal root ganglion \\
\hline EM & Electron microscopy \\
\hline GAPDH & Glyceraldehyde-3-phosphate dehydrogenase \\
\hline GDNF & Glial cell line-derived neurotrophic factor \\
\hline GFP & Green fluorescent protein \\
\hline GO & Gene ontology \\
\hline GST & Glutathione S-transferase \\
\hline HEK & Human embryonic kidney cells \\
\hline HTMR & High threshold mechanoreceptor \\
\hline IA-MA & Intermediately adapting mechanically activated current \\
\hline IP & Immunoprecipitation \\
\hline IRES & Internal ribosome entry site \\
\hline $\mathrm{kDa}$ & Kilo Dalton \\
\hline LC-MS/MS & Liquid chromatography-mass-spectrometry \\
\hline LTMR & Low threshold mechanoreceptor \\
\hline MA & Mechanically activated \\
\hline MS & Mass spectrometry \\
\hline MscL & Mechanosensitive channel of large conductance \\
\hline MscS & Mechanosensitive channel of small conductance \\
\hline
\end{tabular}




\begin{tabular}{|c|c|}
\hline Mtmr & Myotubularin-related protein \\
\hline MTOC & Microtubule organizing complex \\
\hline $\mathrm{NCBI}$ & National Center for Biotechnology information \\
\hline NF-200 & Neurofilament 200 \\
\hline NGF & Nerve growth factor \\
\hline Nomp & No mechanoreceptor potential \\
\hline NT-3 & Neurotrophin-3 \\
\hline NT-4 & Neurotrophin-4 \\
\hline PC & Polycystin channel \\
\hline PCM & Pericentriolar material \\
\hline Pent & Pericentrin \\
\hline PCR & Polymerase chain reaction \\
\hline $\mathrm{PI}$ & Phosphatidylinositol \\
\hline $\mathrm{PI}(3,5) \mathrm{P}_{2}$ & Phosphatidylinositol 3,5-bisphosphate \\
\hline $\mathrm{Pl}(4,5) \mathrm{P}_{2}$ & Phosphatidylinositol 4,5-bisphosphate \\
\hline PI3P & Phosphatidylinositol 3-phosphate \\
\hline PIP & Phosphatidylinositol phosphate \\
\hline PLA & Proximity ligation assay \\
\hline RA-MA & Rapidly adapting mechanically activated current \\
\hline RNA & Ribonucleic acid \\
\hline SA-MA & Slowly adapting mechanically activated current \\
\hline SEM & Standard error of mean \\
\hline Stoml3 & Stomatin-like protein-3 \\
\hline TG & Trigeminal ganglion \\
\hline $\mathrm{TH}$ & Tyrosine hydroxylase \\
\hline TMC & Transmembrane channel \\
\hline TMHS & Tetra-span membrane protein of hair cell stereocilia \\
\hline TMIE & Transmembrane inner ear expressed gene \\
\hline TRP & Transient receptor potential \\
\hline VGLUT & Vesicular glutamate transport \\
\hline
\end{tabular}




\section{DECLARATION}

Herewith I declare, that I prepared the dissertation "Touching upon regulators of Piezo2 in mouse somatosensation" on my own and with no other sources and aids than quoted.

Göttingen,

September 2017

Pratibha Narayanan 


\section{CURRICULUM VITAE}

\section{PERSONAL INFORMATION}

Name: Pratibha Narayanan

Date and place of birth: 12 February 1991, New Delhi, India

Nationality: Indian

Office address: Max Planck Institute for Experimental Medicine

Hermann-Rein-Strasse. 3

D-37075, Göttingen

Phone: $+49(0) 551$ 3899-575

\section{EDUCATION}

2014- $\quad$ PhD student, Somatosensory Signaling and Systems Biology Group

Present

Max Planck Institute of Experimental Medicine, Göttingen, Germany

Supervisor: Dr. Manuela Schmidt

Thesis: Touching upon regulators of Piezo2 in mouse somatosensation

2012-2014 MSc student, IMPRS Neuroscience

European Neuroscience Institute, Göttingen, Germany

Supervisor: Dr. Ira Milosevic

Thesis: Molecular basis of epilepsy in endophilin knockout mice

2009-2012 BSc student

Sri Venkateswara College, Delhi University, New Delhi, India

\section{STIPENDS}

- $\quad$ GGNB Travel grant for Society for Neuroscience conference, 2016

- Stipend of the Excellence Foundation for the promotion of the Max Planck Society (2012-13) 


\section{CONFERENCE PARTICIPATION}

2016

2016

2016

2015
Society for Neuroscience (SFN) conference

Oral presentation: Identification and characterization of native Piezo2 interactors Federation of European Neuroscience Societies (FENS) conference

Poster presentation: Identification and characterization of Piezo2 interactors Neurizons

Poster presentation: Identification and characterization of Piezo2 interactors Neurizons

Poster presentation: Insights into vertebrate mechanotransduction-Characterizing interactors of the mechanosensitive ion channel, Piezo2

\section{PUBLICATIONS}

- Mtmr2 and PI(3,5)P2 interdependently control Piezo2-mediated mechanotransduction in peripheral sensory neurons

Narayanan P, Hütte M, Kudryasheva G, Francisco T, Lechner S, Rehlfelft F, Gomez-Varela D, Schmidt M. eLife 2018;7:e32346 doi: 10.7554/eLife.32346.

- Native Piezo2 Interactomics Identifies Pericentrin as a Novel Regulator of Piezo2 in Somatosensory Neurons.

Narayanan P, Sondermann J, Rouwette T, Karaca S, Urlaub H, Mitkovski M, Gomez-Varela D, Schmidt M. Journal of Proteome Research. 2016 Aug 5;15(8):2676-87. doi: 10.1021/acs.jproteome.6b00235.

- Nocistatin sensitizes TRPA1 chennels in peripheral sensory neurons.

Avenali L, Abate Fulas O, Sondermann J, Narayanan P, Gomez-Varela D, Schmidt M. Channels (Austin). 2016 Jun 30:1-9.

- Alternatively Spliced Isoforms of KV10.1 Potassium Channels Modulate Channel Properties and Can Activate Cyclin-dependent Kinase in Xenopus Oocytes.

Ramos Gomes F, Romaniello V, Sánchez A, Weber C, Narayanan P, Psol M, Pardo LA. J Biol Chem. 2015 Dec 18;290(51):30351-65. doi: 10.1074/jbc.M115.668749. Epub 2015 Oct 30 .

- Modulation of nociceptive ion channels and receptors via protein-protein interactions: implications for pain relief.

Rouwette T, Avenali L, Sondermann J, Narayanan P, Gomez-Varela D, Schmidt M. Channels (Austin). 2015;9(4):175-85. doi: 10.1080/19336950.2015.1051270. Epub 2015 Jun 3.

- Annexin A2 regulates TRPA1-dependent nociception.

Avenali L, Narayanan P, Rouwette T, Cervellini I, Sereda M, Gomez-Varela D, Schmidt M. J Neurosci. 2014 Oct 29;34(44):14506-16. doi: 10.1523/JNEUROSCI.1801-14.2014 

